Florida International University

FIU Digital Commons

$10-9-2018$

\title{
Brain Connectivity Networks for the Study of Nonlinear Dynamics and Phase Synchrony in Epilepsy
}

Hoda Rajaei

Florida International University, hraja001@fiu.edu

Follow this and additional works at: https://digitalcommons.fiu.edu/etd

Part of the Bioelectrical and Neuroengineering Commons, Biomedical Commons, Computational Engineering Commons, and the Signal Processing Commons

\section{Recommended Citation}

Rajaei, Hoda, "Brain Connectivity Networks for the Study of Nonlinear Dynamics and Phase Synchrony in Epilepsy" (2018). FIU Electronic Theses and Dissertations. 3882.

https://digitalcommons.fiu.edu/etd/3882

This work is brought to you for free and open access by the University Graduate School at FIU Digital Commons. It has been accepted for inclusion in FIU Electronic Theses and Dissertations by an authorized administrator of FIU Digital Commons. For more information, please contact dcc@fiu.edu. 


\section{FLORIDA INTERNATIONAL UNIVERSITY}

Miami, Florida

\section{BRAIN CONNECTIVITY NETWORKS FOR THE STUDY OF NONLINEAR DYNAMICS AND PHASE SYNCHRONY IN EPILEPSY}

A dissertation submitted in partial fulfillment of

the requirements for the degree of

DOCTOR OF PHILOSOPHY

in

ELECTRICAL ENGINEERING

by

Hoda Rajaei

2018 
To: Dean John Volakis

College of Engineering and Computing

This dissertation, written by Hoda Rajaei, and entitled Brain Connectivity Networks for the Study of Nonlinear Dynamics and Phase Synchrony in Epilepsy, having been approved in respect to style and intellectual content, is referred to you for judgment.

We have read this dissertation and recommend that it be approved.

Armando Barreto

Jean Andrian

Naphtali Rishe

Malek Adjouadi

Mercedes Cabrerizo, Major Professor

Date of Defense: November 9,2018

The dissertation of Hoda Rajaei is approved.

Dean John Volakis

College of Engineering and Computing

Andrés G. Gil

Vice President for Research and Economic Development and Dean of the University Graduate School

Florida International University, 2018 
(C) Copyright 2018 by Hoda Rajaei

All rights reserved. 


\section{DEDICATION}

I want to dedicate this dissertation:

To my dear husband, Mohammad, for his love, support, encouragement, and his invaluable help. This long journey wouldn't be possible without having him by my side.

To my little son, Arvin who is a new hope and motivation in our life. His birth opened a new window to the beauties of life for us.

To my dear parents Mrs. Farzaneh Amirzadeh and Mr. Abdolreza Rajaei, for their love and support that helped me in this journey.

To my kind sister Dr. Mona Rajaei for her help and sharing her clinical knowledge that lightens my view for my work.

To my brother and precious family - Mostafa, my lovely little Diana and Rasa, my in-laws, especially Mr. Kazem Moravej and Ms. Atiyeh Haghdan, for their continuous support. 


\section{ACKNOWLEDGMENTS}

I would like to express my sincere gratitude and appreciation to my major advisor Dr. Mercedes Cabrerizo and Dr. Malek Adjouadi for their valuable and priceless mentorship, advice and guidance through all the steps of my doctoral studies and research.

Furthermore, I would like to thank my dissertation committee members Dr. Armado Barreto, Dr. Jean Andrian, and Dr. Naphtali Rishe for their help, valuable input, and accessibility.

I would like to thank my friends Ms. Shunfei Li and Mr. Saman Sargolzaei for their precious help during my study.

The financial support provided by National Science Foundation under grant numbers: CNS 1532061, CNS-1551221, CNS-1429345 and CNS-1338922. Support of the Ware Foundation is also greatly appreciated. I should acknowledge the university graduate school for offering me the Dissertation Year Fellowship (DYF) to support the writing stage of my dissertation. 


\title{
ABSTRACT OF THE DISSERTATION
}

\section{BRAIN CONNECTIVITY NETWORKS FOR THE STUDY OF NONLINEAR DYNAMICS AND PHASE SYNCHRONY IN EPILEPSY}

\author{
by
}

\section{Hoda Rajaei}

Florida International University, 2018

\author{
Miami, Florida \\ Professor Mercedes Cabrerizo, Major Professor
}

\begin{abstract}
Assessing complex brain activity as a function of the type of epilepsy and in the context of the 3D source of seizure onset remains a critical and challenging endeavor. In this dissertation, we tried to extract the attributes of the epileptic brain by looking at the modular interactions from scalp electroencephalography (EEG). A classification algorithm is proposed for the connectivity-based separation of interictal epileptic EEG from normal. Connectivity patterns of interictal epileptic discharges were investigated in different types of epilepsy, and the relation between patterns and the epileptogenic zone are also explored in focal epilepsy.
\end{abstract}

A nonlinear recurrence-based method is applied to scalp EEG recordings to obtain connectivity maps using phase synchronization attributes. The pairwise connectivity measure is obtained from time domain data without any conversion to the frequency 
domain. The phase coupling value, which indicates the broadband interdependence of input data, is utilized for the graph theory interpretation of local and global assessment of connectivity activities.

The method is applied to the population of pediatric individuals to delineate the epileptic cases from normal controls. A probabilistic approach proved a significant difference between the two groups by successfully separating the individuals with an accuracy of 92.8\%. The investigation of connectivity patterns of the interictal epileptic discharges (IED), which were originated from focal and generalized seizures, was resulted in a significant difference $(P-$ value $\ll 0.01)$ in connectivity matrices. It was observed that the functional connectivity maps of focal IED showed local activities while generalized cases showed global activated areas. The investigation of connectivity maps that resulted from temporal lobe epilepsy individuals has shown the temporal and frontal areas as the most affected regions.

In general, functional connectivity measures are considered higher order attributes that helped the delineation of epileptic individuals in the classification process. The functional connectivity patterns of interictal activities can hence serve as indicators of the seizure type and also specify the irritated regions in focal epilepsy. These findings can indeed enhance the diagnosis process in context to the type of epilepsy and effects of relative location of the 3D source of seizure onset on other brain areas. 
CHAPTER

Chapter 1

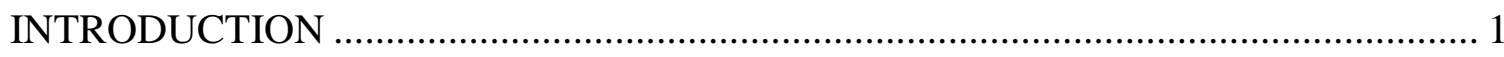

1.1 An Introduction to Epilepsy as a Neurological Disorder ................................ 1

$1.2 \quad$ General Statement of Problem Area .......................................................... 3

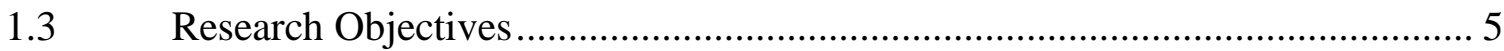

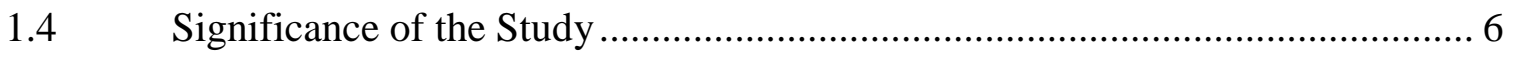

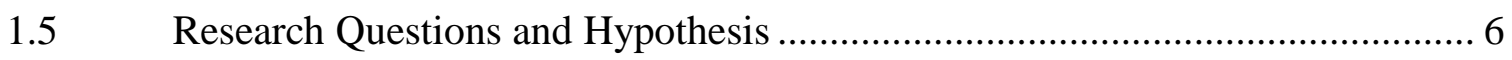

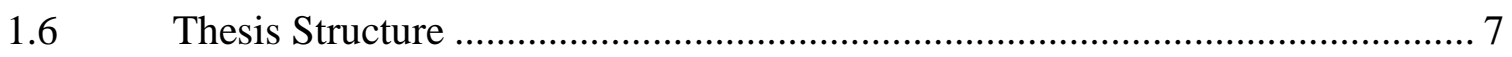

Chapter 2

LITERATURE REVIEW AND DESIGN FUNDAMENTALS .................................. 10

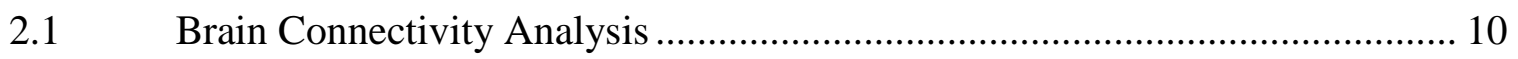

2.2 A brief review of functional connectivity measures ................................... 11

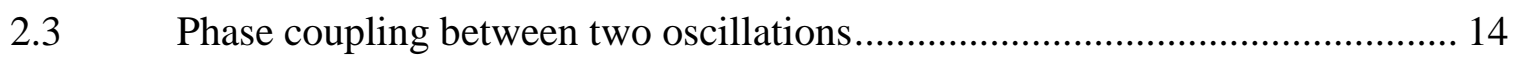

$2.4 \quad$ Nonlinear methods for phase synchrony analysis.................................... 16

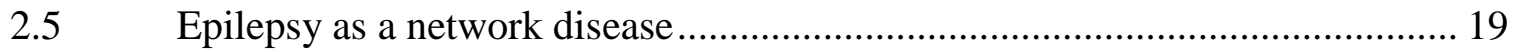

Chapter 3

NONLINEAR ESTIMATION OF THE BRAIN FUNCTIONAL CONNECTIVITY .... 23

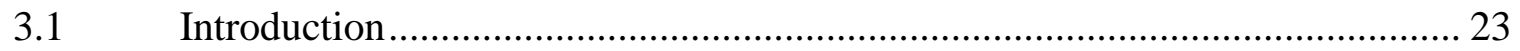

3.2 Functional Connectivity based on the Probability of Recurrence ................... 24

3.3 Parameter Selection and Significance Evaluation ....................................... 27

3.4 Brain Network Interpretation Using Graph Theory .................................... 29

Chapter 4

CLUSTERING BY FUNCTIONAL CONNECTIVITY ......................................... 31

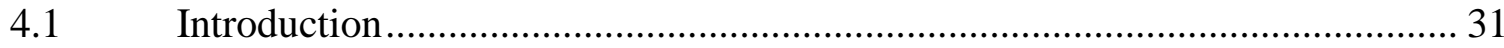

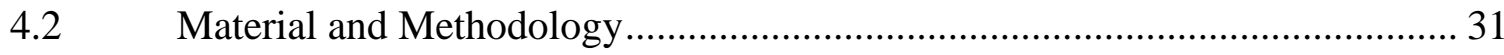

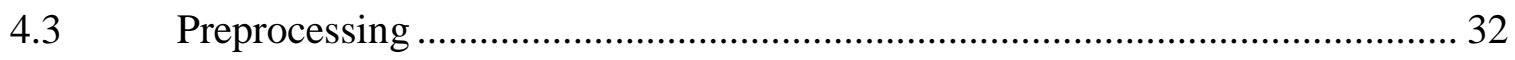

viii 
4.4 Functional Connectivity and Feature Extraction ........................................ 35

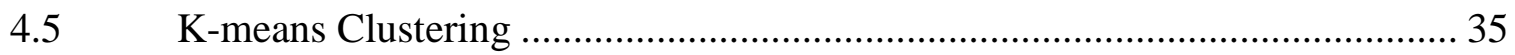

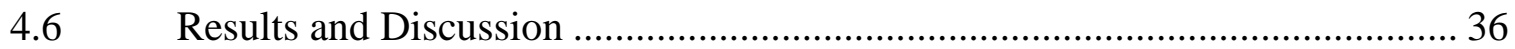

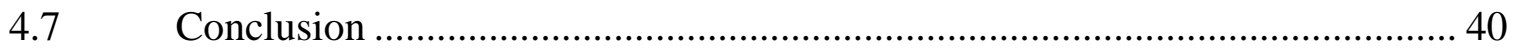

Chapter 5

CONNECTIVITY MAPS OF DIFFERENT TYPES OF EPILEPTOGENIC

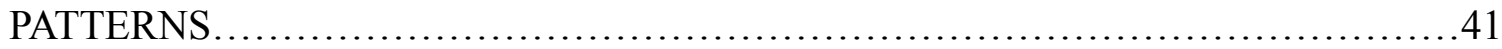

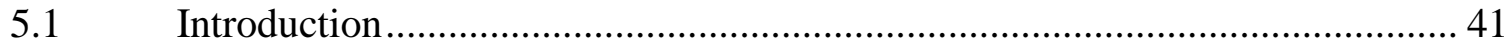

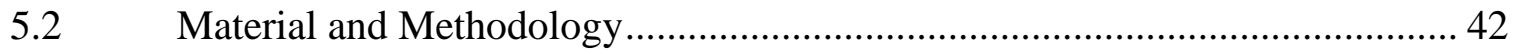

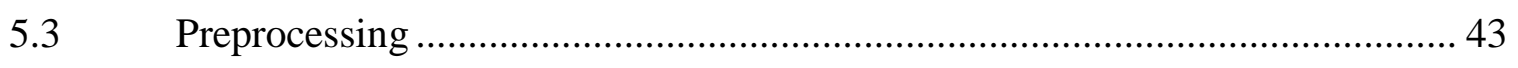

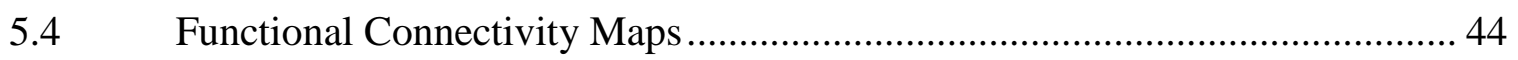

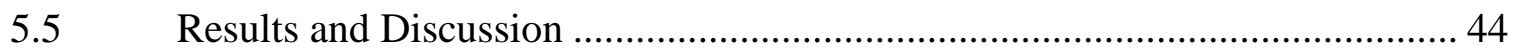

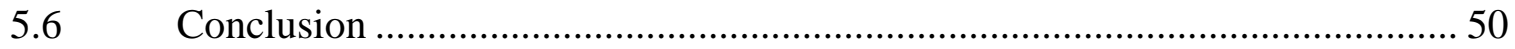

Chapter 6

CONNECTIVITY DYNAMICS OF INTERICTAL EPILEPTIFORM ACTIVITY ....... 52

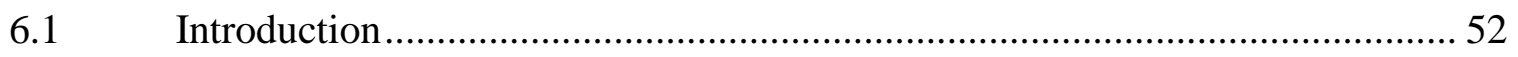

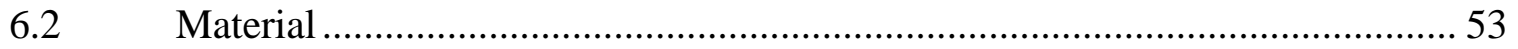

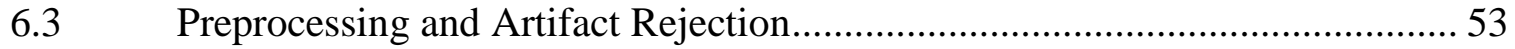

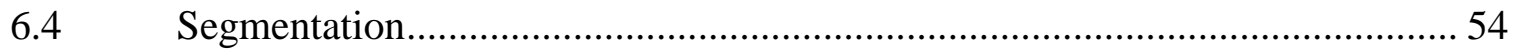

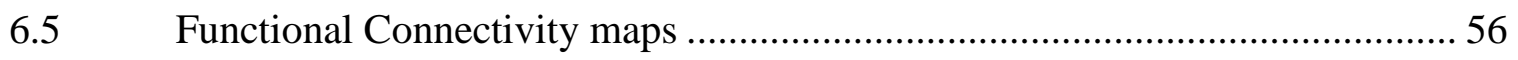

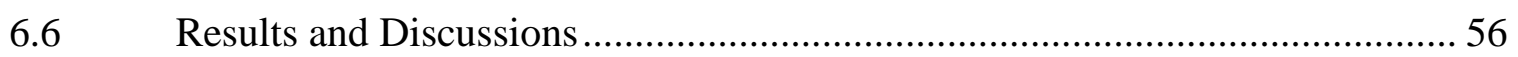

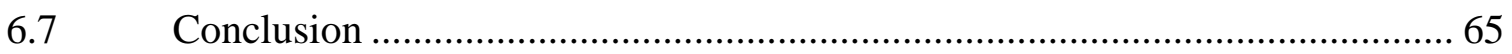

Chapter 7

DYNAMICS AND DISTANT EFFECTS OF TEMPORAL EPILEPTOGENIC FOCUS USING FUNCTIONAL CONNECTIVITY MAPS ................................................. 67

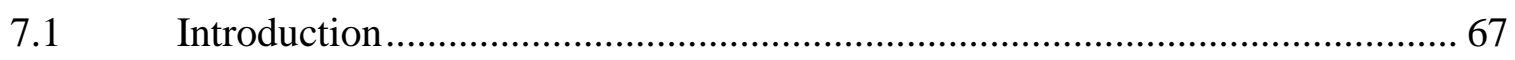

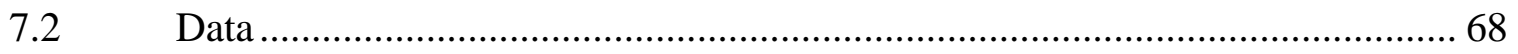

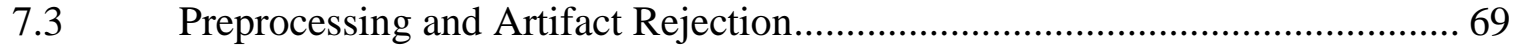

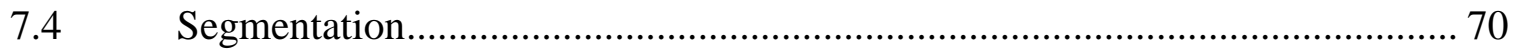


7.5 Functional Connectivity Maps and Parameters ........................................ 71

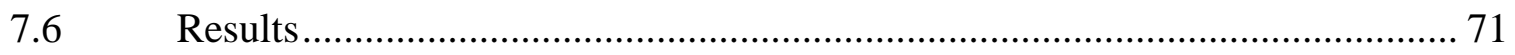

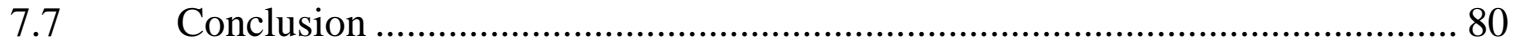

Chapter 8

CONCLUSION AND RECOMMENDATIONS FOR FUTURE WORK ...................... 82

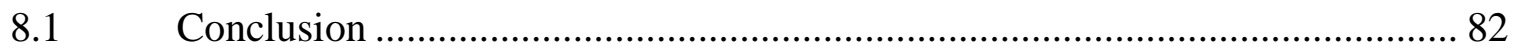

8.2 Development and future work recommendation.......................................... 86

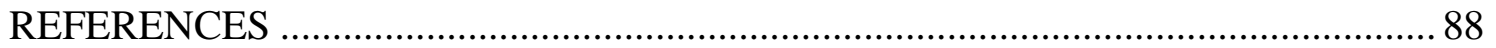

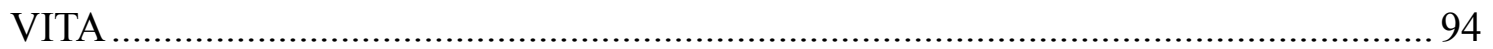




\section{LIST OF TABLES}

TABLE

PAGE

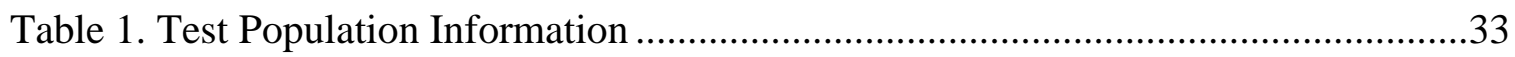

Table 2: Brief Description of Extracted Features ......................................................

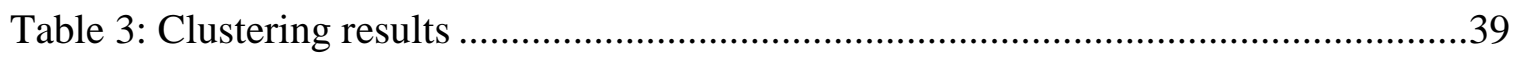

Table 4: Demographic Information of Participants .................................................53

Table 5. The Mean and standard deviation of average spike groups ..............................57

Table 6: Demographic information of participants....................................................69

Table 7: Six brain local regions and related electrodes ................................................74 


\section{LIST OF FIGURES}

FIGURE

PAGE

Figure 1. The definition of phase synchronization for two signals (A) Complete synchronization with zero phase difference in a specific frequency (B) Phase synchrony with phase difference of $\pi / 2$ in a specific frequency(C) State of phase synchrony among two signal with variable phase.

Figure 2: Flowchart for the suggested algorithm....

Figure 3: Connectivity head map of average connectivity matrices for controls and epileptic patients (a) higher $30 \%$ connections and (b) higher $50 \%$ connections

Figure 4: Number of strong connections based on different thresholds for average connectivity matrices

Figure 5: Sample of three epileptiform patterns in one electrode segment: (a) Single spike, (b) Complex spike (c) Repetitive spike

Figure 6: The block diagram of the connectivity matrices evaluation.

Figure 7: Histogram diagrams of a sample connectivity matrix of each spike group.

Figure 8: Connectivity maps from average connectivity matrices of the three spike groups.

Figure 9: The quantification comparison for the main brain regions within three epileptic patterns.

Figure 10: The Q-Q plot of the residuals of the ANOVA test confirms the normality of the study data.

Figure 11: Multi-comparison statistic test results. The single spike group has no overlap with the complex or repetitive group indicating a significant difference with respect to the other two groups.

Figure 12: Samples of EEG segments used for connectivity analysis. (a) Spike (b) Spike-wave complexes (Complex spike) (c) Poly spike-wave complexes (Repetitive spike)

Figure 13: Histogram of average EEG connectivity maps. (a) Spike (b) Complex spike

(c) Repetitive spike 
Figure 14: The comparison of synchronization value among three spike categories shows synchronization in spikes are lower than complex and repetitive spikes.

Figure 15: Multi Comparison test results shows that average connections of spikes are significantly different from complex and repetitive spikes

Figure 16: The clustering coefficient (top), shortest path length (down) of the EEG connectivity maps for the three spike categories.

Figure 17: The small world index which shows that the epileptic interictal activities change the connectivity graph topology toward a random graph.

Figure 18: Head map connectivity plots of various epileptiform discharges extracted from EEG segments containing: a) Single spike b) Complex spike c) Repetitive spike...63

Figure 19: The quantification of average connectivity matrices in four main brain regions (anterior/posterior regions and left/right hemispheres). Each graph shows the number of connections (vertical axis) in various threshold levels (Horizontal axis). .......65

Figure 20: Sample segments of data: (a) Spike (b) Spike followed by a wave and (c)

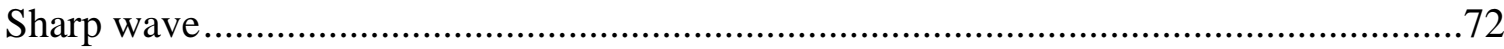

Figure 21: The different local brain regions considered: ..........................................73

Figure 22. Head map connectivity plot of six local regions resulting from a sample EEG segment of (a) P3 and (b) P4 with epileptic focus in right temporal and (c) P1 and (d) P9 with an epileptic focus in left temporal area. Note: only strong connections are shown.

Figure 23. Head map connectivity plot of six local regions yielded from a sample EEG segment of (a) P3 and (b) P4 with epileptic focus in right temporal and (c) P1 and (d) P9 with an epileptic focus in left temporal area. Note: Only strong connections are shown.

Figure 24. Average activity percentage of local regions for the 10 patients in the study..78

Figure 25. Average activity percentage of local and distant regions for the 10 patients in the study 


\section{SYMBOLS AND ABBREVIATIONS}

\begin{tabular}{|c|c|}
\hline CPR & Correlation based on Probability of Recurrence \\
\hline DTI & Diffusion Tensor Imaging \\
\hline ECG & Electrocardiography \\
\hline EEG & Electroencephalography \\
\hline EMG & Electromyography \\
\hline $\mathrm{EZ}$ & Epileptogenic Zone \\
\hline fMRI & functional Magnetic Resonance Imaging \\
\hline iAAFT & iterative Amplitude Adjusted Fourier Transform \\
\hline IED & Interictal Epileptiform Discharge \\
\hline LFP & Local Field Potential \\
\hline LTLE & Left Temporal Lobe Epilepsy \\
\hline MEG & Magnetoencephalography \\
\hline MRI & Magnetic Resonance Imaging \\
\hline PET & Positron Emission Tomography \\
\hline PS & Phase Synchronization \\
\hline $\mathrm{RP}$ & Recurrence Plot \\
\hline RQA & Recurrence Quantification Analysis \\
\hline RTLE & Right Temporal Lobe Epilepsy \\
\hline SNR & Signal to Noise Ratio \\
\hline TLE & Temporal Lobe Epilepsy \\
\hline WHO & World Health Organization \\
\hline
\end{tabular}




\section{Chapter 1}

\section{INTRODUCTION}

\subsection{An Introduction to Epilepsy as a Neurological Disorder}

Despite the significant recent advances in the field of neuroscience, human brain function in its healthy state and changes that occur due to a given neurological dysfunction are complex neuronal choreographies that are yet to be understood. The brain as a center for emotions, behavior and cognitive responses of the human being requires thorough investigations of brain activity and causal effect in both its normal and abnormal states through high temporal and spatial resolution as can be observed through electroencephalograms (EEG) and neuroimaging technologies. In spite of the many advances made in the study of neurological disorders, there are so many unknown aspects of their etiology or treatment methods (Sadock et al., 2015).The most serious limitation about the research of the human brain's functionality is the simple fact that there is no direct way to examine it in its "working state." Almost all pertinent information we have gathered thus far has been through studying the brain of animal species or cadavers with the assumption that such findings can partially be extended to the living human brain (Sadock et al., 2015).

However, modern neuroimaging techniques such as functional magnetic resonance imaging (fMRI), positron emission tomography (PET), Diffusion Tensor Imaging (DTI), Computed Tomography and Magnetoencephalography (MEG) in concert with temporal data offered through EEG recordings along with the help of advanced computational 
neuroscience algorithms has helped the research community to determine ways to improve the monitoring process, enhance the prospects for accurate diagnosis and plan for early intervention and treatment protocols. Such advances have made it possible to carry out more precise assessments of brain activity through structural, functional and physiological measurements recorded through newer recording devices and with advanced technological breakthroughs. Any new developments to increase our knowledge of the human brain will be based on the progress in many disciplines, from medicine and neurobiology to neural science, physics, engineering, and computing.

As proclaimed by the World Health Organization (WHO), epilepsy is the fourth most common neurological disorder, which affects more than 50 million people with the rate of 2.4 million new cases each year throughout the world (http://www.who.int/newsroom/fact-sheets/detail/epilepsy). The disease affects all races, genders and age groups, from children to elderly people. In the United States, about $1.8 \%$ of adults, 18 years and older, and $1 \%$ of children, aged 0-17, are reported as epileptic by the Center for Disease Control and Prevention (Centers for Disease Control and Prevention, (CDC), 2012). Epilepsy can happen to anybody and at any age and one's lifetime. In fact, the probability of one experiencing an episode of seizure is estimated to be about $8 \%$ to $10 \%$ and for the risk of actually having epilepsy would be about 3\% (Pohlmann-Eden et al., 2006). Although epilepsy could appear at any age, the disease has a tendency to occur during childhood and adolescence. It is estimated that around 50\% of epileptic cases are initiated in childhood (Acharya et al., 2013). This makes the study of pediatric epilepsy, an essential 
part of the disease research since the early treatment will highly improve the patient's quality of life.

Epilepsy or epileptic seizure disorder is identified by the recurrent brain excessive synchronous electrical activity, which is called seizure (Acharya et al., 2013). Seizure is a paroxysmal, sudden electrical discharge of the brain neurons which can affect one region of the cortex or it may start in one region and extend to other parts to affect the brain globally. The unpredictability of the seizure causes the patient to suffer from secondary conditions and injuries that impact the patients' normal life. Epilepsy is recognized as two major types, focal and generalized. In focal or partial epilepsy, seizures are initiated from a specific part of the brain, known as the epileptic foci. Epilepsy can thus engage only a small part of the brain or extends and affects the rest of the brain (secondary generalized epilepsy). In generalized epilepsy, seizure affects the whole brain.

\subsection{General Statement of Problem Area}

Epilepsy is a complex disease with many unclear and undefined phases. Since having seizures does not always result in an epilepsy diagnosis, epilepsy detection has turned into a time-consuming and expensive process of getting the patient's history and investigating brain activities using different modalities, mostly the EEG recordings. Knowing the particular disease's features will help physicians to prescribe the appropriate treatment for patients. For many years, scalp EEG has been used as a tool to diagnose and treat the disorder, and a myriad of studies, all with different practical and theoretical merits have 
been reported on this recording modality. Scalp EEG recording is simple and inexpensive compared to other forms of brain activity recordings. Also, it is a noninvasive modality which carries a vast amount of information about the disease (Tatum, 2014). The majority of epilepsy diagnosis is done by recording and visual observation of EEG during seizure and monitoring spikes in long interictal recordings. The unpredictability of seizures causes most EEG recordings to be in interictal phases, therefore, extracting the epileptic characteristics of EEG in interictal periods plays a key role in disease detection. There is evidence that EEG recordings of a normal non-epileptic person present different patterns from the EEG of an epileptic patient even without seizures. Consequently, extracting the hidden epileptic attributes of EEG in the interictal phase may be a beneficial tool to overcome the long and complex process of a correct diagnosis.

The knowledge of how the brain functions is still very limited. The brain consists of neurons, which are interacting cells performing complex sensorimotor and cognitive tasks through stimulation or inhibition. A task is completed by neurons grouped in functional modules communicating together and forming a functional connectivity network. The study of brain functional connectivity networks would be of great import when a person is diagnosed with some neurological abnormalities. Functional connectivity maps are calculated using several modalities, such as fMRI, PET, MEG, and EEG, and different methods are used to extract features of brain connectivity that define the brain disorder. The study of interactions among cortical regions reveals useful information about the disease characteristics. The functional connectivity network of an epileptic brain would be a representation of the brain activity during the seizure and in preictal and interictal phases. 
It is hypothesized that an epileptic brain is more connected compared to the normal brain (Rangaprakash and Pradhan, 2014). Thus connectivity maps of interictal data may be used to differentiate between epileptic and control brains (Rajaei et al., 2015). The investigation of connectivity maps of epileptic biomarkers may help identify the characteristics of the disease in various types of focal or generalized epilepsy diagnoses. The analysis of interictal EEG data may be utilized in finding the epileptogenic foci more precisely.

\subsection{Research Objectives}

The analysis of various brain modalities to extract the hidden features of neurological disorders improves the understanding of the disease and would result in the enhancement of disease diagnosis and treatment. The connectivity analysis of the epileptic brain using multichannel scalp EEG, which is a noninvasive and inexpensive modality, is a prospective research area to fulfill the goal. This research aims to explore the connectivity features of the epileptic functional brain networks from interictal EEG data and using it to differentiate the epileptic patients from healthy control. The connectivity characteristics of epileptic biomarkers will be investigated to enhance the better understanding of the disease in between seizure stages. The connectivity maps will improve the recognition of the epileptogenic focus. 


\subsection{Significance of the Study}

The analysis of functional connectivity maps of epileptic patients is a method that can shed some new light on complex neurological diseases, such as epilepsy. This study outlines a reliable methodology that overcomes the obstacle of subjective epilepsy diagnosis. The significance of the study is in the application of a methodology that determines brain activity patterns that allows not only to identify the type of epilepsy a patient is experiencing (focal or generalized) without the inclusion of the ictal state itself, and in investigating how such activity patterns change in the way they affect distant areas of the brain in relation to the epileptogenic zone and thus the affected area of the brain which is thought to induce the occurrence of seizures.

\subsection{Research Questions and Hypothesis}

Question 1: $\quad$ How are the functional interactions of different brain regions alter due to epilepsy?

Hypothesis 1: An Epileptic brain is hypothesized to be abnormally highly connected in comparison with a normal brain. Therefore, an epileptic functional connectivity map is perceived to show more and stronger connections during certain events. As a consequence, the connectivity networks of these two groups are supposed to be statistically different.

Question 2: What would be the effect of different epileptic biomarkers in 
connectivity maps?

Hypothesis 2: It is expected that spikes, as epileptic biomarkers, will result in higher connections. Considering the various types of spikes related to focal and generalized epilepsy, diverse patterns are perceived based on the disease type.

Question 3: Do the connectivity maps of epileptic biomarkers relate to the epileptogenic focus?

Hypothesis3: Analysis of connectivity maps of interictal spikes shows more activation in some spikes. It is expected that source localization analysis of these spikes addresses the location of the epileptogenic source more accurately.

\subsection{Thesis Structure}

Chapter 1 provides an introduction to the research problem and defines the research objectives we seek, highlights what the significance of the study is, and enumerates the research questions we pose and the hypotheses that were tested.

Chapter 2 provides a review of the connectivity methods that have been reported in the literature. It provides a retrospective on what constitutes brain connectivity, provides a brief review on functional connectivity measures used, describes and defines the concepts of phase coupling between oscillations, and the nonlinear methods that are used for phase synchrony analysis. 
In Chapter 3, the more current methods that are applied to this research domain are reviewed and discussed. The focus in Chapter 3 is placed on the nonlinear estimation of the brain functional connectivity, emphasizing phase synchronization and the probability of recurrence.

Chapter 4, presents a novel algorithm that uses the interictal connectivity features for epileptic clustering. Chapter 4 introduces a new method that explores the use probability of recurrence and the correlation between electrodes in extracting phase synchronization and the functional connectivity maps of the brain from interictal EEG data recordings in order to optimize the classification of healthy controls from patients with epilepsy.

Chapter 5 investigates the connectivity patterns of interictal epileptic biomarkers. In this chapter, we show how through the 10-20 EEG recording system, a nonlinear data-driven method was deployed to extract connectivity matrices that helped identify network synchronization based on the number of connections considering all brain regions. These connectivity matrices were then used in a quantification process to assess the three types of spike patterns considered in relation to the type of seizure, focal or generalized.

Chapter 6 develops an investigation on the patterns of interictal activities that best characterize EEG events defined as sharp waves, spike, spike-wave complexes and polyspike-wave complexes in both focal and generalized epilepsy. The significance of the connectivity matrices was assured with the help of surrogate data testing methods. The contribution of this chapter relates to the development of a new method for finding optimal parameters or features for the classification process. 
Chapter 7 focuses on relating the results of the functional connectivity maps to the epileptogenic zone in focal epilepsy. The intent here is to see how a 3D source or epileptogenic zone thought to be the cause of these seizures affect brain activity near and around this suspected zone. If results are reliable based on EEG records and past patient evaluation, such findings could serve as a means to validate the determination of the 3D source through inverse solutions.

Chapter 8 provides a retrospective on the key developments of this dissertation and assesses the different results obtained using the different methods that were implemented throughout this dissertation. It also provides suggestions for future research endeavors on the basis of the empirical results obtained and the limitations we still confront in ascertaining the different and complex patterns as revealed through brain connectivity patterns. This last assertion is due mainly to the fact that although the high temporal resolution is attained through EEG recordings, the complex brain connectivity patterns could not be appropriately described with the spatial resolution offered through the 10-20 EEG montage commonly used for EEG analysis. 


\section{Chapter 2}

\section{LITERATURE REVIEW AND DESIGN FUNDAMENTALS}

\subsection{Brain Connectivity Analysis}

The human brain is a powerful and complicated machine whose functional activity can be viewed as a complex neuronal web of information flow and processing among its many regions. Years of investigation on how the brain works identify this advanced system as a modular neuronal machine that performs complex tasks through a myriad of interactions among its various units. Neuroscience investigations justify many important brain behaviors that verify these modular activities. A good example is the brain plasticity when an injury happens in which the brain adapts itself with the help of these networks. As another example, the brain cognitive responses can be well interpreted by functional brain networks.

The interplay between the different neuronal regions commonly referred to as brain connectivity maps constitute a new platform to determine and assess complex aspects of brain's behavior as revealed through these maps. Brain mapping studies define the brain as a modular machine with the segregated units that each is responsible for a specific part of the body and specific tasks. The association between these distinct areas is so complicated that it is one of the most challenging tasks in this research endeavor (Friston, 2011). The notion of brain connectivity comes to life when we want to look at the interactions between these segregated neuronal structures for doing a particular task or in observing the effects 
of a specific disease condition. In general, brain connectivity is realized in the following contexts (Sakkalis, 2011):

- Structural connectivity, which refers to patterns of anatomical links

- Functional connectivity, which indicates temporal statistical dependencies

- Effective connectivity, which investigates the causal interactions

Structural connectivity considers the physical connections between neurons, and it is referred to the fiber pathways linking the cortical regions of the brain known as white matter. Magnetic Resonance Imaging (MRI) and Diffusion Tensor Imaging (DTI) are the most useful modalities to discover and investigate the actual anatomical links. Functional connectivity defines as the temporal interdependencies that realized as the statically significant relations obtained from the activity of various brain regions. Functional connectivity usually can be calculated with the help of modalities like LFP (Local Field Potential), EEG, MEG, PET, and fMRI. Causal influences that can be estimated between neuronal assemblies are called effective connectivity. The effective connectivity can be assessed by applying the data-driven techniques or the model-based methods to the signal recording of the activity of these different brain units (Sakkalis, 2011).

\subsection{A brief review of functional connectivity measures}

Several methods have been invented to measure the interdependence of brain cortical assemblies using electrophysiological data. These methods helped to explore and quantify 
the neural coordination of the brain when studying the behavior of a healthy brain or when considering a condition under a specific neurological disorder. There are many discussions comparing the benefits of various brain connectivity metrics, but the superiority of one or a group of methods over the rest has not been proven yet. In fact, the neural dependency measures should be selected based on the application domain, the goal of the study and the data type.

The connectivity attributes can be measured and categorized from different points of view. One aspect is whether we want to obtain only the statistical relation between the brain modules that yield the functional connectivity or include causation as an important factor as long as it can be extracted from the effective connectivity. Functional connectivity methods like correlation or coherence simply quantify the strength of neural relation without inferring any causation. In contrast, techniques like Granger causality or transfer entropy determine the influence of one neuronal assembly over other areas (Bastos and Schoffelen, 2016).

Furthermore, an important subdivision of connectivity methods is to address whether we are to analyze the signals in the time domain or conversion of the signal to the frequency domain is needed. According to the application domain, it is sometimes useful to investigate the electrophysiological interactions in a specific frequency band. In such cases, the signal is converted using the common frequency transformation techniques like Fourier decomposition, Wavelet and Hilbert transform alongside with bandpass filtering in order to separate the electrical activity in the desired frequency interval. 
Some connectivity techniques are calculated in a predefined framework with specific assumptions such as linearity. These types of methods are known as model-based methods. The correlation coefficient is a well-established model-based connectivity metric that evaluates the linear relation of the recorded electrical neuronal activity without considering their temporal structure. Different than the model-based methods, some model-free methods have introduced new ways for estimating the interdependence without any assumption. As an example, the mutual information method which applies information theory techniques to estimate the linear and nonlinear dependencies among the neural oscillations is a good example of the model-free technique. Granger causal modeling and transfer entropy are other good examples of effective connectivity which are model-based and model-free respectively.

The interaction between the neural assemblies is recorded as electrical oscillations in the form of electrophysiological data like EEG. Therefore, the neuronal relations reflected in the signal properties can be made available by applying modern computational techniques. In the case of EEG, the most informative properties are amplitude, frequency, and phase. However, there is evidence that the methods based on the signal amplitude could effectively represent the modular relations of the brain neurons, and show that phase synchronization based metrics are more suitable for analyzing the functional connectivity networks since such metrics highlight the synchrony of cortical modules rather than the signal amplitude relations (Sun et al., 2012; Sobayo et al., 2013). In addition, such techniques are more suitable to be tested statistically. 


\subsection{Phase coupling between two oscillations}

When two neural modules communicate, the simultaneous firing of action potentials in distinct areas can be measured as the phase coupling of the two electrophysiological signals. In fact, the signal phase is the indicator of the oscillator situation in relation to its baseline for that moment in time. Such a measure is known as an instantaneous phase. A periodic signal has the deterministic phase, while when the signal is changing over time, the phase would be a variable value. When we deal with time-varying signals, the phase coupling is defined differently. The phase synchrony among two time-varying signals is clearly defined by (Bastos and Schoffelen, 2016) as depicted in Figure 1. Based on this definition, the phase value would be the summation of instantaneous phase vectors over the signal time slot in a specific frequency, and the two signals will remain synchronized in phase as long as their phase difference remains within some specified limit. 
(A)

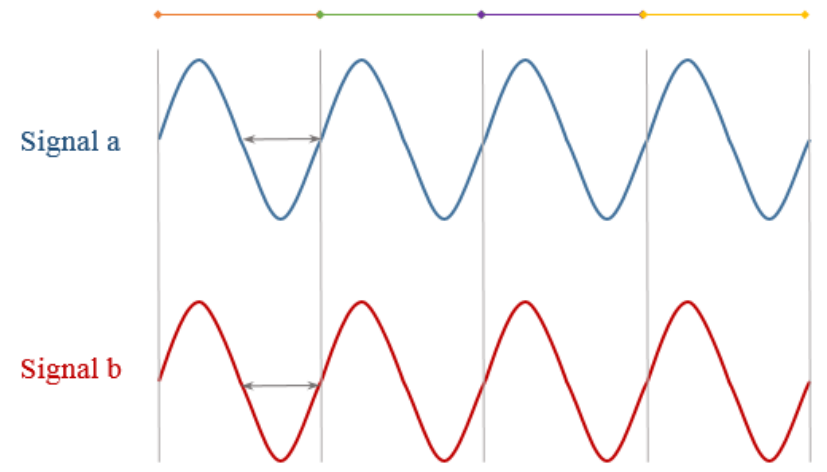

Zero phase difference between signal $a$ and signal $b$

(B)

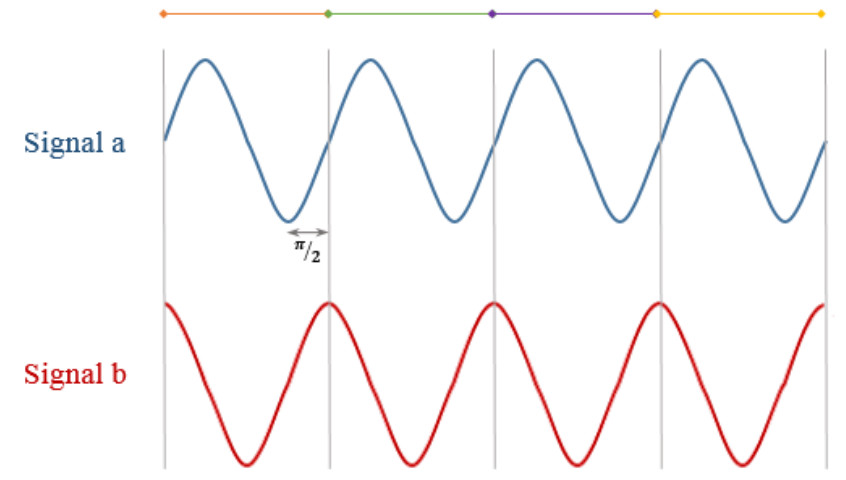

Signal a and signal $\mathrm{b}$ have $\pi / 2$ phase difference

(C)

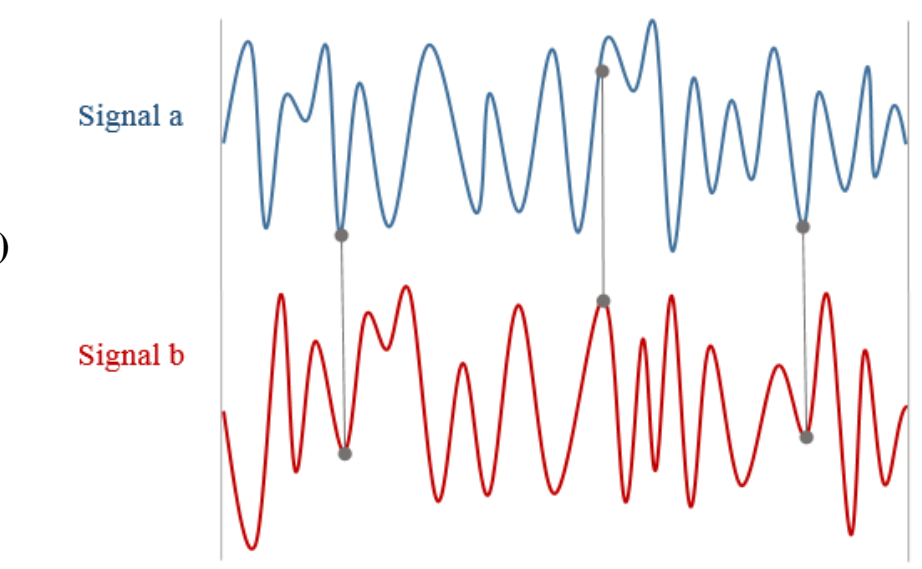

Signal a and signal $b$ have variable phase and consequently variable phase difference

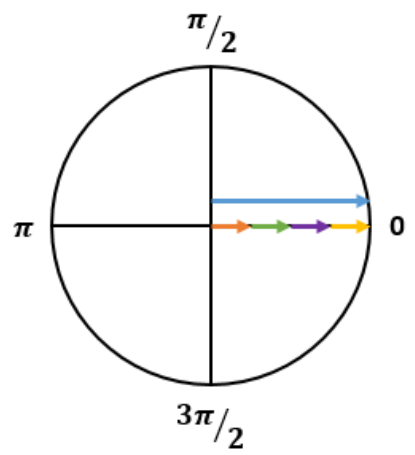

Total phase difference is one

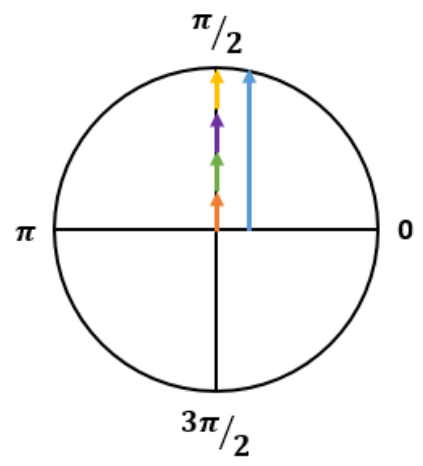

Total phase difference is one

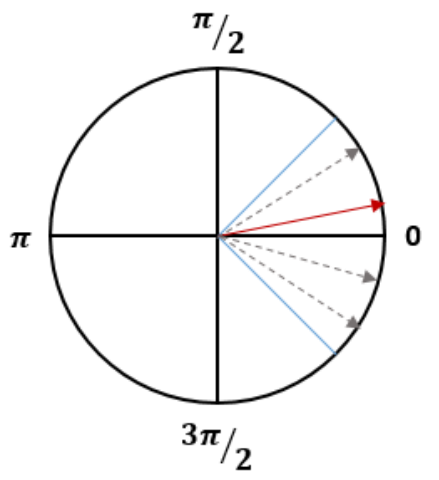

Figure 1. The definition of phase synchronization for two signals (A) Complete synchronization with zero phase difference in a specific frequency (B) Phase synchrony with phase difference of $\pi / 2$ in a specific frequency $(\mathrm{C})$ State of phase synchrony among two signal with variable phase. 


\subsection{Nonlinear methods for phase synchrony analysis}

Phase synchronization can be realized by a variety of methods, which most of them need the calculation of the phase synchrony in the frequency domain. Coherence, phase slope index, phase lag value, the imaginary part of coherency are popular methods that estimate the phase synchrony from the signal conversion to the frequency domain. In addition, linear methods can be applied to the signal or nonlinear techniques can be adopted instead albeit with more computational requirements (Stam et al., 2007; Kalitzin et al., 2007; Yifan Zhao et al., 2013). Some new methods have been developed that suggest the calculation of phase synchrony between two signals using the FFT signal conversion (Majumdar, 2006; Golshan et al., 2016). Since the complex and nonlinear nature of brain signals can be well explained as chaotic oscillators (Marwan et al., 2007; Adeli et al., 2007; Kurths et al., 2006), and due to the non-stationary features of the EEG signals, phase synchronization can be well estimated by applying recurrence-based methods to such chaotic systems (Lehnertz et al., 2009; Lakshmanan and Senthilkumar, 2011; Rangaprakash et al., 2013).

EEG signals, as the measurable behavior of the brain, inherit many complex attributes (Rangaprakash, 2014). Such attributes may be more easily discovered by using nonlinear operators (Acharya et al., 2011). Furthermore, the dynamic behavior of brain signals directs the analysis more towards modeling the brain as a chaotic system (Adeli et al., 2007; Rangaprakash, 2014). The chaotic model of the brain can be a helpful platform to extract the higher-order information by means of nonlinear operators. As per the method presented 
in (Kurths et al., 2006), phase synchronization of two signals can be obtained by modeling them as chaotic oscillators.

The synchronization phenomena among nature-derived signals inherit some behavioral features. EEG signals, as the measurable behavior of the brain, hides many complex attributes inside (Rangaprakash, 2014). Such attributes, which may be more easily discovered by using nonlinear operators, provide important information that helps these natural mechanisms to be more recognized (Acharya et al., 2011). Various states of synchronization are defined for nonlinear oscillators. Any two nonlinear oscillators, with similar state variables, are considered completely synchronized. While variables of one system follow the state variables of the other with a shift in time, a lag in synchronization is recognized. General synchronization is also defined when there is a known relationship between the state variables of two systems (Lehnertz et al., 2009). As a matter of fact, the concept of general synchronization investigates the conformity of one chaotic attractor on the other (Sakkalis, 2011). This process evaluates the synchronization strongly even when the signals lack stationarity. The system attractor can be built by the measurable property of the system which in case of the brain it will be through the EEG signal. The brain attractor can be reproduced by the time embedding theory. The resulted attractors are then investigated for the neighborhood mapping (recurrence) that yields to the recurrence plot. The recurrence plots would be quantified to obtain the signal couplings and in some cases the direction of the synchrony (Lehnertz et al., 2009). 
When applying nonlinear methods, data compatibility needs to be examined since a linear correlation or filtered noise in the data might lead to unexpected outcomes (Natarajan et al., 2004; Schreiber and Schmitz, 2000; Puthanmadam Subramaniyam, 2016). Surrogate data testing is a reliable statistical method that helps evaluate the existence of nonlinearity as well as confirming the significance of the obtained results (Marwan et al., 2007; Natarajan et al., 2004; Gautama et al., 2003). This method works based on generating surrogates with similarities to the original data but also with some randomness in its nature (Junfeng Sun et al., 2012). The method tests the desired metric against the null hypothesis of no significant difference among results between original and surrogate data. In fact, the more similar the surrogate is to the original data, the more reliable the results will be (Natarajan et al., 2004). Various techniques are available to generate a surrogate. Surrogate data can be generated easily by shuffling the components of time series (e.g., random shuffling) or applying more advanced methods based on the Fourier transform to maintain some spectral similarities with the original signal (Puthanmadam Subramaniyam, 2016). Among all methods, to create a surrogate data, the iterative Amplitude Adjusted Fourier Transform (iAAFT) remains the most reliable and most popular method (Puthanmadam Subramaniyam, 2016). The surrogate data testing method has been used in most of the recurrence-based studies, but in this study, we have used this useful statistical test in a novel manner to find suitable parameters that lead to improved results. 


\subsection{Epilepsy as a network disease}

Epilepsy is a chronic neurological disorder distinguished by the abnormal synchronous activity of brain neurons. This abnormal activity, which is concurrent neuronal electric discharges, causes a nervous attack known as a seizure (Fisher et al., 2005). Epileptic seizures, which are clinically recognized as ictal events, are categorized into two major groups of focal and generalized based on the type of seizure origin. In general, the genesis of a focal seizure is limited to a single hemisphere while a generalized seizure has distributed sources engaging both hemispheres. A focal seizure can be seen in 60 percent of people with epilepsy. In most of the focal epilepsy cases, seizure arises from the temporal lobe creating a wide class of syndromes known as Temporal Lobe Epilepsy (TLE) (National Institute of Neurological Disorders and Stroke (US). Office of Communications and Public Liaison, 2015).

Epilepsy is thus mainly characterized by its seizures or ictal events during EEG recordings. However, there are between seizures some events known as interictal epileptiform discharges (IEDs), which carry significant attributes of this brain disorder. Due to the unpredictability of seizures, a thorough investigation of interictal events could enhance our understandings and management of this disease (Smith, 2005).

Due to its simplicity and noninvasive nature, scalp electroencephalography (EEG) recordings remain to this day the first recording modality utilized for diagnosis of epilepsy (Smith, 2005; Acharya et al., 2013). Practical considerations, cost-effectiveness, and high temporal resolution make of the EEG recording modality a convenient platform to 
investigate the epileptogenesis of the disease by studying ictal and interictal episodes in patients with epilepsy (Smith, 2005). In many studies, interictal EEG intervals were frequently utilized to locate the epileptogenic zone (EZ) in focal epilepsy (Mégevand and Seeck, 2018). In (Mégevand et al., 2014), IEDs were used to locate the epileptic source of the high-density scalp EEGs in a population of 38 focal epileptic individuals, and results were precisely correlated with the epileptogenic foci found through seizure onset. However, authors in (Bartolomei et al., 2016) differentiated the brain areas affected by IEDs and regions that initiated the seizure and concluded the agreement between these zones in $75 \%$ of their study population.

Recent studies consider epilepsy as a network disease, which means that in spite of the epileptic focus, there is a network of neuronal activities that contribute to the unusual behavior of the disease (Lehnertz et al., 2009; Wilke et al., 2011; Peng Xu et al., 2014). It is observed that IEDs as interictal abnormalities make alternations to the default network of the brain (van Diessen et al., 2016). As an example, effects of spikes in connectivity of mesial temporal lobe epilepsy were tested using frequency domain analysis of EEG signals in (Bettus et al., 2008), which resulted in increased connections in the presence of spikes. A model-based probabilistic connectivity analysis was thus performed during spike-wave discharges in (Taylor et al., 2013), and authors concluded that higher synchrony, larger amplitude, and complex spatial profile were related to the spike activity. In (Coito et al., 2015), the variation of epileptic networks during interictal spikes were compared in the right and left temporal lobe epilepsy by applying the electrical source imaging analysis to the high-density EEG recordings. Authors of this study have determined that there was a 
significant difference in connectivity networks of these two disease types. As another application, directed functional connectivity networks, which were obtained from highdensity EEG, were used to differentiate between the three groups of right temporal lobe epilepsy (RTLE), left temporal lobe epilepsy (LTLE) and normal controls (Verhoeven et al., 2016). In (Staljanssens et al., 2017), functional connectivity helped in electrical source imaging to precisely locate the seizure onset area during ictal events. In (Amini et al., 2011), a directed graph analysis method was adopted to locate the origin of the IED activity using intracranial data. Results were corroborated with the clinically discovered epileptogenic source. In (Meesters et al., 2018), a network analysis of IEDs over stereotactic EEG data proved a correlation among spike cluster's location and seizure onset zone.

Source localization using scalp EEG is a technique commonly used to find the epileptogenic area in patients for presurgical evaluation and planning. At this stage, the epileptic focus needs to be located as accurately as possible. To achieve this goal, highdensity EEG and source level directed functional connectivity proved to be most useful (Sohrabpour et al., 2016; Coito et al., 2016). However, researchers need to deal with head model selection and inverse solutions that inherently face practical limitations and introduce errors to the 3-D localization process (Hosseini et al., 2018; Wilke et al., 2008; Cheung et al., 2012).

Graph theory analysis is an emerging field that is used for the interpretation of the functional brain networks. In fact, graph theory with the help of dynamical system analysis 
and statistics creates the new field of the modern network science, which appear to elucidate the ambiguity of the brain network perception (Stam, 2014). Brain connectivity matrices can be represented as an adjacency matrix of a graph (Wilke et al., 2011; Stam and Reijneveld, 2007). Different network topologies are defined depicting normal or a specific condition of a brain disorder (Stam, 2014). A healthy brain has adapted an optimized combination of local information processing with efficient global data transfer between modules. This attribute that is known as small-world network topology will be damaged when the brain is engaged with an abnormality like epilepsy (Ponten et al., 2009). The two main graph theory attributes of the network that defines the network topology are the clustering coefficient which indicates the local information processing and the shortest path length that represents the global information transfer (Stam, 2014). 


\section{Chapter 3}

\section{NONLINEAR ESTIMATION OF THE BRAIN FUNCTIONAL CONNECTIVITY}

\subsection{Introduction}

In the category of nonlinear methods for EEG analysis, recurrence plots (RP) and recurrence quantification analysis (RQA) are common methods (Sakkalis, 2011; Marwan et al., 2007). As a further extension, RPs were utilized to estimate the phase synchronization (PS) between the dynamics of two complex systems (Rangaprakash, 2014). We used the method that is called correlation between probabilities of recurrences (CPR) to calculate the PS among signals of the EEG electrodes (Lakshmanan and Senthilkumar, 2011). In fact, the CPR method finds the synchronized periodic rhythms hidden in the signal and estimates the amount of phase synchrony between them (Marwan et al., 2007). This characteristic makes this method a strong candidate for analyzing natural chaotic oscillators like brain signals. It is proven that brain signals, like many other natural occurrences, are neither periodic nor random but rather chaotic.

Recurrence is a basic property that is quite useful in assessing nonlinear and chaotic systems, especially when looking at the phase space. It is expressed as the return (revisit) of system trajectories to a specific neighborhood or site after a period of time. Recurrence is thus the basis for analyzing phase synchrony between two nonlinear systems. The brain can be considered as a nonlinear chaotic system. Therefore, it is suitable to investigate the behavior of brain dynamics (phase) in a reconstructed multi-dimensional space. 


\subsection{Functional Connectivity based on the Probability of Recurrence}

The signal trajectory in the phase space can be easily rebuilt using time delay theorem as expressed in (Lakshmanan and Senthilkumar, 2011). As per this theory, the signal $X=$ $\left[x_{1}, x_{2}, x_{3}, \ldots, x_{N}\right]$ with $N$ entries, reconstructed into a trajectory $y_{i}$, in phase space, can be defined as follows:

$$
\begin{gathered}
y_{i}^{T}=\left[\begin{array}{llll}
x_{k} & x_{k+t} x_{k+2 t} & \cdots & x_{k+(m-1) t}
\end{array}\right] \\
m \geq 1, t \geq 1, i=1, \cdots N \text { and } k=1, \cdots, N-(m-1) t
\end{gathered}
$$

Where $m$ defines the embedding dimension, $t$ represents the time delay, and $y_{i}$ defines the points on the trajectory. The trajectory preserves the original properties of the EEG signal; therefore, the spike activities were reflected in these constructed routes (Guevara et al., 2005).

The brain signal trajectories, like any other chaotic dynamics, have recursive behavior. The trajectory returns to its neighborhood after a passed time, thus establishing a recurrence. The amount and pattern of recurrences explain the signal behavior (Guevara et al., 2005). Since a direct investigation of trajectory behavior in phase space is not feasible, recurrence maps are developed to assess such behavior (Mercedes et al., 2005). Calculation of recurrence is consequently formulated as in (2)

$$
R_{i, j}=\Theta\left(\varepsilon-\left\|y_{i}-y_{j}\right\|\right)
$$


Based on this relation, the recurrence between points $i$ and $j, R_{i, j}$, is said to occur when the Euclidean distance between these two points in the trajectory is less than a set threshold $\varepsilon$. The function $\Theta$ is a Heaviside step function making the resulted value, 0 (for no recurrence), or 1 (when recurrence occurs). Calculation of recurrence for all points of the trajectory leads to the matrix of the recurrence plot.

Various behaviors in trajectories create a variety of specific patterns in the RPs (Marwan et al., 2007). These patterns are extracted by applying the Recurrence Quantification Analysis method and are used to characterize the different signal states. The periodic rhythms are represented as a long diagonal line in the RPs, where the constant vertical distances indicate the period of the signal (Marwan et al., 2007). In the case of chaotic systems, the diagonal lines are interrupted, and the vertical lines are not consistent thus representing different periodicities that are concealed in the original signal. If two dynamic systems develop a PS, the vertical distances in both RPs are said to be simultaneous (Marwan et al., 2007). The RPs diagonal length and the vertical line can be utilized to calculate the probability of recurrence to estimate the PS among electrodes of one EEG segment. This probability of recurrence can be designated as a generalized autocorrelation of the original signal by calculating the number of returns of the trajectory to a specific neighborhood as defined in (3) (Marwan et al., 2007):

$$
p(\tau)=\frac{1}{N-\tau} \sum_{i-1}^{N-\tau} R_{i, i+\tau}
$$


Where $\tau$ is the time delay and $N$ is the original signal length. The $p(\tau)$ represents the system recurrence (diagonal lines in RPs) based on time delays. If two systems develop phase synchronization, the peaks of $p(\tau)$ of the two signals will coincide. Using this notion, the value of phase synchronization between two-time series can be calculated using the following equation:

$$
C P R_{i, j}=\frac{\sum_{t=\tau_{e}}^{\tau_{m}-1}\left\{\left(p_{i}(\tau)-m_{i}\right) \cdot\left(p_{j}(\tau)-m_{j}\right)\right\}}{\sigma_{i} \sigma_{j}}
$$

$C P R_{i, j}$ defines the correlation between the probabilities of recurrences, with $m_{i}$ being the mean and $\sigma_{i}$ being the standard deviation of the $p_{i}(\tau)$. The CPR values range from zero when there is no PS between two signals and reaches a value of 1 for complete PS between the two signals (Rangaprakash and Pradhan, 2014). Calculating all mutual CPR values among all electrodes of one EEG segment establishes the phase synchronization matrix of that EEG segment as in (5).

$$
C P R_{N \times N}=\left[\begin{array}{ccc}
C P R_{1,1} & \cdots & C P R_{1, N} \\
\vdots & \ddots & \vdots \\
C P R_{N, 1} & \cdots & C P R_{N, N}
\end{array}\right]
$$

Considering the values of phase synchronization as the strength of connections between brain modules in EEG signals, the brain connectivity matrix is obtained. In fact, two electrodes are considered connected, if the CPR value is high (closer to 1) and are deemed not connected if the value of phase synchronization is low (around 0) (Rangaprakash, 2014). Based on the literature, the CPR value of 0.5 or 0.6 indicates that both time series 
had started to develop phase synchronization (Lakshmanan and Senthilkumar, 2011; Schelter et al., 2006).

\subsection{Parameter Selection and Significance Evaluation}

To achieve a reliable connectivity matrix, optimal selection of suitable parameters (time lag, embedding dimension and recurrence threshold) is essential. Various methods have been suggested in the literature to find appropriate parameters. A very small value for the time lag causes to capture no new information in the successive sampling and creates a redundant situation, while a too large value results in information loss (Puthanmadam Subramaniyam, 2016). Mutual information is one of the most popular methods used to determine the optimal time lag (Puthanmadam Subramaniyam, 2016; Zbilut and Webber, 2006). Mutual information is the value that is sensitive to the change of the state of the brain. The suitable time lag can be found at the occurrence of the first local minima of the auto-mutual information curve of the signal (Puthanmadam Subramaniyam, 2016; Zbilut and Webber, 2006; West, 1991). On the other hand, the embedding dimension should be large enough to capture the dynamics of the signal but with the caution that very large embedding dimension would make the calculation expensive (Rangaprakash, 2014; Zbilut and Webber, 2006). The $\mathrm{K}^{\text {th }}$ nearest neighbor method has been used extensively to find an optimal embedding dimension, (Puthanmadam Subramaniyam, 2016; West, 1991). The recurrence threshold is the parameter that affects the occurrence of the recurrence. We have 
considered the value of $10 \%$ of space average diameter for such recurrence threshold (Marwan et al., 2007).

Despite the fact that the aforementioned methods are used to find optimal parameters, using these factors does not guarantee to obtain significant connectivity matrices. To assure the significance of the calculated results, we used the surrogate data testing technique to suggest an algorithm for choosing the proper parameters and evaluate the significance of the connectivity matrices. The method works based on generating surrogates of original signals and test for the null hypothesis of similarity between two connectivity matrices generated from original and surrogate signals. Rejection of the null hypothesis is an indication that the connectivity matrix is significant. To generate the surrogate data, we have used the iAAFT method mentioned earlier, which showed more credible results (Gautama et al., 2003). The number of surrogates calculated for each EEG segment depended on the confidence level we needed for the test. According to the literature (Gautama et al., 2003), for the $\alpha$ level of significance the minimum number of surrogates $M$, as defined in (6), can be estimated as follows:

$$
M=\frac{2 K}{\alpha}-1
$$

Where $\mathrm{K}$ is an integer normally chosen as 1 for the sake of simplicity. Hence, for a twosided test with a $95 \%$ confidence interval, $\alpha$ will be 0.05 , and $\mathrm{M}$ will yield 39 surrogates that would be needed in that case. 


\subsection{Brain Network Interpretation Using Graph Theory}

Graph theory is a powerful tool in the interpretation of functional brain networks. In brain network analysis the connectivity matrix resembles a graph with electrodes as nodes and connectivity values as links. This graph can be weighted, where connectivity strength defines the link weights, or it can be unweighted where a link represents the existence of a connection. A graph can also represent whether there is a directional or unidirectional relation. A unidirectional graph has a symmetrical adjacency matrix, while directed links are characterized by an asymmetric matrix. Many graph theory measures have been created for the sake of better exploration of the brain networks. Here we overview measures that have been used in the network analysis of this thesis.

- Node degree $(D)$ : A degree of a node defines the sum of the connected links to the node.

- Average node degree $(A D)$ : The degree average of all nodes in a graph.

- Link density $(L D)$ : The link densities in a graph that outlines the sum of available edges over the number of possible links in the graph.

- Rich club metric $(R C M)$ : the notion of rich club metric is based on the centrality concept. Centrality is the measure that shows the importance of a node in relation to the other nodes. It is realized as degree centrality, closeness centrality or betweenness centrality. A hub is a node with a high value of centrality. A collection of highly connected hubs is considered a rich club. 
- $\quad$ S metric $(S M)$ : it is defined as the sum of the product degrees across all edges.

- Algebraic connectivity of a graph $(A C)$ : This feature refers to the spectral analysis of the graph, which is based on the Laplacian of the adjacency matrix. It is defined as the second smallest eigenvalue of the Laplacian.

- Graph radius $(G R)$ : The maximum distance to any other vertex is defined as the eccentricity for each node. The graph radius is considered as the minimum node eccentricity.

- Clustering coefficient $(C)$ : If two nodes are connected, then they are called neighbor nodes. The clustering coefficient of node $i$ is the probability that its neighbor nodes are also connected. The coefficient clustering is a measure of local connectivity (Stam, 2014; Ponten et al., 2009)

- Shortest path length $(L)$ : it is the smallest number of links that connects two nodes. The average shortest path length is the average $L$ over all pair of nodes. (Ponton, Indication of)

- Small world index: a network with the small world characteristics has a high clustering coefficient $(C)$ with the small value of the shortest path length $(L)$. 


\section{Chapter 4}

\section{CLUSTERING BY FUNCTIONAL CONNECTIVITY}

\subsection{Introduction}

In this chapter, a novel nonlinear data-driven algorithm is proposed to delineate Electroencephalogram recordings as either coming from controls or patients with epilepsy. This method uses the probability of recurrence and the correlation between electrodes to extract the phase synchronization and the functional connectivity maps of the brain from interictal EEG data recordings. This newly proposed algorithm utilizes probabilistic clustering by extracting graph theoretical features from the calculated functional

connectivity matrices. Results reveal that brain connectivity networks of epileptic and control populations show statistically significant differences $(\mathrm{t}(340)=-37.4771, \mathrm{p}<0.01)$ between them. Performance results show an accuracy of $92.8 \%$ with a sensitivity of $85.7 \%$ and a specificity of $100 \%$ when tested on 14 subjects. These preliminary results confirm that this method can be used to enhance and validate the diagnosis of epileptic patients from controls using non-invasive scalp EEG signals.

\subsection{Material and Methodology}

Multichannel scalp EEG signals from seven control subjects and seven patients diagnosed with epilepsy were recorded using referential montage following the 10-20 electrode placement protocol. The study was approved by the Institutional Review Board (Protocol 
number: IRB-052708-03) and consent forms were provided to the subjects or their legal representatives. Nineteen electrodes (C3, C4, O1, O2, Cz, F3, F4, F7, F8, Fz, Fp1, Fp2, P3, P4, Pz, T3, T4, T5, T6) were used for the purpose of this study. Recordings are digitized with sampling rates of 200 and $512 \mathrm{~Hz}$, indistinctly across both groups. Scalp EEG recordings were broken into segments mostly 10 seconds long. Segments were visually inspected, and a total of 134 segments were chosen from the whole population. The EEG segments were free of artifacts and ictal events. Table 1 shows the subject's information, and Figure 2 describes the flowchart of the algorithm, which was implemented using the MATLAB environment.

\subsection{Preprocessing}

To improve signal to noise ratio (SNR) and reduce the effect of unwanted sources, EEG data were preprocessed by filtering each electrode recording with a digital infinite impulse response (IIR) notch filter $(60 \mathrm{~Hz})$ to remove power line interference, and a 4th order Butterworth zero-phase digital band-pass filter with a range of $[0.5,70 \mathrm{~Hz}]$. Baseline was also removed from the contributed data. 
Table 1. Test Population Information

\begin{tabular}{|c|c|c|c|c|}
\hline Subject ID ${ }^{*}$ & Age & Gender * & Diagnosis & Sampling rate $(\mathrm{HZ})$ \\
\hline$C 1$ & 12 & M & - & 200 \\
\hline$C 2$ & 15 & $\mathrm{~F}$ & - & 512 \\
\hline C3 & 12 & M & - & 200 \\
\hline C4 & 15 & $\mathrm{~F}$ & - & 512 \\
\hline$C 5$ & 10 & M & - & 512 \\
\hline C6 & 18 & $\mathrm{~F}$ & - & 512 \\
\hline$C 7$ & 8 & M & - & 200 \\
\hline$P 1$ & 10 & $\mathrm{~F}$ & L.T. lobe dysplasia & 200 \\
\hline$P 2$ & 7 & $\mathrm{~F}$ & L. Frontal region & 512 \\
\hline$P 3$ & 7 & M & LF Posterior frontal lobe & 512 \\
\hline$P 4$ & 14 & M & Generalized & 512 \\
\hline P5 & 4 & M & R Frontal to Centro temporal & 512 \\
\hline P6 & 2 & $\mathrm{~F}$ & LT. (posterior) & 512 \\
\hline$P 7$ & 8 & M & R. Parietal & 200 \\
\hline
\end{tabular}

* Controls and Patients are labeled as " $\mathrm{C}$ " and "P" respectively

* M: Male, F: Female 


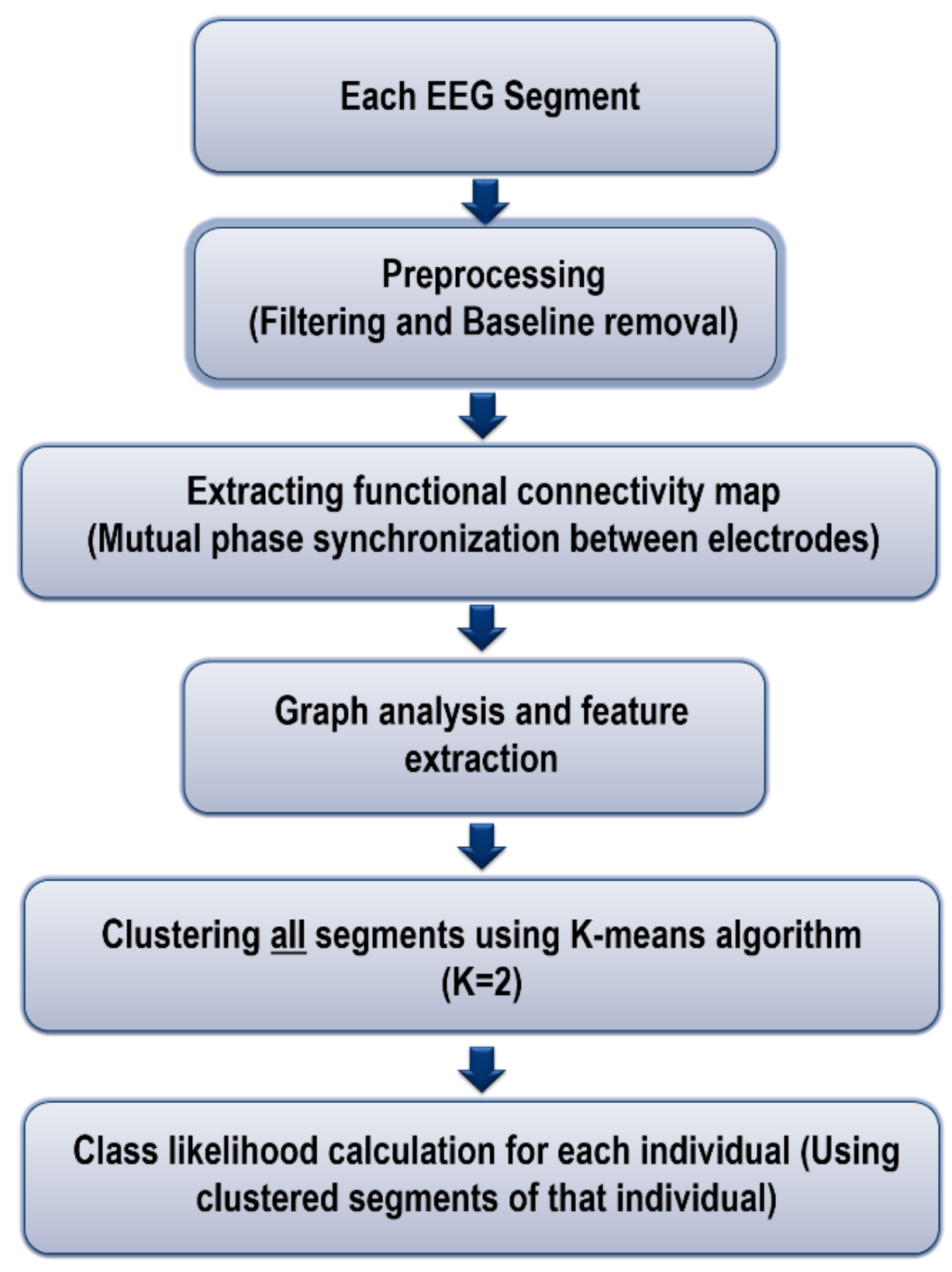

Figure 2: Flowchart for the suggested algorithm 


\subsection{Functional Connectivity and Feature Extraction}

The connectivity matrix is considered as the weighted undirected adjacency matrix of a graph. The EEG electrodes represent the nodes of the graph, while the edges show the connection strength between the nodes (Sargolzaei et al., 2013). The hypothesis is to test for the existence of statistically significant differences among the functional connectivity matrices of healthy brains and those of diseased ones and ultimately determine whether the hypothesized difference could be used to delineate epileptic patients from healthy control subjects. For this purpose, a set of 6 graph theory features, as shown in Table 2, were extracted from the graph obtained for each segment.

\subsection{K-means Clustering}

Total extracted features from 134 segments are fed to a k-means clustering algorithm. The clustering process was totally unsupervised without any training, and each segment was categorized in one of the two groups that later were labeled as epileptic or control $(\mathrm{K}=2)$. Considering the results for contributed segments of each EEG recordings, the probability of belonging to each group was calculated for each individual using (6).

$$
P_{i x}=\frac{N_{i x}}{T_{i x}}
$$

Where $\mathrm{P}_{\mathrm{ic}}$ is the calculated probability for each individual that show the degree of belonging

of individual $i$, and $x$ could be $E$ for epileptic or $C$ for controls. The term $N_{i x}$ is the number 
of segments clustered in a group $x$ and $T_{i x}$ is the total number of segments. Individuals will be considered to a group in which the corresponding probability is more than $50 \%$.

Table 2: Brief Description of Extracted Features

\begin{tabular}{ll}
\hline FEATURE & DESCRIPTION \\
\hline$L D$ & Link Density of the Graph \\
$A D$ & Average Degree of a node \\
$R C M$ & Rich Club Metric \\
$S M$ & S Metric \\
$A C$ & Algebraic Connectivity of a graph \\
$G R$ & Graph Radius \\
\hline
\end{tabular}

\subsection{Results and Discussion}

To validate the hypothesis of the proposed method, which is to delineate between functional connectivity graphs of epileptic and controls, a two-sided two-sample student ttest was implemented on the average functional connectivity matrices of the two groups. A Null hypothesis of the mean difference of functional connectivity of healthy and epileptic individuals resulted in a significant difference between the two groups $((\mathrm{t}(340)=-37.4771$, $\mathrm{p}<0.01)$ ). Figure 3 shows the head map plot of average connectivity matrices for epileptic and control subjects. In order to properly visualize the connections, only strong connections (higher than a specified threshold in strength) are kept and shown in the head map graph.

In Figure 3, the head map plot of epileptic patients represents significantly higher and stronger connections compared to the control subjects under both thresholds considered. This observation strongly supports the proposed hypothesis that the epileptic brain has 
more connections in interictal stages compared to the control brain. In Figure 4, the number of connections is compared between two groups based on different thresholds. As it can be observed, the number of connections for epileptic patients validates the hypothesis that abnormal networks will show more activation in different areas of the brain. Consideration of more EEG segments will increase the accuracy of the final clustering.

(a)
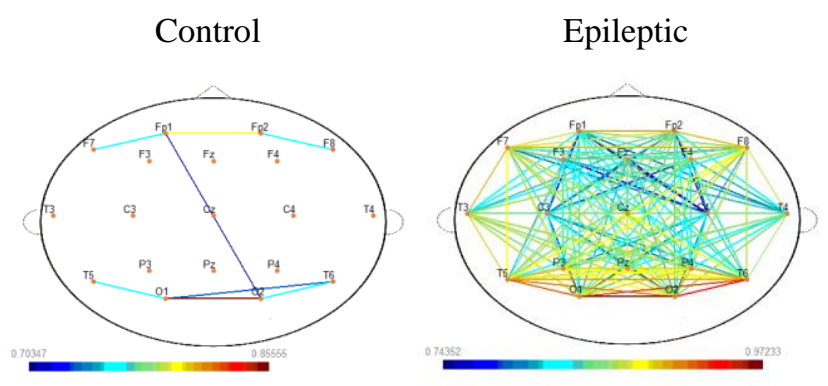

(b)

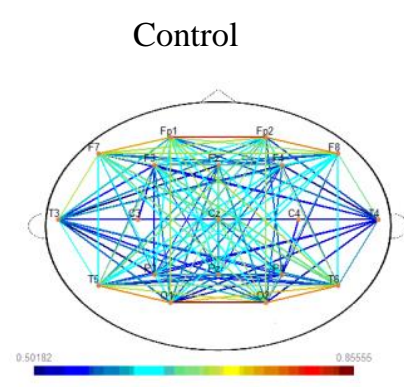

Epileptic

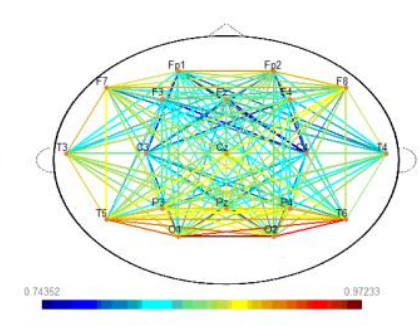

Figure 3: Connectivity head map of average connectivity matrices for controls and epileptic patients (a) higher $30 \%$ connections and (b) higher $50 \%$ connections

Table 3 shows the final result of clustering and the class likelihood for each subject. To assure the consistency of results, the experiment is repeated 1000 times. The suggested 
algorithm shows satisfactory results with high specificity of $100 \%$, accuracy of $92.8 \%$ and sensitivity of $85.7 \%$. Comparing this procedure with the method presented in (Sargolzaei et al., 2013), higher accuracy has been achieved while fewer features have been considered.

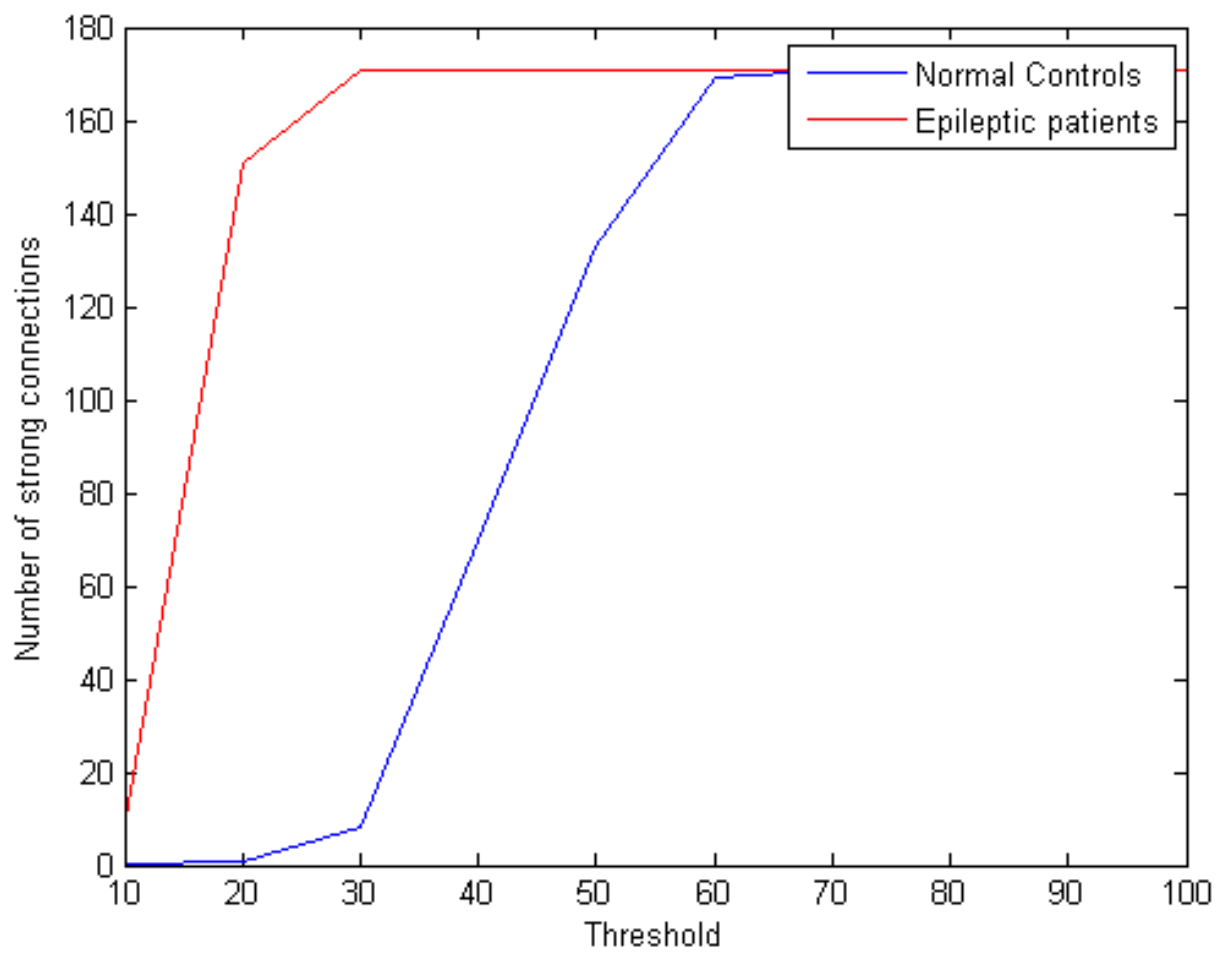

Figure 4: Number of strong connections based on different thresholds for average connectivity matrices 
Table 3: Clustering results

\begin{tabular}{|c|c|c|c|c|}
\hline Subjects ID & $\begin{array}{c}\text { No. of } \\
\text { Segments }\end{array}$ & $P_{i E}^{*}$ & $P_{i C}^{*}$ & Subject labeled as \\
\hline$C 1$ & 10 & $10 \%$ & $90 \%$ & Healthy \\
\hline$C 2$ & 10 & $0 \%$ & $100 \%$ & Healthy \\
\hline$C 3$ & 10 & $0 \%$ & $100 \%$ & Healthy \\
\hline C4 & 10 & $10 \%$ & $90 \%$ & Healthy \\
\hline C5 & 4 & $0 \%$ & $100 \%$ & Healthy \\
\hline C6 & 10 & $0 \%$ & $100 \%$ & Healthy \\
\hline$C 7$ & 10 & $20 \%$ & $80 \%$ & Healthy \\
\hline$P 1$ & 10 & $100 \%$ & $0 \%$ & Epileptic \\
\hline$P 2$ & 10 & $100 \%$ & $0 \%$ & Epileptic \\
\hline$P 3$ & 10 & $100 \%$ & $0 \%$ & Epileptic \\
\hline$P 4$ & 10 & $90 \%$ & $10 \%$ & Epileptic \\
\hline P5 & 10 & $100 \%$ & $0 \%$ & Epileptic \\
\hline P6 & 10 & $100 \%$ & $0 \%$ & Epileptic \\
\hline P7 & 10 & $30 \%$ & $70 \%$ & Healthy \\
\hline Accuracy & $92.8 \%$ & & & \\
\hline Sensitivity & $85.7 \%$ & & & \\
\hline Specificity & $100 \%$ & & & \\
\hline
\end{tabular}

* Probability of being epileptic

* Probability of being healthy 


\subsection{Conclusion}

Using scalp EEG, the delineation between patients with epilepsy and healthy controls is hypothesized to be related to their brain connectivity patterns. These patterns can be extracted using phase synchronization between electrodes. The algorithm implemented analyses some inherent characteristics of the EEG recordings by mapping the signal in phase space. The phase synchronization between electrodes, for a given EEG segment, addresses the connectivity maps of the brain, as connected electrodes are found to be more synchronized in the phase domain. The results strongly support the proposed hypothesis, since connectivity maps of epileptic patients were showing stronger connections in comparison to the healthy control group. The algorithm made use of the unsupervised kmeans clustering method for classification. This method simplifies the classification process since it does not need any training. The results obtained show significant differences among connectivity matrices between the two groups. As future work, this method will be tested on a larger population, so a higher accuracy can be obtained. This fully automated method can assist physicians as a validation tool to augment the prospects for the diagnosis of epileptic patients. 


\section{Chapter 5}

\section{CONNECTIVITY MAPS OF DIFFERENT TYPES OF EPILEPTOGENIC PATTERNS}

\subsection{Introduction}

EEG functional connectivity maps, showing the interactions between brain areas in context to the placement of electrodes, were used for the investigation and comparison of three different types of epileptiform activity defined as a single spike, spike followed by a slow wave and repetitive spike. A nonlinear data-driven method was used to extract connectivity matrices that helped to identify network synchronization based on the number of connections for all brain regions, as represented by the 10-20 EEG system. This quantification was used to assess these three types of spike patterns in relation to the type of seizure, focal or generalized. Results showed some differences between connectivity patterns of single spikes related to focal epilepsy and connectivity patterns of repetitive spikes related to generalized epilepsy. The statistical variance analysis reported a significant difference (P-value $\ll 0.001)$ between single spike connectivity maps and other spike types. The results obtained, augment the prospects for diagnosis and enhance recognition of disease type via EEG-based connectivity maps. 


\subsection{Material and Methodology}

Scalp EEG signals are recorded using the 10-20 international standard montage with a sampling frequency of 200Hz. Nineteen electrodes (including Fp1, F7, T3, T5, O1, F3, C3, P3, Fz, Cz, Pz, Fp2, F8, T4, T6, O2, F4, C4, P4) were used to collect EEG data from a male patient diagnosed with generalized epilepsy and a female patient diagnosed with focal epilepsy having partial complex seizures. These initial findings will be included in our assessments of 30 patients in an upcoming EEG-triggered fMRI study, where the 3D source localization based on the 10-20 montage are validated through the BOLD effect seen in the spike-triggered fMRI. The recorded EEG included both ictal and interictal events, but we use only interictal segments for this study. The research procedure was approved by the Institutional Review Board of Florida International University (Protocol number: IRB-150247) and the consent was received from all subjects.

Thirty segments of one-second duration containing three different spike patterns were selected as epileptic biomarkers. An equal number of segments were considered for each spike group that includes a single spike, spike and slow wave (complex spike) and repetitive spikes. Figure 5 shows the sample spike patterns of one electrode for each group. MATLAB software is used for processing the collected data. 


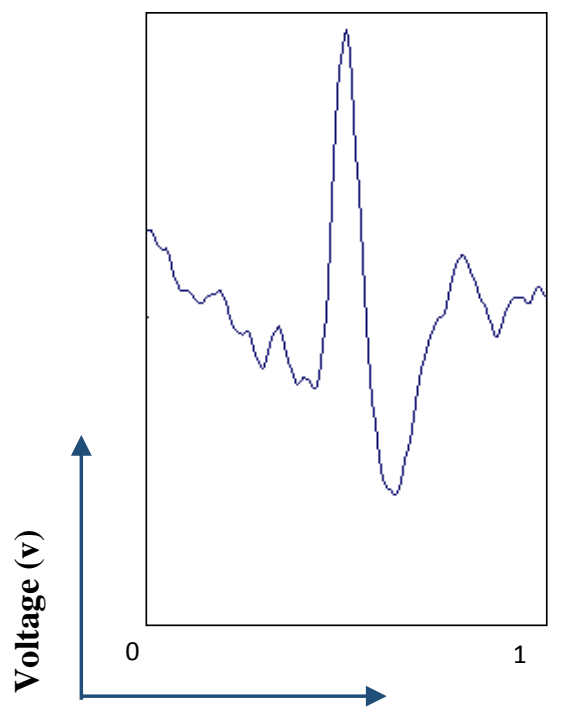

Time (s)

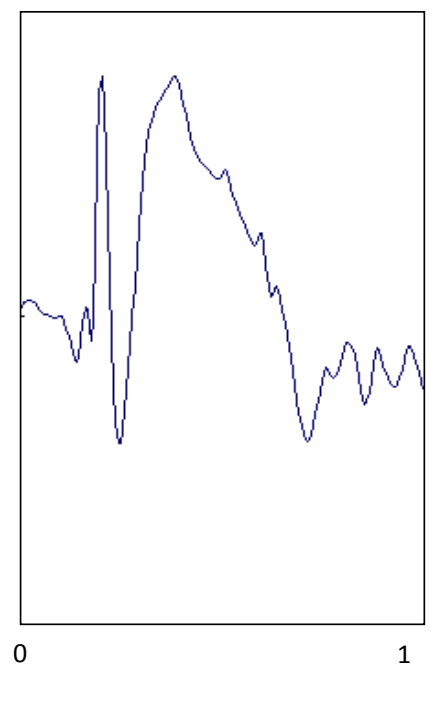

(b)

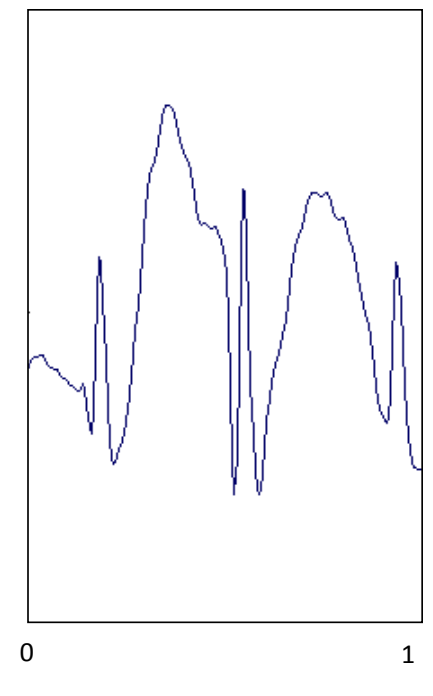

(c)

Figure 5: Sample of three epileptiform patterns in one electrode segment: (a) Single spike, (b) Complex spike (c) Repetitive spike

\subsection{Preprocessing}

To minimize the effect of unwanted noise, the EEG signals were preprocessed before segmentation. All EEG data were filtered by an IIR notch filter; with notch frequency of $60 \mathrm{~Hz}$, and a 4th order Butterworth zero phased digital filter with a passband of $[0.5,70$ ]Hz. The baseline of the signals was removed, and the recordings were changed to average montage (Rajaei et al., 2015). All segments of one group were aligned in order to place the spike peak in the same position for all segments. 


\subsection{Functional Connectivity Maps}

The connectivity matrices were calculated for all segments segment using the correlation based on the probability of recurrence method as presented in chapter 3.2. Using this method, the phase synchronization between electrodes was obtained as the degree of connection. The connectivity matrices for each segment included the pairwise connectivity degree of all electrodes. Figure 6 illustrates the flow chart of the steps used for extracting the phase synchronization value.

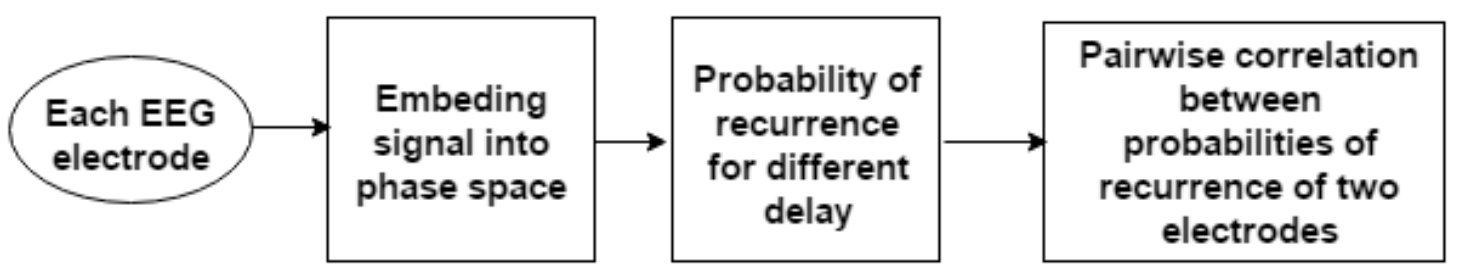

Figure 6: The block diagram of the connectivity matrices evaluation

\subsection{Results and Discussion}

Figure 7 shows the histogram diagram of a sample segment for each spike group. As it can be observed, the distribution of connections for all three groups is negatively skewed indicating a higher number of strong connections because of spike activity in the EEG segments. This characteristic was seen in all calculated connectivity maps of this study. In order to visualize the connectivity maps, connectivity matrices were depicted in head map plots showing the connections between two electrodes by a color-coded line, in which the color represented the strength of the connection from zero (blue) to one (red). 
To perform a comparative study on the functional connectivity maps in relation to the type of spikes, various thresholds were tested on the connectivity maps based on the distribution of connections. The set of $80 \%, 90 \%$ and $95 \%$, that showed the difference between the 3 groups, were selected. Figure 8, represents the head map plots for the average connectivity matrices of three biomarker groups using the selected thresholds. In Figure 8 strong highdensity activations, as a result of spike discharges, can be seen in the head map plot for all groups using a threshold level of $80 \%$. As Figure 8 shows, the distribution of connections is not uniform among all spike groups. Complex and repetitive spike maps are presenting patterns that are more symmetrical while the distribution of connections in the single spike group is not symmetrical. In order to evaluate the similarities between these 3 types of
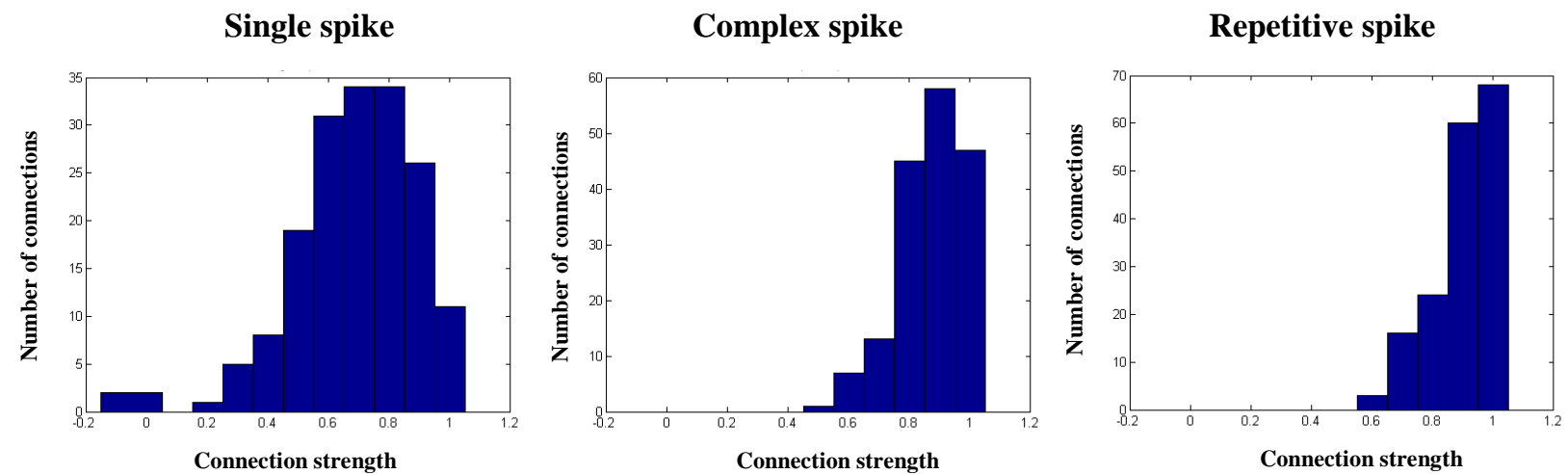

Figure 7: Histogram diagrams of a sample connectivity matrix of each spike group. 
Single Spike
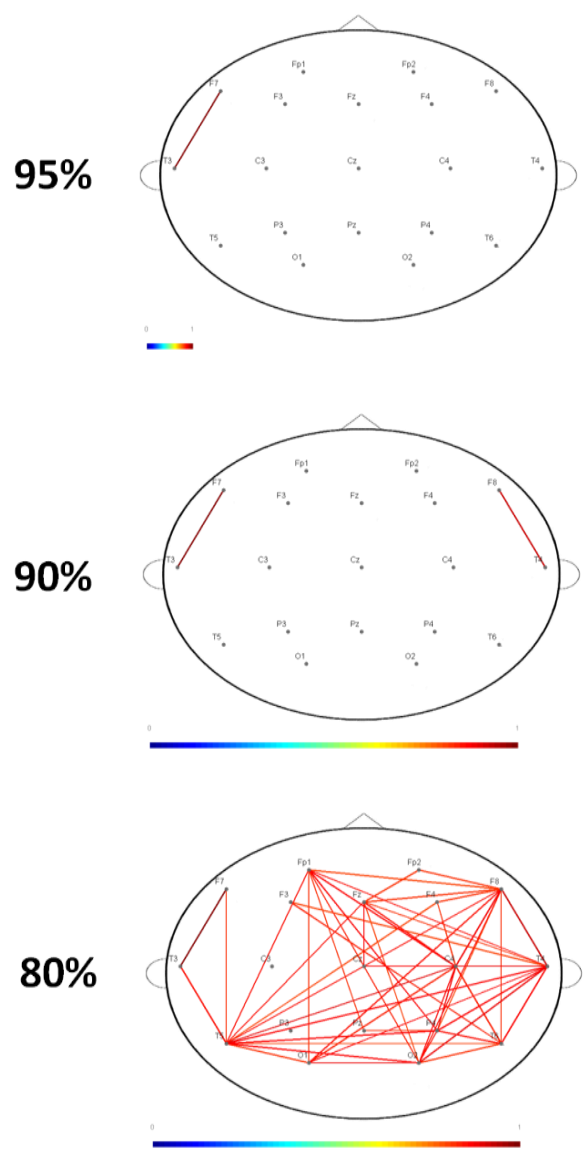

Complex Spike
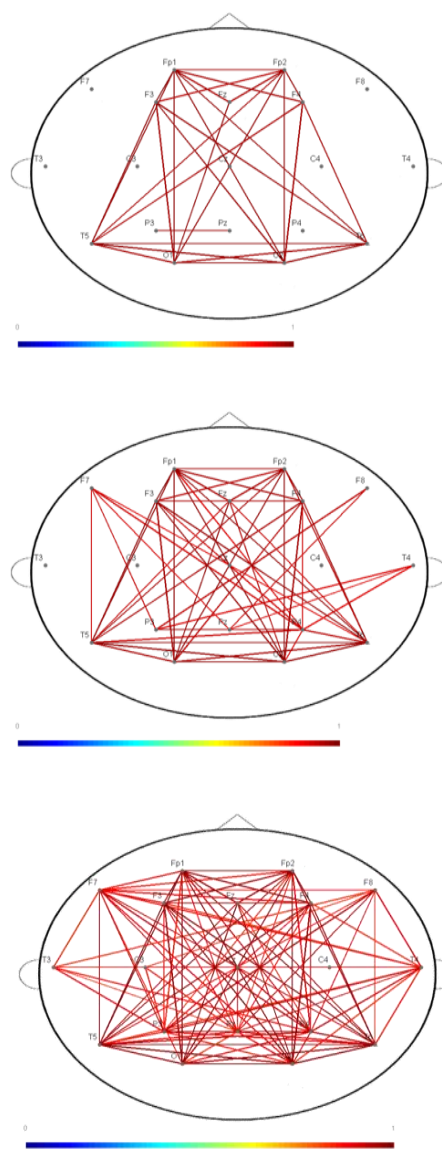

Repetitive Spike
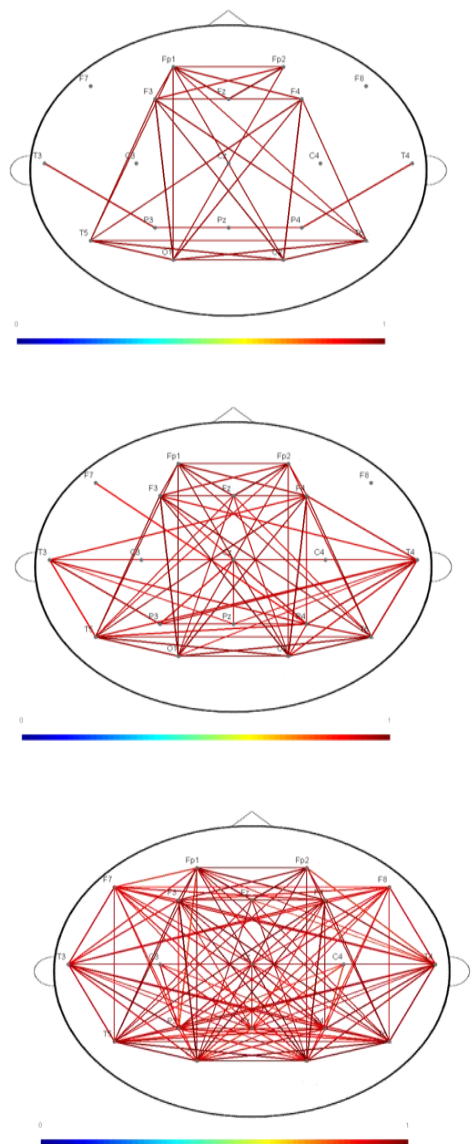

Figure 8: Connectivity maps from average connectivity matrices of the three spike groups.

spike connectivity patterns, the electrodes were grouped based on four brain regions (anterior and posterior regions and left and right hemispheres) as suggested in (Shafi et al., 2014). Anterior electrodes included Fp1, Fp2, F7, F3, Fz, F4 and F8. Posterior electrodes comprised $\mathrm{O} 1, \mathrm{O} 2, \mathrm{~T} 5, \mathrm{P} 3, \mathrm{Pz}, \mathrm{P} 4$, and $\mathrm{T} 6$ and electrodes $\mathrm{T} 3, \mathrm{C} 3 \mathrm{Cz}, \mathrm{C} 4$, and T4 were considered as border electrodes. A connection counted as an anterior connection when both connection ends were located in the anterior region, or one of the ends was a border 
electrode. Connections with both ends in the border region were ignored (Shafi et al., 2014).The same policy was considered for the posterior region, left and right hemispheres. Left hemisphere electrodes included Fp1, F3, C3, P3, O1, F7, T3 and T5; and the right hemisphere region included, Fp2, F4, C4, P4, O2, F8, T4, and T6; while Fz, Pz, and Tz are grouped as border electrodes. The number of connections for the average connectivity matrices of each group type was summarized and compared in Figure 9. The number of connections and the strength in a single spike group was lower when compared to the complex and repetitive spike groups using the same threshold. The number of interregional connections in complex and repetitive spikes was higher or close to the number of connections in each region, while this pattern cannot be observed in a single spike group, indicating more local connections, which are more indicative of focal epilepsy. Results showed that in the presence of complex and repetitive spikes, which are indicative of generalized epilepsy, all brain regions are highly connected, while in single spike cases, which are more likely to define focal epilepsy, the brain is connected locally.

The average connectivity matrices for these 3 groups of spikes were tested by analysis of variance (ANOVA) using the null hypothesis of the same mean among 3 groups. To assure the suitability of the ANOVA statistical test on the study data, the study data were checked for normality (see Figure 10). In addition, Barlett statistical test with the null hypothesis of homoscedasticity confirms that the data fits into the ANOVA assumptions (Pvalue $=0.6134)$. The ANOVA test results rejected the null hypothesis of the same mean among average connectivity groups $(\mathrm{P}$-value $\ll 0.001)$. Performing the multiple comparison studies 
Anterior-Posterior regions
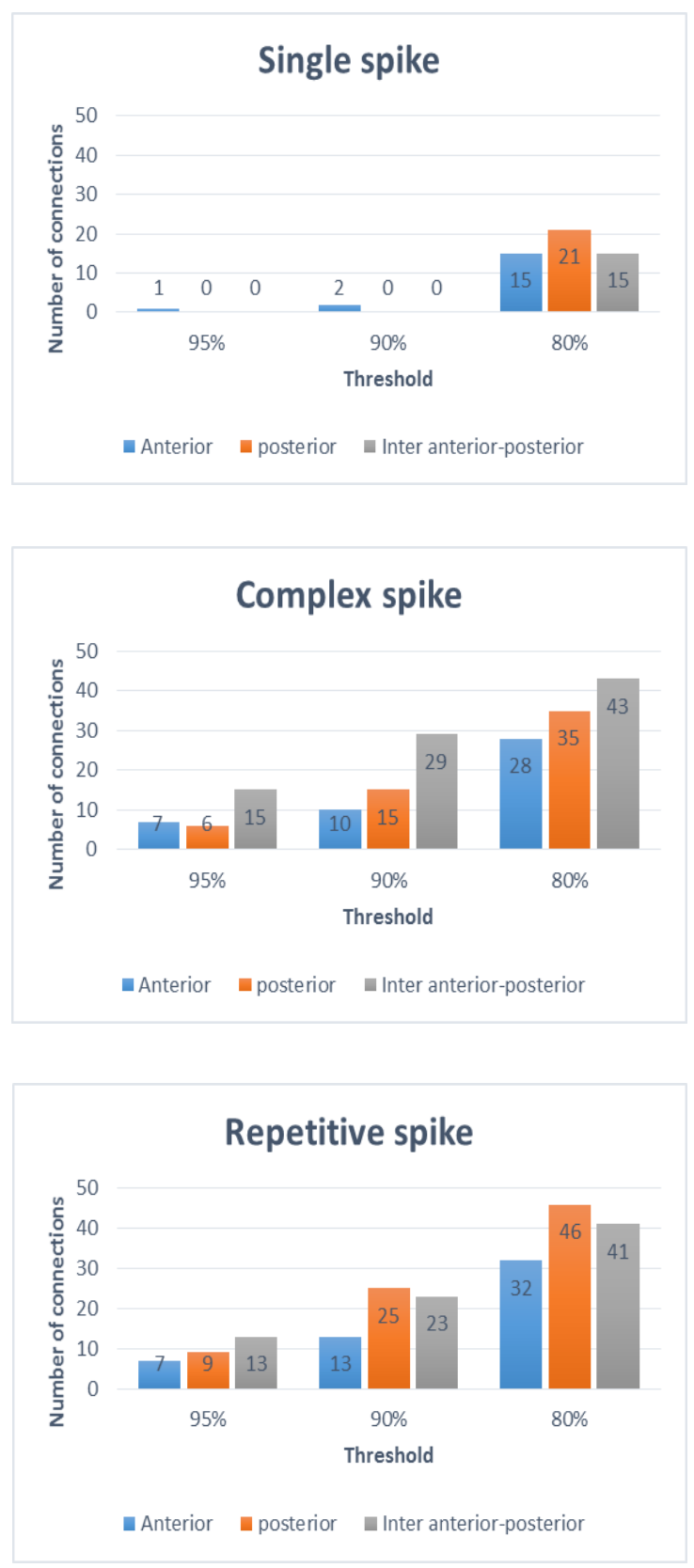

\section{Left-Right hemispheres}
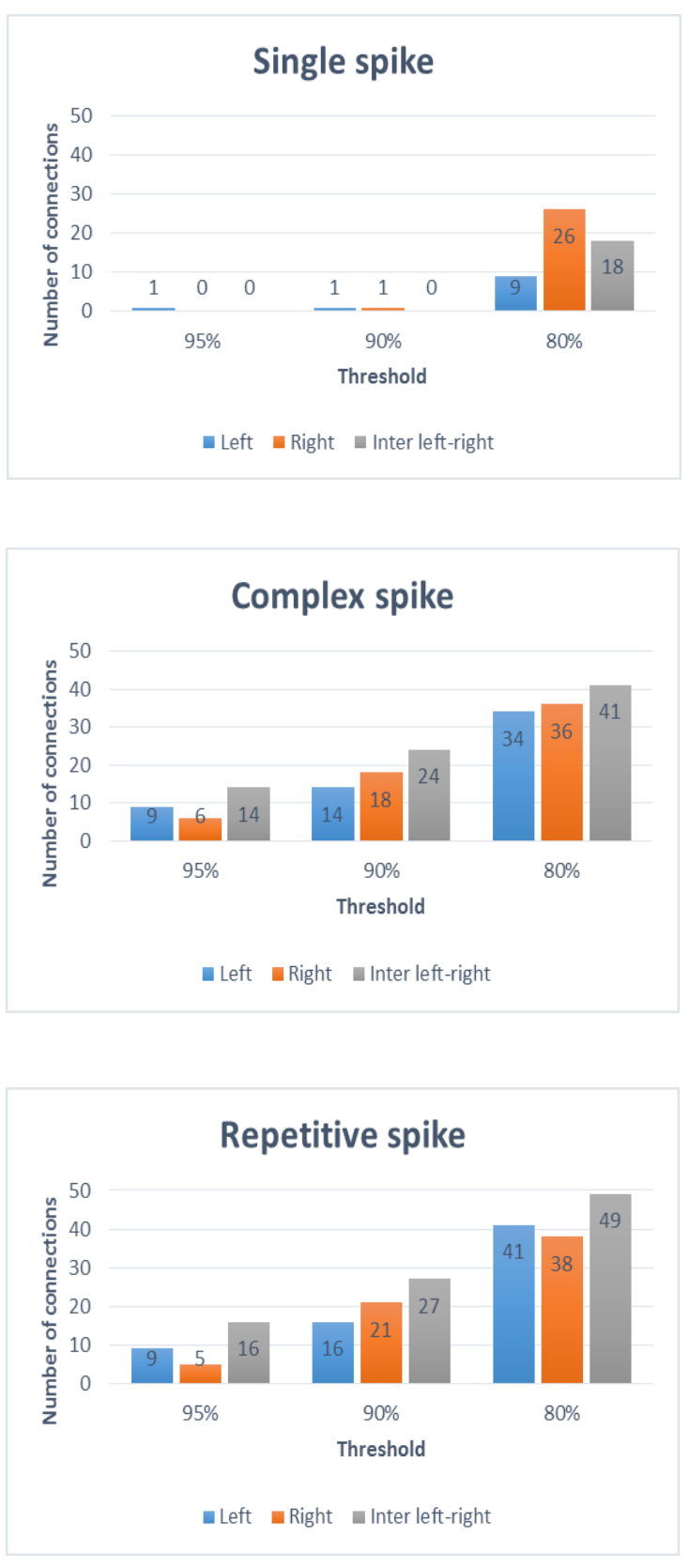

Figure 9: The quantification comparison for the main brain regions within three epileptic patterns. 


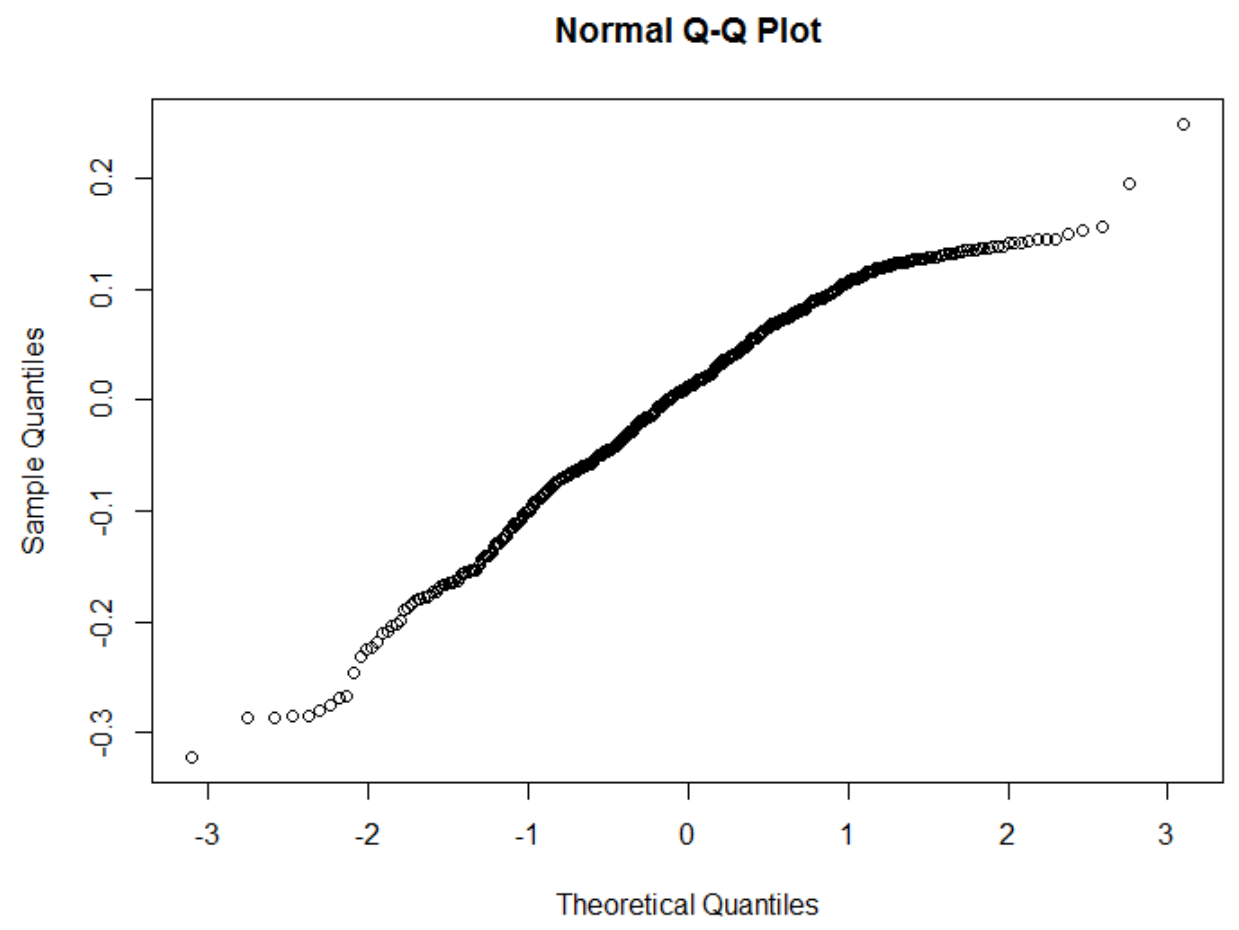

Figure 10: The Q-Q plot of the residuals of ANOVA test confirms normality of the study data.

indicated that the single spike connectivity matrix was significantly different from complex and repetitive groups as illustrated in Figure 11.

This study evaluates EEG functional connectivity maps obtained from generalized and focal epileptiform discharges to extract and investigate the functional connectivity maps using a nonlinear method. The segments were selected randomly but with same characteristics from interictal data. One of the limitations of the analysis was the variability of the results due to the background activity of the selected segments. 


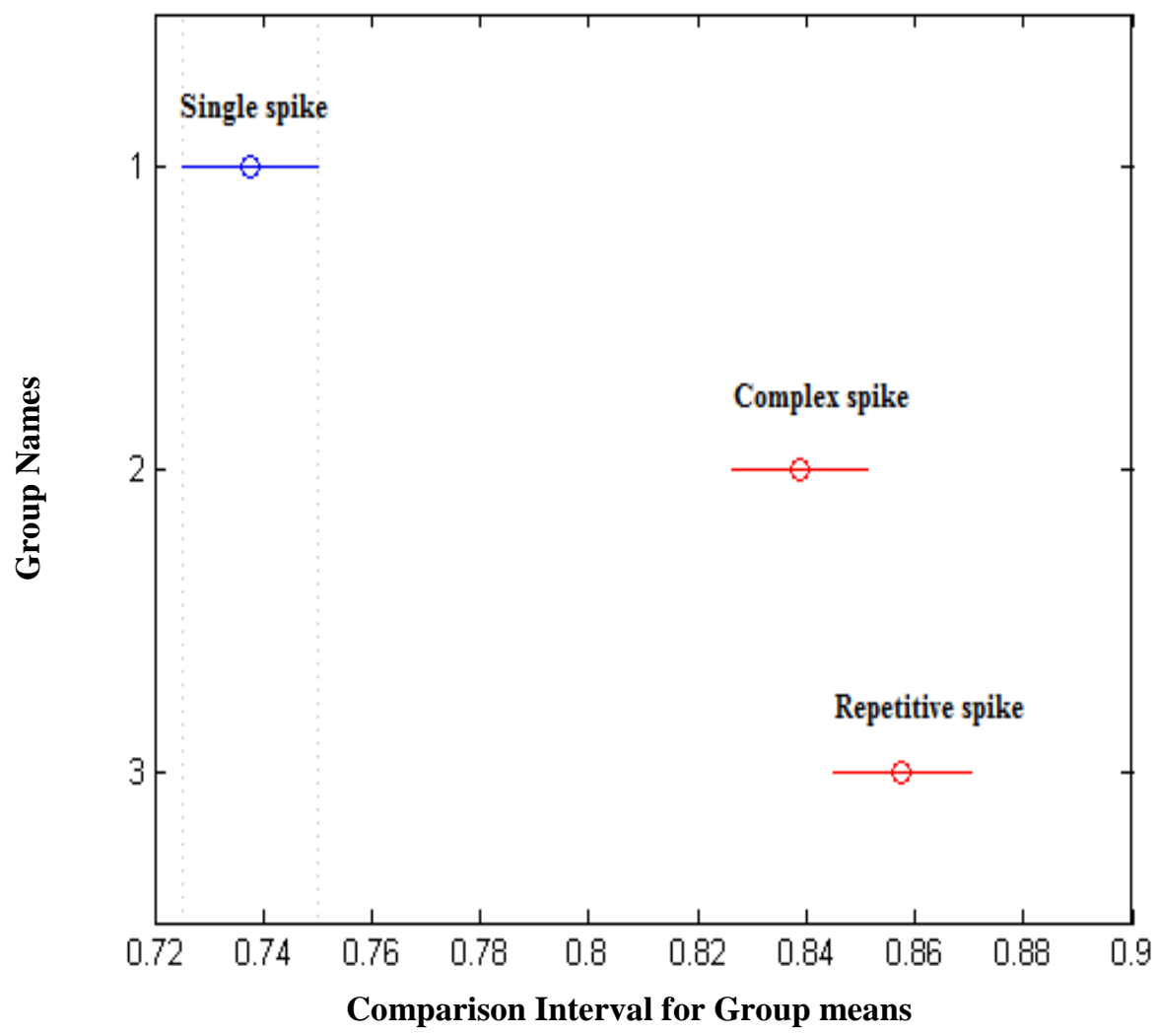

Figure 11: Multi-comparison statistic test results. The single spike group has no overlap with the complex or repetitive group indicating significant difference with respect to the other two groups.

\subsection{Conclusion}

Functional connectivity maps were used to assess EEG signals containing three different epileptic activities characterizing focal and generalized epilepsy. The connectivity patterns were extracted by a nonlinear method showing the mutual synchrony of electrodes in accordance with the 10-20 system. Various connectivity maps were investigated based on the number of connections in four major brain regions (Anterior, Posterior, Left 
hemisphere and Right hemisphere). The investigation resulted in significant differences between single spike connectivity patterns versus complex and repetitive spikes. These results support the theory that higher global connections are seen in generalized epilepsy rather than the more local connections observed in the case of focal epilepsy. The difference between connectivity patterns of these two types of epilepsy is supported by performing analysis of variance among average connectivity matrices of the three groups. The study results show that, through the use of connectivity maps, EEG signals could be used to improve the identification process of specific characteristics that delineate the three types of spikes, and consequently enhance the diagnosis for focal or generalized epilepsy solely on the basis of EEG segments that were randomly selected. 
Chapter 6

\section{CONNECTIVITY DYNAMICS OF INTERICTAL EPILEPTIFORM ACTIVITY}

\subsection{Introduction}

Patterns of interictal epileptiform activities, such as sharp waves, spikes, spike-wave complexes, and polyspike-wave complexes are explored in the recorded electroencephalograms (EEG) to gauge the different functional connectivity dynamics and to assess how they could be affected by the type of a seizure. Connectivity measures were represented by the phase synchronization among scalp electrodes that were obtained by adopting a nonlinear data-driven method. These interictal epileptic activities were investigated using a graph theory analysis. The connectivity maps were compared by considering the number of connections in four main brain regions (anterior region, posterior region, left hemisphere and right hemisphere). Results revealed interesting and different network topology for the connectivity maps. Besides, a relationship between the connectivity patterns of the recorded epileptic activities and the types of seizures was observed. This relationship was statistically confirmed by analysis of variance (ANOVA) that denoted a significant difference among connectivity patterns of sharp waves and spike activities, which were seen in focal epilepsy, in contrast to the spike-wave and polyspikewave complexes that were associated with generalized epilepsy (P-value $\cong 0)$. These results augment the prospects for diagnosis and enhance the recognition of the disease type via EEG-based connectivity maps. 


\subsection{Material}

The scalp EEG signals of four adult individuals, diagnosed as epileptic, were collected. Data was recorded at Baptist Hospital of Miami. Recorded EEG data included nineteen electrodes (Fp1, F7, T3, T5, O1, F3, C3, P3, Fz, Cz, Pz, Fp2, F8, T4, T6, O2, F4, C4, and P4) based on 10-20 international system. EEG signals were digitized by a sampling frequency of $200 \mathrm{~Hz}$. The study process was approved by the Institutional Review Board of Florida International University (protocol number: IRB-150247). EEG data included both ictal and interictal events, but only interictal parts were utilized in this study. Table 4 represents the demographic information of participants. 43 files that contained different spike types were separated and used in this research.

Table 4: Demographic Information of Participants

\begin{tabular}{cccc}
\hline Patient & Gender & Type of spike & Diagnosis \\
\hline $\boldsymbol{P 1}$ & F & Spike & Focal epilepsy (Left hemisphere-LH) \\
\hline $\boldsymbol{P 2}$ & F & Spike & Focal epilepsy(Right hemisphere-RH) \\
\hline $\boldsymbol{P 3}$ & F & Complex & Generalized epilepsy \\
\hline $\boldsymbol{P 4}$ & M & Complex \& Repetitive & Generalized epilepsy \\
\hline \hline
\end{tabular}

\subsection{Preprocessing and Artifact Rejection}

We preprocessed data before segmentation to maximize brain-related activities rather than unwanted noise. All EEG signals were processed with a 4th order Butterworth bandpass 
filter with a passing frequency range of $[0.536] \mathrm{Hz}$. The band-pass filter with zero phase characteristics was applied to eliminate the distortion effect of the filter on signals (Rajaei et al., 2015). We applied the $36 \mathrm{~Hz}$ as high cut-off frequency since the role of brain activities higher than $36 \mathrm{~Hz}$ was minimal in the final connectivity maps and the epileptiform discharge was thus emphasized. Signal baselines were also removed for all EEG data sets.

We re-referenced data sets to average montage to minimize the volume conduction problem (Guevara et al., 2005). The recorded data were processed using both the principal component analysis (PCA) and independent component analysis (ICA) to remove various artifact contaminations with the use of EEGLAB software (Delorme and Makeig, 2004).

\subsection{Segmentation}

Extracted files included sharp waves, spikes, spike-wave complexes (complex spike) and polyspike-wave complexes (repetitive spikes). We considered sharp waves and spikes in one category and referred to them as spikes in this manuscript. Therefore, three major groups of the spike, complex spike, and repetitive spike represented the different interictal epileptiform discharges in this research endeavor. The filtered, artifact-free EEG data was subdivided into one-second segments. The one-second segment length was chosen because we wanted to focus only on the dynamics of the interictal discharge, by eliminating the EEG propagation of the background activity. Figure 12 illustrates sample data segments of a spike, a complex spike, and a repetitive spike. The peak of the spike was positioned in the middle of the segment for spikes and sharp waves, while for the complex and repetitive 
(a)

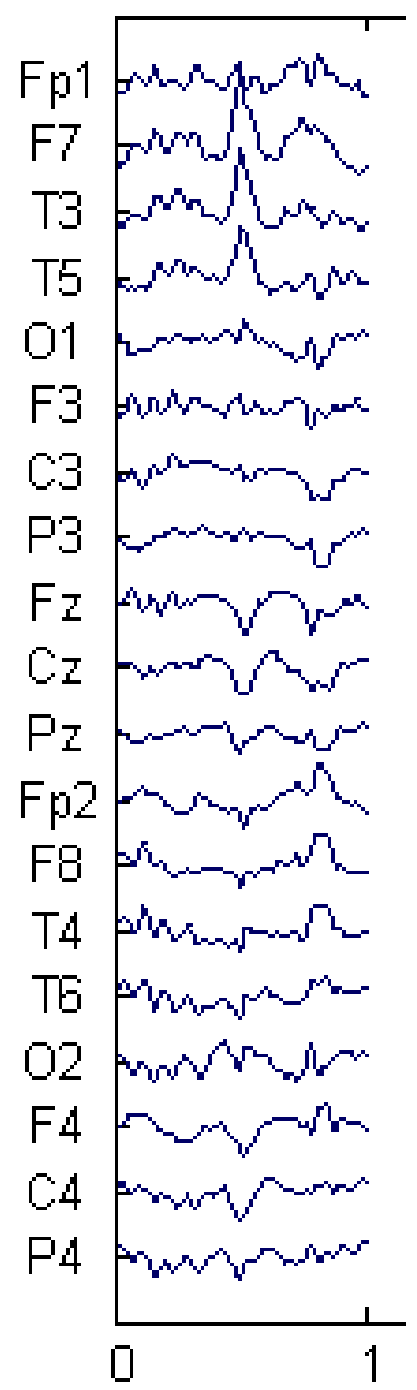

(b)

(c)

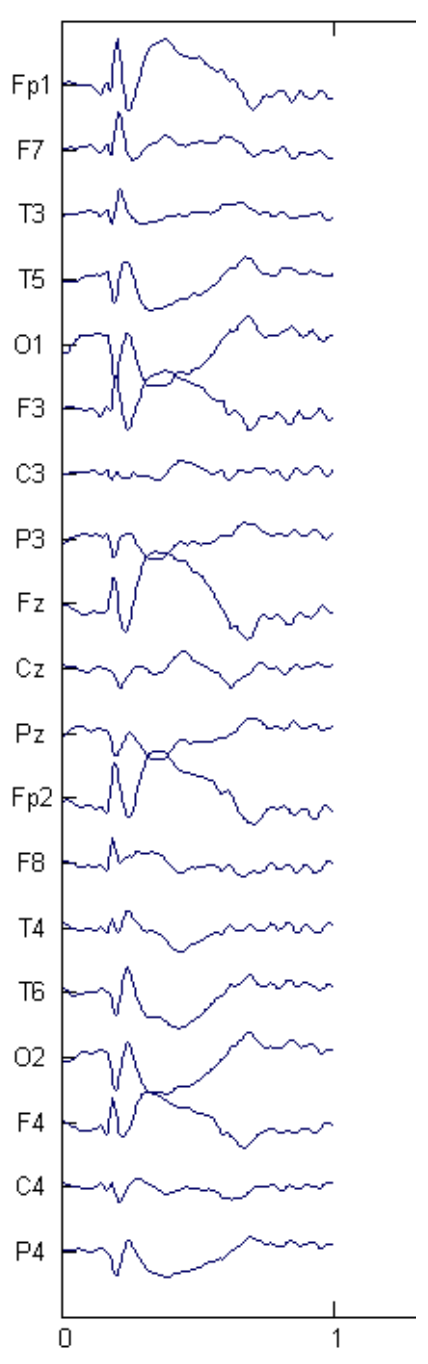

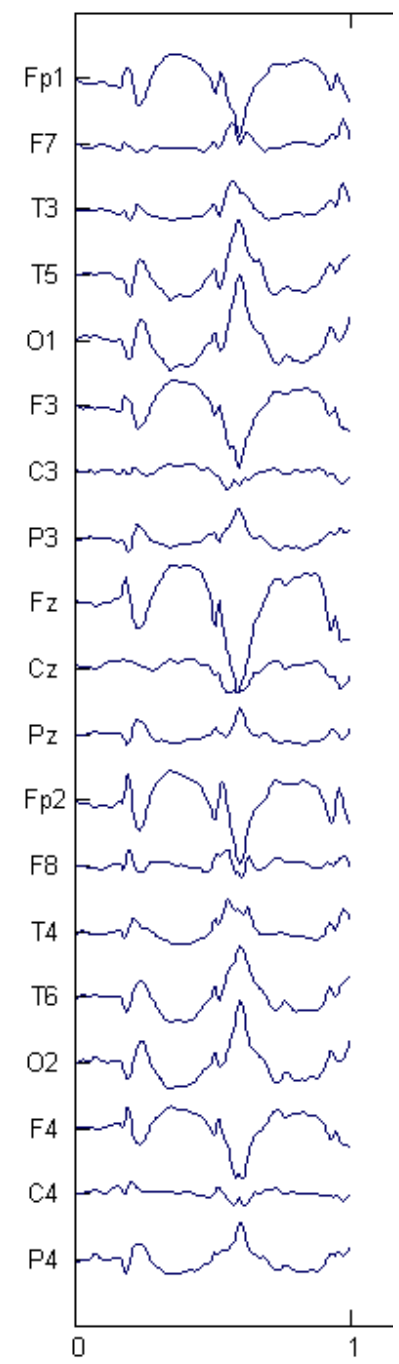

Figure 12: Samples of EEG segments used for connectivity analysis. (a) Spike (b) Spike-wave complexes (Complex spike) (c) Poly spike-wave complexes (Repetitive spike)

spikes, the peak of the first spike was placed in the first $20 \%$ portion of the one-second EEG segment (Rajaei et al., 2016). 


\subsection{Functional Connectivity maps}

Functional connectivity matrices were calculated for all the segments based on the method presented in chapter 3.2. To find a set of parameters that lead to significant results, surrogate data test (chapter 3.3) was performed for a different combination of parameter values (time lag range of [2 13] and dimension range of [3 10]). This procedure helps to find a set of parameters that lead to significant results. The parameters were chosen in a way that connectivity matrices for all segments of data be significant. Based on test results, the time lag of 3 and embedding dimension of 6 were selected for spikes and complex spikes and time lag of 2 with dimension 3 were used for repetitive spike group. For the recurrence threshold $(\varepsilon)$, the variable value of $10 \%$ of the average space diameter is calculated (Marwan et al., 2002).

\subsection{Results and Discussions}

The connectivity matrices were computed for all 43 segments, and the average matrix of the three categories (spikes, complex spikes, and repetitive spikes) was obtained. The histograms and properties of the connectivity matrices are shown in Figure 13 and Table 5. It can be observed that the connection mean value of single spikes is lower than the average values of the complex and repetitive spikes. The connection distribution for repetitive spikes represents a larger variance. All three distributions consist of powerful connections as a result of spike activity. Histograms of complex and repetitive spikes are negatively skewed, indicating a higher number of strong connections in contrast to complex 
(a)

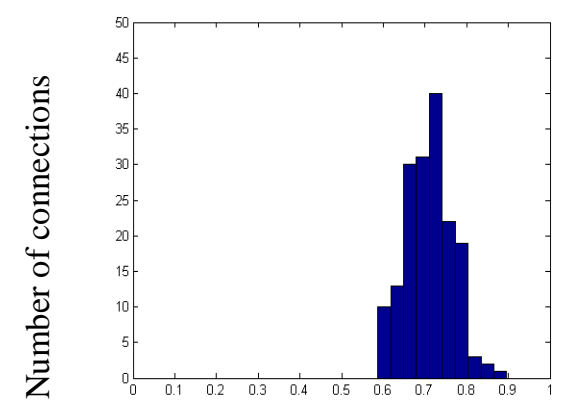

Connection strength (b)

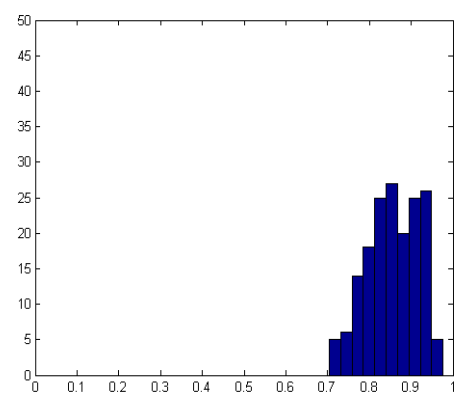

Connection strength (c)

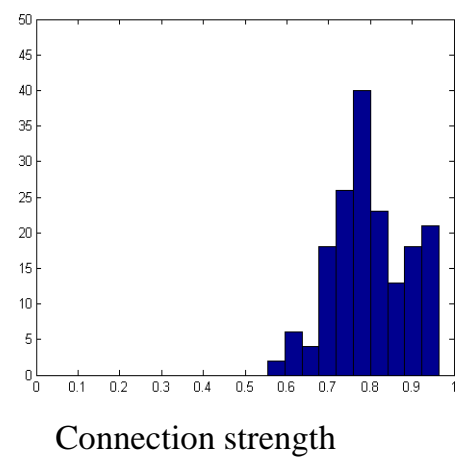

Figure 13: Histogram of average EEG connectivity maps. (a) Spike (b) Complex spike (c) Repetitive spike

and repetitive spikes. The spike connection distribution is close to a mesokurtic (Kurtosis $\cong 3$ ) distribution as compared to the complex and repetitive distributions.

Table 5. The Mean and standard deviation of average spike groups

\begin{tabular}{lcccc}
\hline IDE Category & MEAN & STANDARD DEVIATION & SKEWNESS & KURTOSIS \\
& & & & \\
\hline Spike & 0.7045 & 0.0580 & 0.1375 & 2.9772 \\
& & & & \\
\hline Complex spike & 0.8464 & 0.0587 & -0.2635 & 2.1582 \\
& & & & \\
\hline Repetitive spike & 0.7876 & 0.0941 & -0.0705 & 2.5193 \\
& & & & \\
\hline \hline
\end{tabular}

The synchronization value was obtained from all EEG connectivity matrices by averaging the value of PS calculated for all pairwise electrodes in an EEG segment (Ponten et al., 2009). In Figure 14 the synchronization values of the three spike categories are compared 


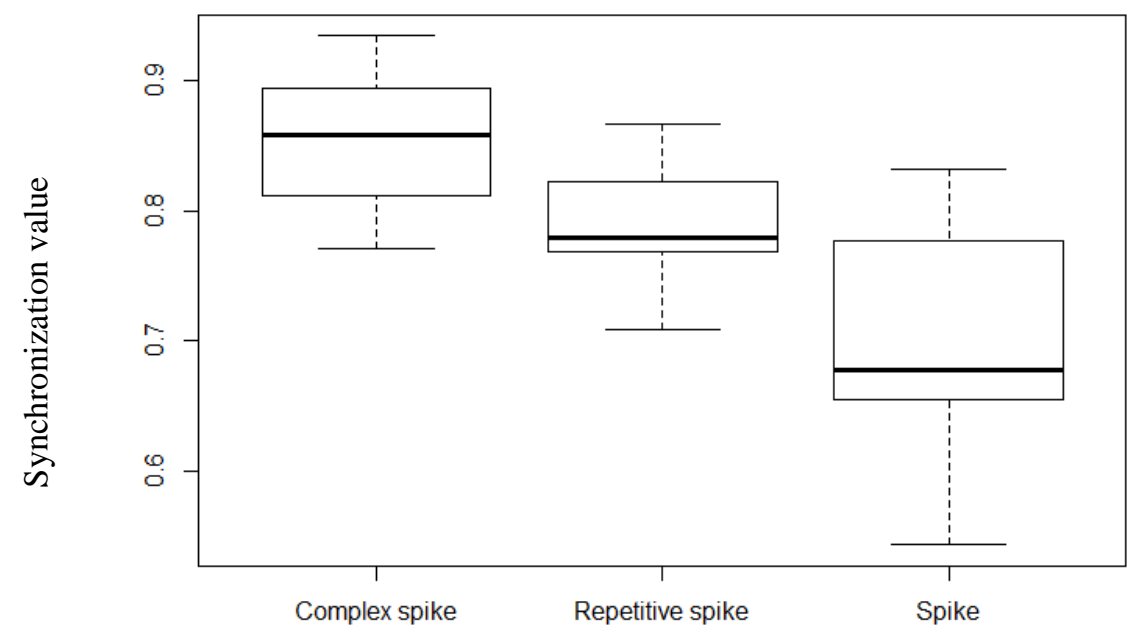

Figure 14: The comparison of synchronization value among three spike categories shows synchronization in spikes are lower than complex and repetitive spikes.

in a box plot, and higher synchronization in complex and repetitive spikes comparing to single spikes are observed.

To statistically evaluate the connectivity differences between three epileptic waveforms, the average matrices were tested by analysis of variance (ANOVA) with the null hypothesis of same means. The ANOVA test resulted in a rejection of the null hypothesis with $99 \%$ confidence $(\mathrm{P}$-value $=2.05 \mathrm{e}-56)$. Analysis of the results with the multi-comparison test showed that single spikes are significantly different from the other two categories of spikes as shown in Figure 15.

Several brain network topologies have been investigated in previous studies using the graph theory (Wilke et al., 2011; Stam and Reijneveld, 2007; Stam, 2014). It is known that 


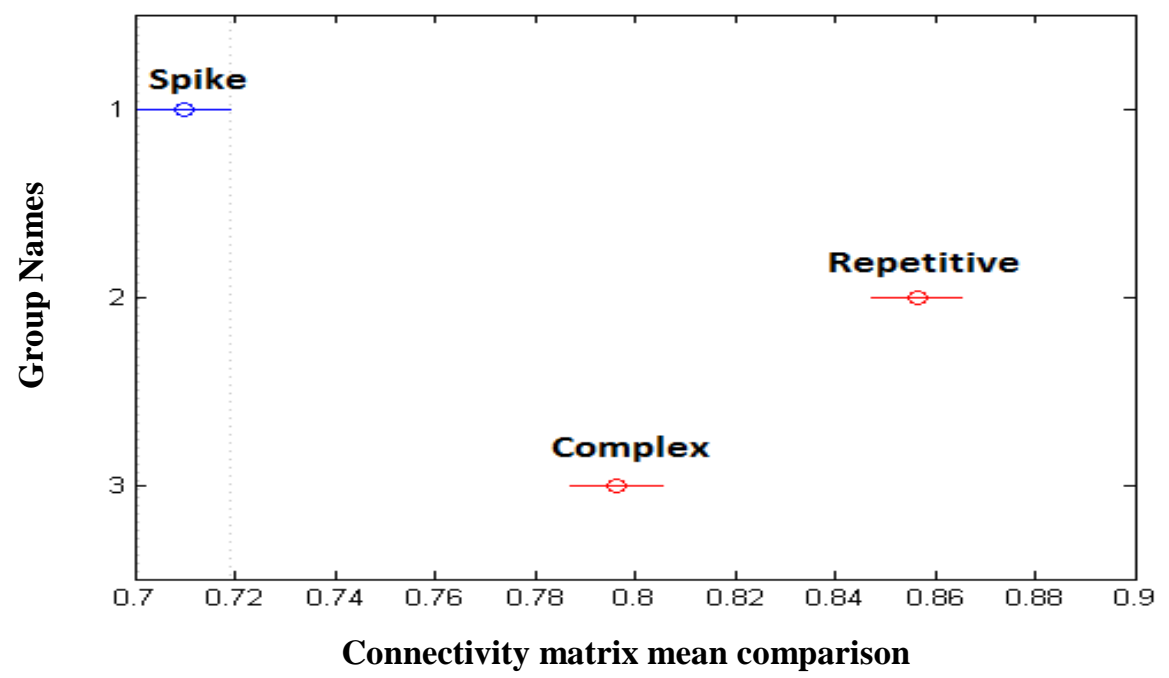

Figure 15: Multi Comparison test results shows that average connections of spikes are significantly different from complex and repetitive spikes

a healthy brain network shows the characteristics of small-world topology, which is an optimized tradeoff between integrated information processing and global communication (Stam, 2014). Here, we have considered the connectivity matrices as a weighted undirected adjacency matrix of a graph in which nodes are electrodes, and edges represent connectivity strength links between the two related electrodes (Stam, 2014). As each electrode represents the brain region underneath, the graph is showing the connectivity relation between brain modules. The average clustering coefficient $(C)$ of all vertices that is a local connectivity feature was calculated. The global communication attribute was presented by the average shortest path length $(L)$ (Ponten et al., 2009). A network with small-world topology has a high clustering coefficient with a short average path length. The ratio of $C / C_{r}$ and $L / L_{r}$ is calculated to access these attributes in connectivity graphs. 
In which $C_{r}$ and $L_{r}$ are the clustering coefficient and the average path length of the randomized initial network. The small world index is calculated as (7) (Ponten et al., 2009).

$$
S=\frac{C / C_{r}}{L / L_{r}}
$$

Figure 16 and Figure 17 shows the clustering coefficient, average path length and the small world index of categorized EEG connectivity graphs. As results indicated in these two figures, the network topology of the three spike categories is close to a random network. These results show that the small-world characteristic is lost due to interictal abnormal activity. The same result has been obtained previously because of seizure activity in the EEG signal (Ponten et al., 2009). In this study, we used the MIT MATLAB network routine toolbox for the calculation of $L$ and $C$. The small world index was coded using the MATLAB software (Bounova, 2014).

To better visualize the calculated connections, the connectivity matrices are plotted concerning electrode placements on the head while the strength of links is coded by colors. The red color indicated a strong relationship while blue indicates weaker bonding values. We applied a threshold set of $[95 \%$ 90\% 80\%] to connectivity maps to make the comparison among the interictal epileptiform activities. A sample connectivity pattern of the spike, complex and repetitive is illustrated in Figure 18.

By visual investigation of the head map plots in Figure 18, it is noted that the connection patterns are different among various interictal epileptic discharges, reflecting the spread of 


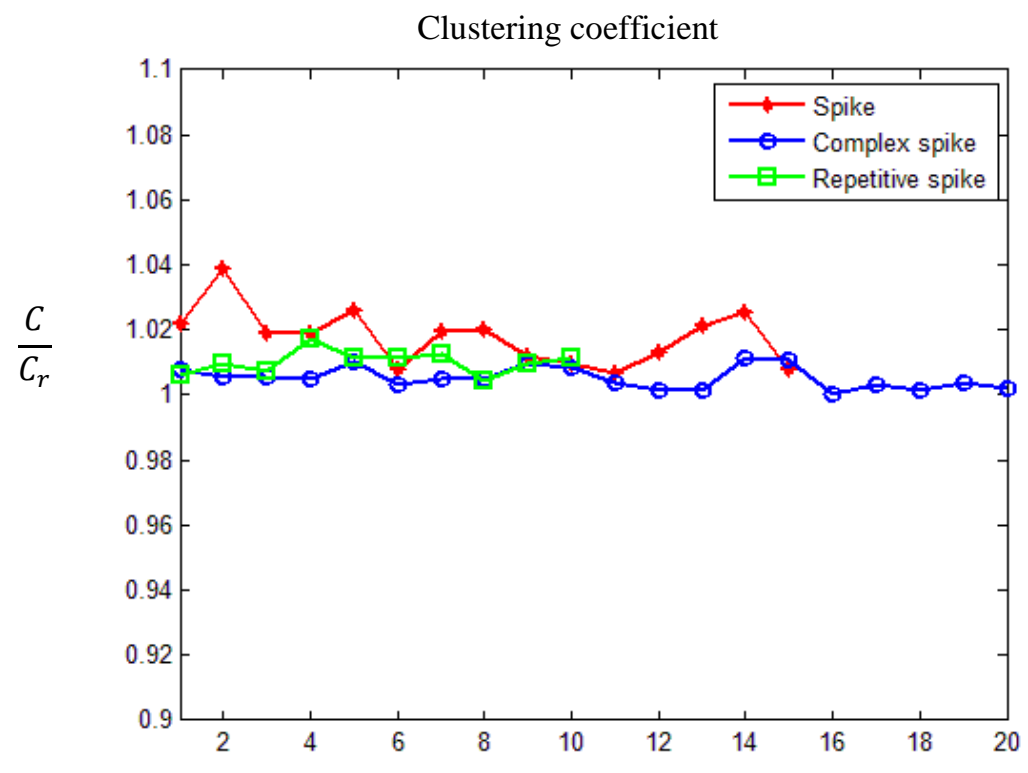

Segment index

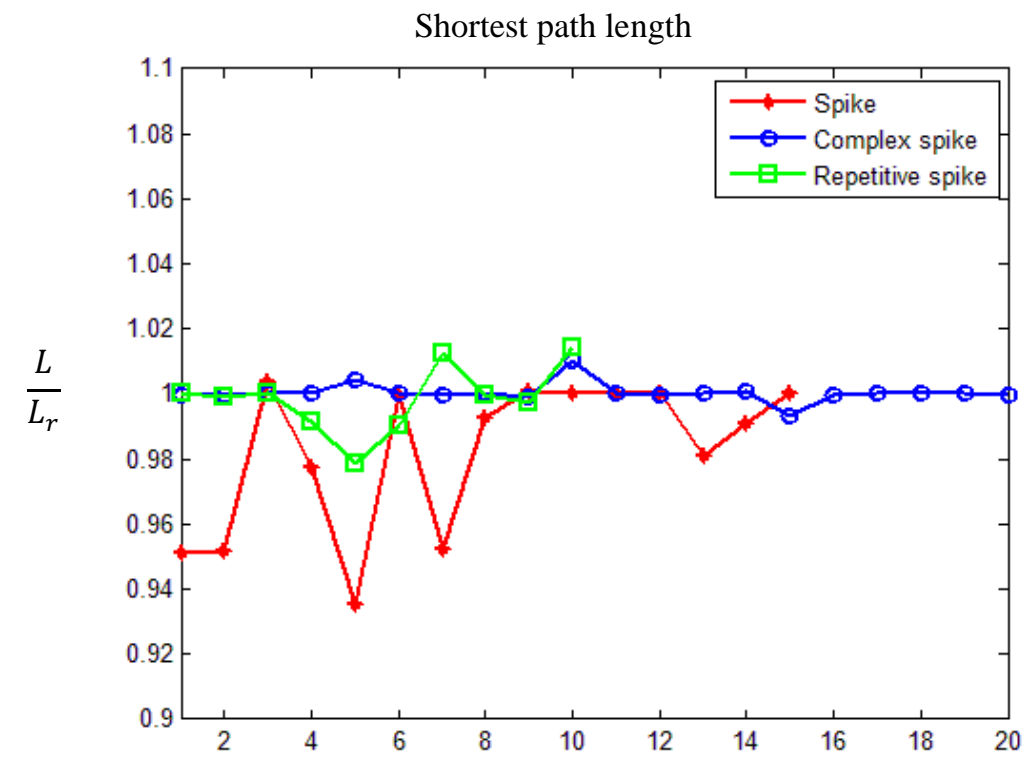

Segment index

Figure 16: The clustering coefficient (top), shortest path length (down) of the EEG connectivity maps for the three spike categories. 


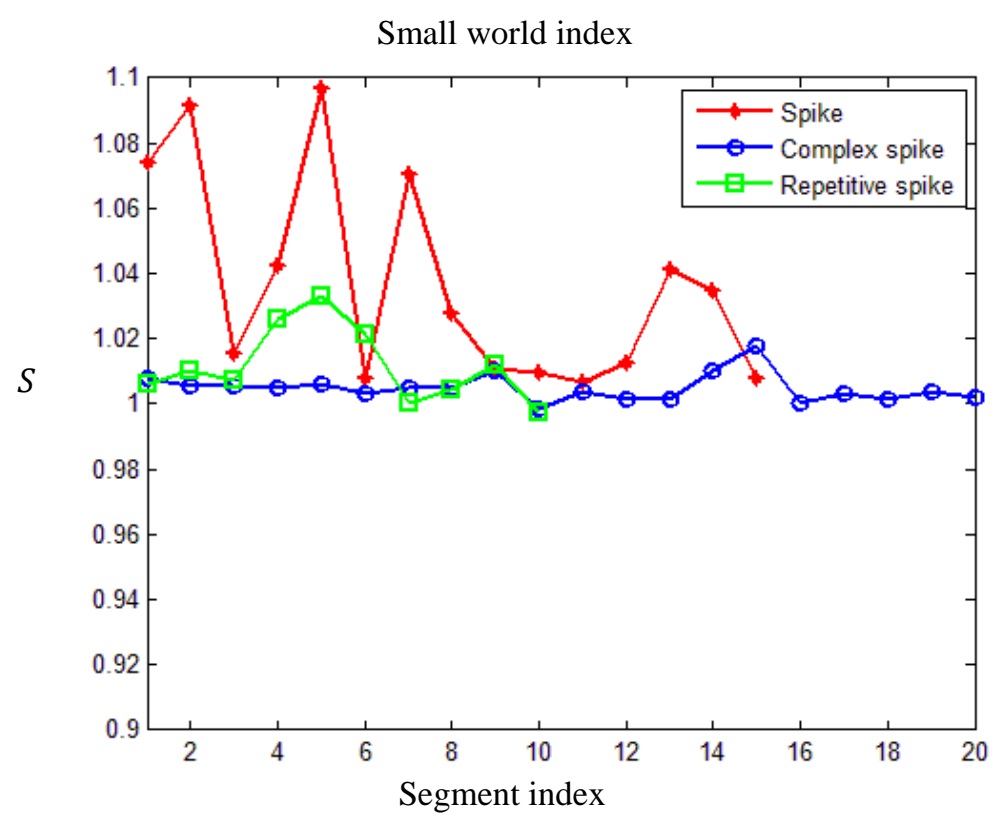

Figure 17: The small world index which shows that the epileptic interictal activities change the connectivity graph topology toward a random graph.

spike activity in the brain. Since complex and repetitive spikes are globally synchronized events, the connections appeared in most of the brain regions. This global activation creates strong relationships among the majority of electrodes in the connectivity maps.

In contrast, the single spike activity, which was seen as sudden discharges in only some of the electrodes in EEG data, resulted in weaker and more localized connections in the connectivity maps.

Four main brain regions were considered to highlight the variety of connectivity maps quantitatively. The number of connections within and between regions is counted as an indication of activity. These four brain regions are: 

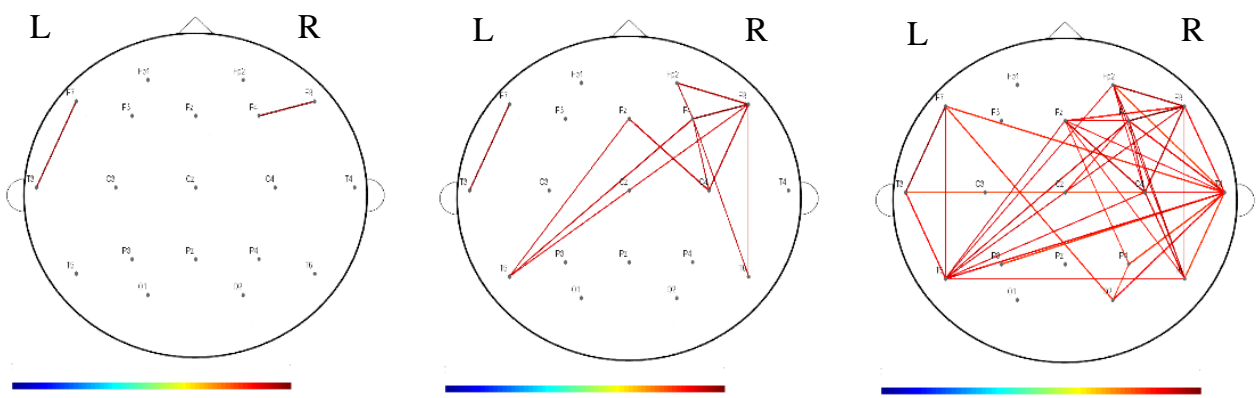

(a) Examples for single spike connectivity map (P1: Spike, focal epilepsy (left hemisphere))
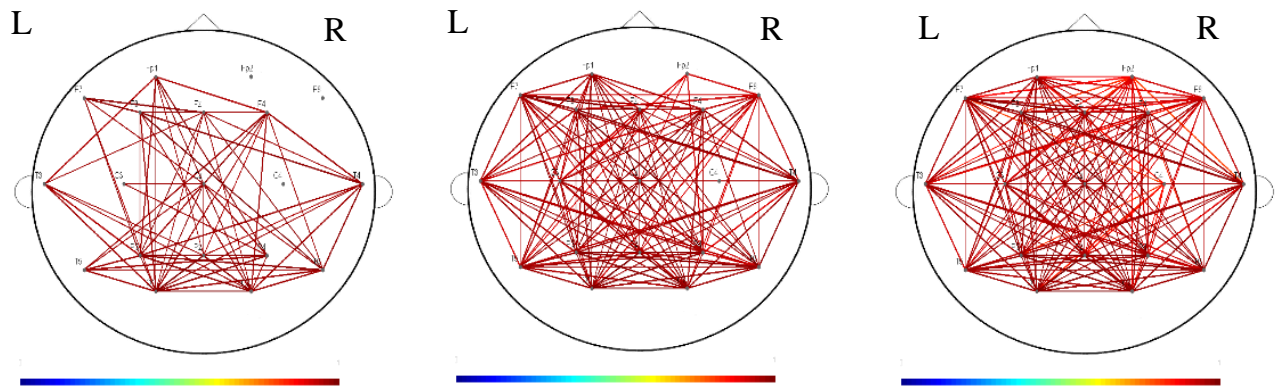

(b) Examples for complex spike connectivity map (P3: Complex spike, Generalized epilepsy)
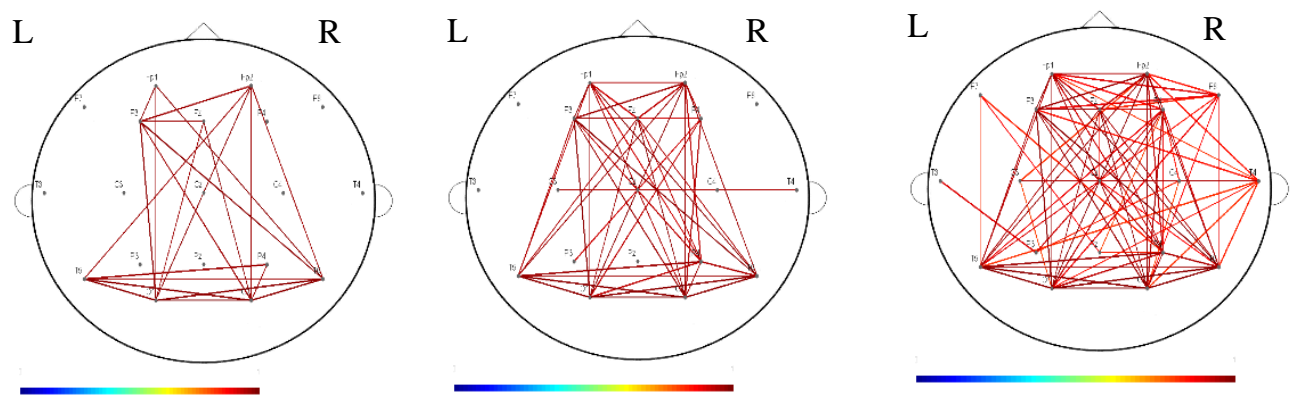

(c) Examples for repetitive spike connectivity map (P4: Repetitive spike, generalized epilepsy)

Figure 18: Head map connectivity plots of various epileptiform discharges extracted from EEG segments containing: a) Single spike b) Complex spike c) Repetitive spike 
- Anterior region (including electrodes: Fp1, Fp2, F7, F3, Fz, F4, and F8)

- Posterior region (comprised of O1, O2, T5, P3, Pz, P4, and T6)

- Left hemisphere (electrodes: Fp1, F3, C3, P3, O1, F7, T3, and T5)

- Right hemisphere (Fp2, F4, C4, P4, O2, F8, T4, and T6).

The border electrodes for the anterior/posterior are $\mathrm{T} 3, \mathrm{C} 3 \mathrm{Cz}, \mathrm{C} 4$, and $\mathrm{T} 4$; and for the left and right hemisphere are $\mathrm{Fz}, \mathrm{Pz}$, and $\mathrm{Cz}$. The within-region connections are located between two electrodes in the same region or one electrode in the area and the other one as a border electrode. Connections between border electrodes were ignored.

For each EEG segment, the counted links are compared with threshold levels of 95\%, 90\%, and $80 \%$. Figure 19 shows the comparison graph of the counted connections for the average connectivity map of various categories. In Figure 19, it is observed that complex and repetitive spikes, which are more generalized activities, have a higher quantity of betweenregion connections in all threshold levels, while this same pattern is not seen in the single spike group. In fact, a significant amount of between-region connections is a sign of global synchronization activity that characterizes mostly the generalized epilepsy cases. Hence in focal epilepsy with local discharges, the focal patterns of abnormal activity as reflected in the connectivity maps shows a higher number of within-region connections and a lower number of between-region activities. 

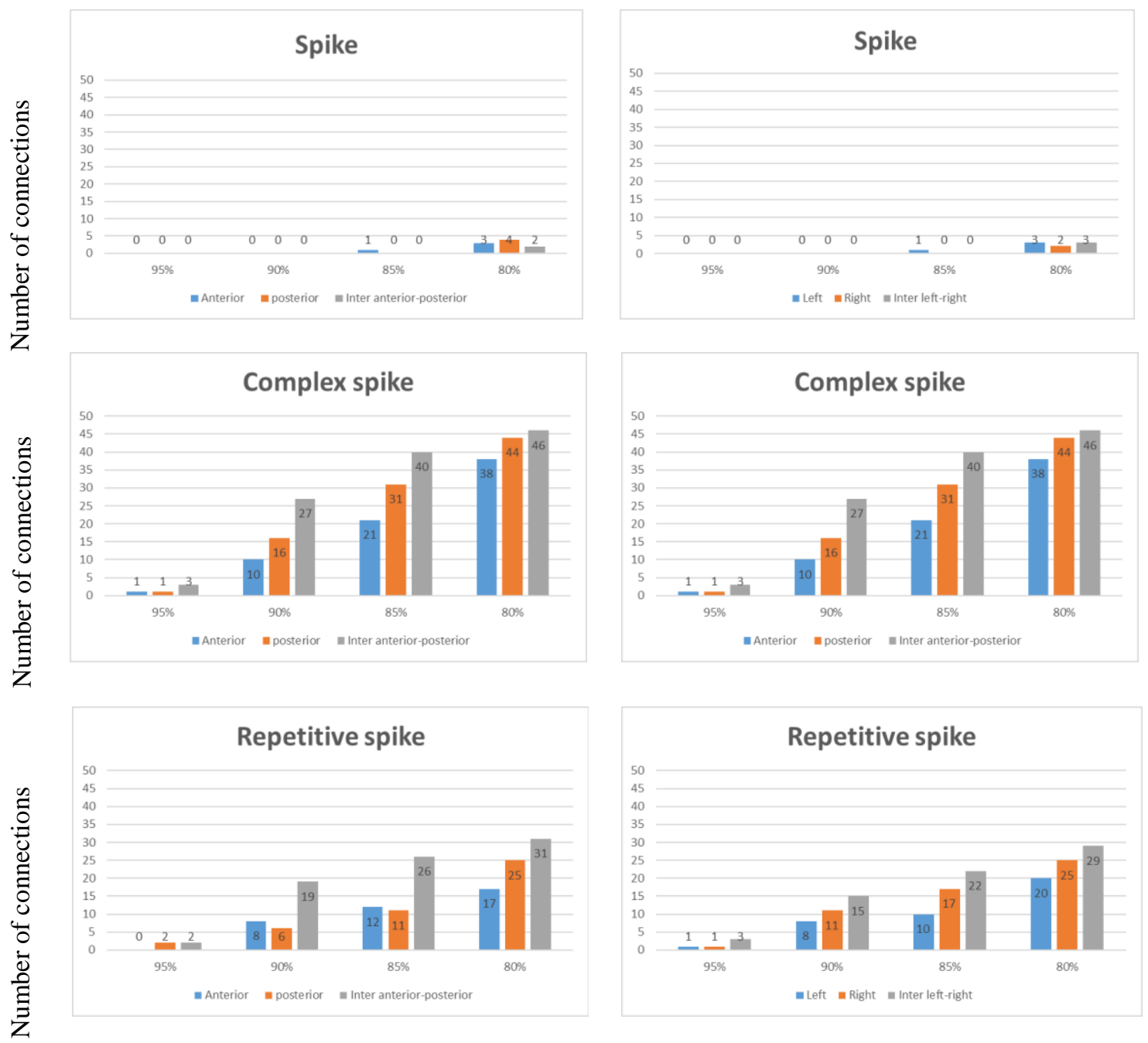

Figure 19: The quantification of average connectivity matrices in four main brain regions (anterior/posterior regions and left/right hemispheres). Each graph shows number of connections (vertical axis) in various threshold levels (Horizontal axis).

\subsection{Conclusion}

In this study, functional connectivity patterns of three primary interictal epileptic discharges were explored and characterized in direct relation to the patient's seizure type. 
A set of 43 spikes, as recorded in the EEG signals from the four individuals, diagnosed with epilepsy, were collected and the phase synchronization among electrodes was calculated using the nonlinear recurrent-based method. The graph theory analysis of connectivity networks resulted in higher synchronization in addition to the abnormal regularized network topology during these epileptic activities. The connectivity maps were quantified by the number of connections within and between the four main brain regions mentioned earlier. The activities in these areas resulted in different connectivity patterns as a consequence of the various epileptic discharges. This difference was confirmed statistically by performing the ANOVA test among the average connectivity maps of the three types of spikes investigated. The null hypothesis of the connectivity means was rejected (P-value $\cong 0)$, indicating significant differences between connectivity maps related to focal epilepsy (spike group) and generalized epilepsy (complex and repetitive group). We conclude that the connectivity patterns (the connection strength and pattern of spread on brain regions) were related to the type of epilepsy. These results could be used to augment our understanding of seizures and to enhance the automatic diagnosis of the types of epilepsy by randomly selected EEG segments. Thorough scrutiny of these activity patterns in the connectivity maps could elicit new ways at determining a more accurate $3 \mathrm{D}$ source localization of seizure onsets. This last assertion is part of the research we are currently conducting with a newly approved study with Baptist Hospital. 
Chapter 7

\section{DYNAMICS AND DISTANT EFFECTS OF TEMPORAL EPILEPTOGENIC FOCUS USING FUNCTIONAL CONNECTIVITY MAPS}

\subsection{Introduction}

Objective: Patterns of interictal epileptiform discharges (IED) are all subtle indicators of where the 3D source of a seizure may be located. These specific patterns are explored in the recorded electroencephalograms (EEG) of 10 individuals diagnosed with temporal lobe epilepsy to assess how their functional brain connectivity maps could be affected by the 3D onset of a seizure. Methods: Functional connectivity maps, estimated by phase synchrony among EEG electrodes, were obtained by applying a data-driven recurrencebased method. This is augmented through a novel approach for selecting optimal parameters that produce significant connectivity matrices that are deemed essential for assessing epileptiform activity in relation to the 3D source of the seizure onset. These functional connectivity matrices were evaluated in different brain areas to gauge the regional effects of the 3D epileptic source. Results: Empirical evaluations indicate high synchronization in the temporal and frontal area of the effected epileptic hemisphere, while strong links connect the irritated area to frontal and temporal lobes of the opposite hemisphere. Conclusion: Epileptic activity which originates in the temporal area affects the temporal and frontal areas in both hemispheres. Significance: The results as obtained 
can elicit a new understanding of the dynamics of temporal lobe epilepsy in context to the epileptogenic zone and the affected distant areas of the brain.

\subsection{Data}

In this study, we have collected the scalp EEG recordings of ten adult individuals. All participants were diagnosed with Temporal Lobe Epilepsy (TLE) and were assessed for locating the position of epileptic foci by a neurologist. Nineteen electrode EEG signals, including Fp1, F7, T3, T5, O1, F3, C3, P3, Fz, Cz, Pz, Fp2, F8, T4, T6, O2, F4, C4 and P4, were recorded based on 10-20 international placement montage and digitized using the sampling frequency of $512 \mathrm{~Hz}$. The channel $\mathrm{Cz}$ was utilized as the reference channel. The data was recorded at Miami Baptist Hospital and subjects were instructed to be relaxed and avoid movement as much as possible during the recording session. The study process was approved by the Institutional Review Board of Florida International University (protocol number: IRB-150247) and written consents were received from all individuals. EEG data included both ictal and interictal events, but only interictal parts were retained in this study. We have separated the interictal EEG data that included an epileptic discharge marked by a neurologist. Table 6 represents the demographic information of participants. 
Table 6: Demographic information of participants

\begin{tabular}{|c|c|c|c|}
\hline Individual ID & Gender & Diagnosis & Epileptic Source Region \\
\hline$P 1$ & $\mathrm{~F}$ & TLE $^{*}$ & $\mathrm{LT}^{*}$ \\
\hline$P 2$ & $\mathrm{M}$ & TLE & $\mathrm{RT}^{*}$ \\
\hline$P 3$ & $\mathrm{M}$ & TLE & RT \\
\hline$P 4$ & M & TLE & RT \\
\hline P5 & $\mathrm{F}$ & TLE & LT \\
\hline P6 & $\mathrm{F}$ & TLE & RT \\
\hline$P 7$ & $\mathrm{~F}$ & TLE & RT \\
\hline$P 8$ & M & TLE & LT \\
\hline$P 9$ & M & TLE & LT \\
\hline$P 10$ & $\mathrm{~F}$ & TLE & $\mathrm{RT}_{2} \mathrm{LFT}^{\dagger}$ \\
\hline
\end{tabular}

\subsection{Preprocessing and Artifact Rejection}

Data were preprocessed before segmentation to highlight brain-related activities and minimize the effects of unwanted noise. We applied a 4th order Butterworth band-pass filter with a passing frequency range of $[0.570] \mathrm{Hz}$. The band-pass filter was applied with zero phase to eliminate the distortion effect of the filter on signals (Rajaei et al., 2015). A notch filter with a cut-off frequency of $60 \mathrm{~Hz}$ was used to suppress the AC line noise, and the DC offset was removed from all EEG channels. 
In EEG data analysis, the problem of common sources, whether as volume conduction or active reference electrode, affects the connectivity results (Stam et al., 2007; Guevara et al., 2005). A common reference electrode causes a distortion in calculated synchrony among electrodes and results in false phase synchronization values (Majumdar, 2006). In our study, we re-referenced the data sets to average montage to overcome this problem. Average reference is normally used in connectivity analysis of EEG to solve this problem (Golshan et al., 2016). Artifact contaminations such as eye blink, jaw and muscle movements are removed from the data by applying both the principal component analysis (PCA) and independent component analysis (ICA) with the use of EEGLAB software (Delorme and Makeig, 2004).

\subsection{Segmentation}

The International Federation of Societies for Electroencephalography and Clinical Neurophysiology (IFSECN) categorizes interictal discharges into four categories of sharp waves, spikes, spike-wave complexes (complex spike) and polyspike-wave complexes (repetitive spikes) (Noachtar et al., 1999). Generally, Sharp waves and spikes are associated with partial epilepsy. Since our study population is TLE patients, we had mostly sharp waves and spikes as an interictal epileptic activity, but there were cases where a wave follows the spike. The filtered, artifact-free EEG data were subdivided into three-second segments as suggested by the neurologist as a means for such segments to be physiologically and computationally meaningful. Epileptiform waves are aligned to the 
same position in the segment in order to have an equal share of epileptiform activity in the selected segments. We positioned the wave peak in the middle for all extracted segments (Rajaei et al., 2016). Fifty segments of EEG including spike or sharp wave were separated from the recorded data. Figure 20 illustrates sample data with a spike, a spike followed by a wave and a sharp wave.

\subsection{Functional Connectivity Maps and Parameters}

The surrogate data test was performed for a different combination of parameter values (time lag range of [2 13] and dimension range of [3 10]) to find a set of parameters that lead to significant results. We had chosen the ranges to include the optimal parameters obtained via mutual information and the kth nearest neighbor methods. The final values of the parameters were chosen in a way that connectivity matrices for most of the segments be significant. Based on test results, the time lag of 3 and an embedding dimension of 6 were selected for all data. The connectivity matrices for three segments were found to be not significant with any parameter. Therefore, we exclude them from our dataset, and 47 segments are then used for connectivity assessments.

\subsection{Results}

Fifty segments of EEG data featuring interictal epileptic activity have been used for this study. Phase coupling over various brain modules represented by EEG electrodes was 
(a)

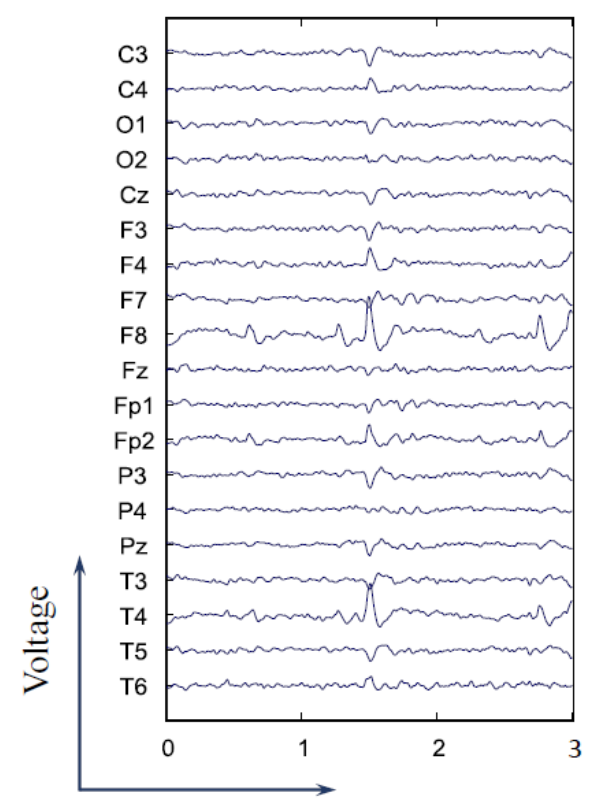

Time (s) (b)

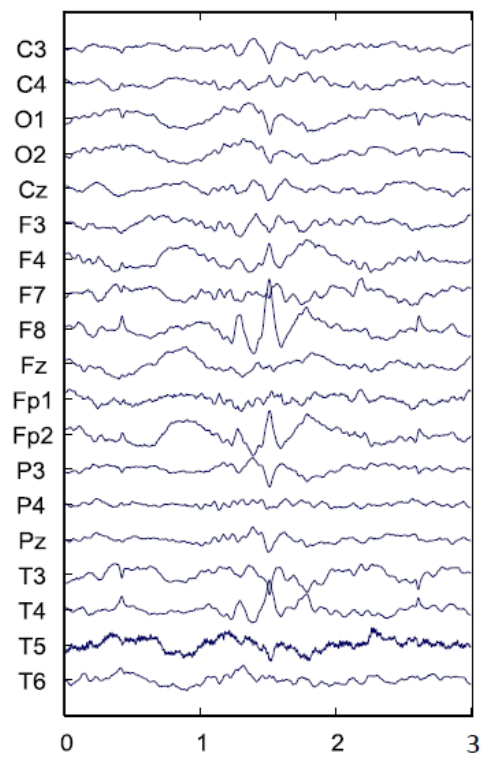

(c)

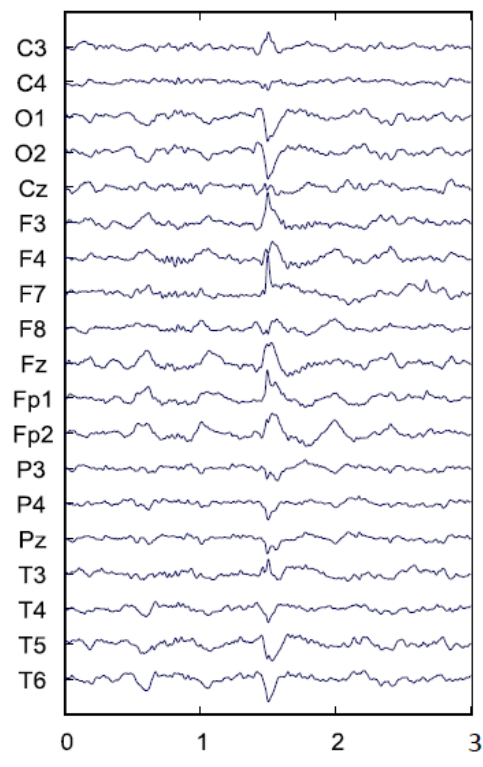

Figure 20: Sample segments of data: (a) Spike (b) Spike followed by a wave and (c) Sharp wave

obtained by applying a data-driven recurrent-based method using EEG signals in the time domain. Parameters were adjusted to yield significant functional connectivity values using the surrogate data method, and the significance of connectivity matrices was assured. From the fifty EEG segments, three have not yielded the significant connectivity matrices (three segments from $\mathrm{P} 4, \mathrm{P} 5$, and $\mathrm{P} 10$ ) for the range of parameters, and these three segments were excluded from our study. The remaining 47 matrices were considered for the assessment of the epileptogenic zone in functional connectivity maps.

We have separated the links higher than 0.75 as strong couplings that resulted from spike activity and the links below this value were ignored. We have chosen this threshold by 
investigating all the connectivity matrices. To explore connections concerning their spatial locations on the scalp, we plotted the connectivity matrices on the head map after pruning them with the threshold. The strength of connections is depicted by color codes representing dark red as a very strong connection and dark blue as a weak coupling.

To highlight the behavior of the epileptogenic zone in connectivity maps, we explored the activity of each region as local connectivity, and also we looked into the strong links that connect two local areas as distant connections. We have divided the brain cortex into six local functional zones as depicted in Figure 21, and Table 7 lists the related electrodes.

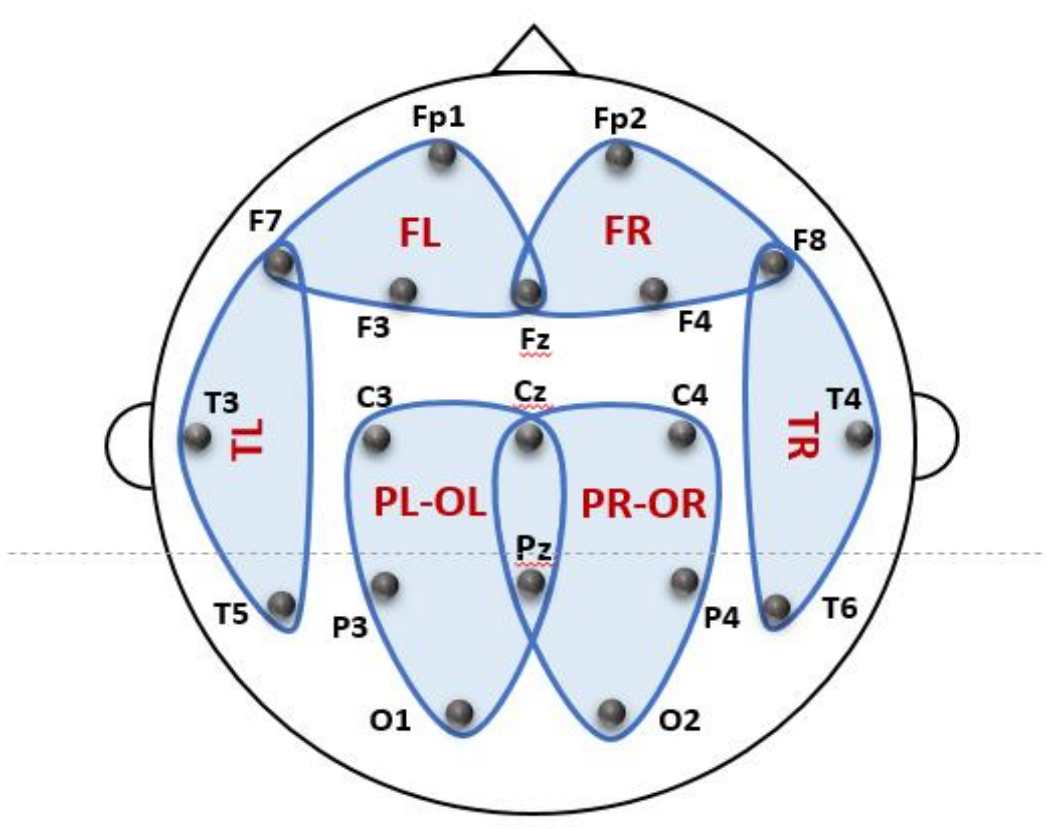

Figure 21: The different local brain regions considered: see Table 7 for additional details. 
Table 7: Six brain local regions and related electrodes

\begin{tabular}{lcc}
\hline Region name & Abbreviation & Electrodes \\
\hline Left Frontal & FL & Fp1, Fz, F3, F7 \\
\hline Right Frontal & FR & Fp2, Fz, F4, F8 \\
\hline Left Temporal & TL & F7, T3, T5 \\
\hline Right Temporal & TR & F8, T4, T6 \\
\hline Left Parietal and Left Occipital & PL-O1 & C3, Cz, P3, Pz, O1 \\
\hline Right Parietal and Right Occipital & PR-O2 & $\mathrm{C} 4, \mathrm{Cz}, \mathrm{P} 4, \mathrm{Pz}, \mathrm{O} 2$ \\
\hline \hline
\end{tabular}

We have investigated the relationship between local areas to obtain the propagation pattern resulting from the IED activity. We considered couplings between the left frontal and left temporal (FL-TL), right frontal and right temporal (FR-TR), left temporal and left parietal (TL-PL), right temporal and right parietal (TR-PR) as distant connections within one hemisphere. The distant relationships between the left and right hemispheres are also checked by calculating links between the left and right frontal regions (FL-FR) and left and right temporal regions (TL-TR). Figure 22 shows the activities in six local areas, for two cases of left and right temporal lobe epilepsy and Figure 23 represents distant connections between local regions of Figure 22 for the sample connectivity maps.

The spike activity causes higher synchrony in the brain, and as a result, more connections are going to be revealed in connectivity maps. To correlate the region's activity with the epileptogenic zone, we have quantified the activity of local regions by calculating the activity percentage for each local area. Activity percentage is obtained by dividing the 
number of available connections over the number of possible connections for that area as formulated in (7)

$$
P A_{x}=\frac{l_{x}}{L_{t}} \times 100
$$

Where $P A_{x}$ denotes the activity percentage for the region $x . l_{x}$ is the number of available connections in the region and $L_{t}$ is the number of possible connections in the relevant local region. Local brain areas are ranked based on the percentage activity to find the most active regions.

In our study population, the largest IED normally observed in electrodes that are located in the epileptogenic zone or a nearby area. However, there were three cases (P5, P7, and P9) that electrodes with largest epileptic activity could not be declared. Figure 20 (a), shows a sample data segment of P2 with clear spike activity observed in F8 and T4, and Figure 20 (c), shows a segment from P5 that the largest IED is not obvious among electrodes.

The average percentages in terms of activity over all segments of one individual are represented as bar graphs in Figure 24 and Figure 25. The study of ranked regional activity percentages indicates high activity in affected temporal lobe as expected. In addition to the temporal zone, the same side frontal area is also highly stimulated. The activity spans to the right and left frontal lobes and also the temporal lobes from another hemisphere. In some cases, one of the parietal-occipital lobes is showing the activity. Interestingly, by investigating the distant connections, it can be seen that there is an active distant link between two active regions showing the flow of epileptic activity from a source region into a distant area. 


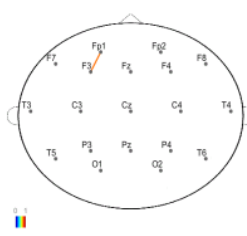

Left frontal region

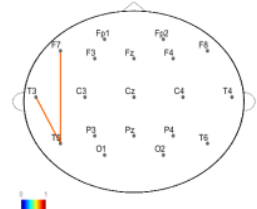

Left temporal region

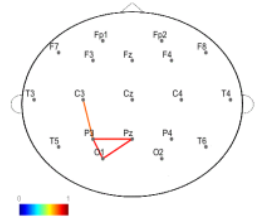

Left parietal region (a)

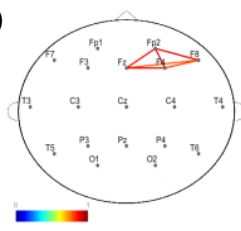

Right frontal region

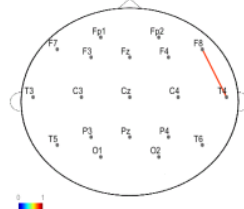

Right temporal region

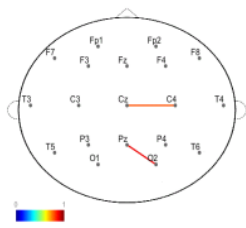

Right parietal region

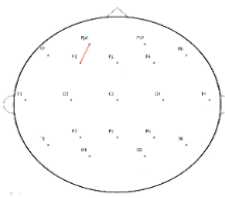

Left frontal region

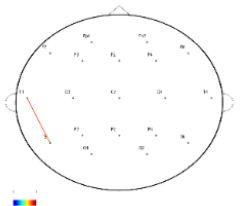

$-$

Left temporal region

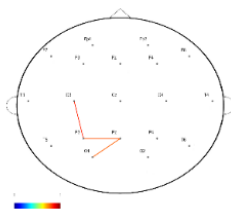

Left parietal region (b)

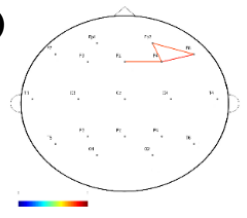

Right frontal region

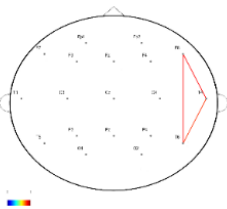

Right temporal region

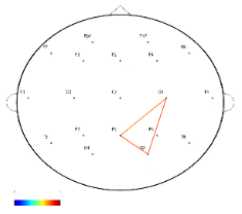

Right parietal region (c)

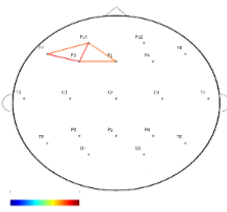

Left frontal region

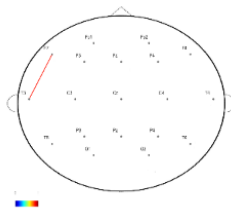

Left temporal region

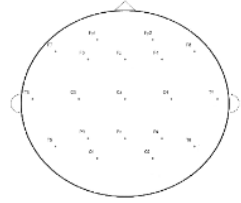

Left parietal region

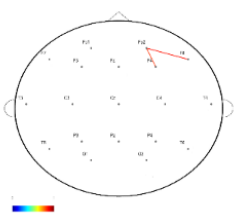

Right frontal region

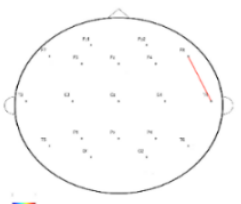

Right temporal region

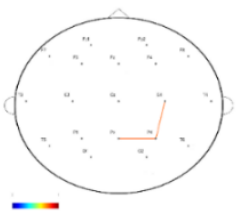

Right parietal region

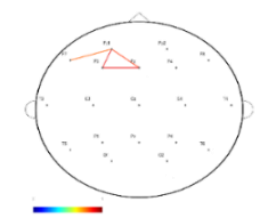

(d)

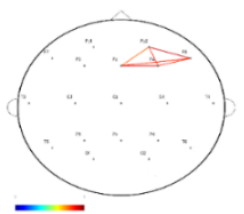

Left frontal region

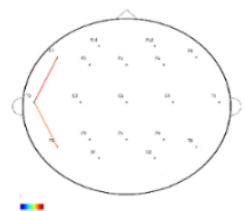

Left temporal region

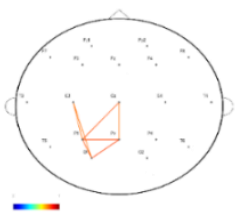

Left parietal region
Right frontal region

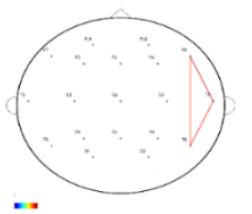

Right temporal region

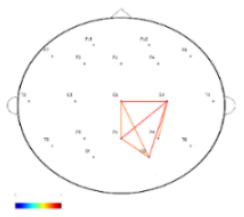

Right parietal region

Figure 22. Head map connectivity plot of six local regions resulting from a sample EEG segment of (a) P3 and (b) P4 with epileptic focus in right temporal and (c) P1 and (d) P9 with epileptic focus in left temporal area. Note: only strong connections are shown. 


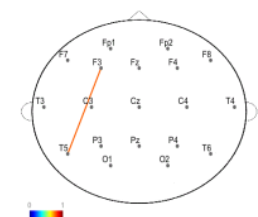

Left frontal-temporal

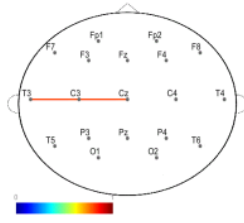

Left temporal-parietal

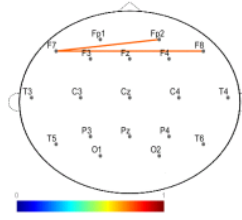

Left frontal-Right frontal

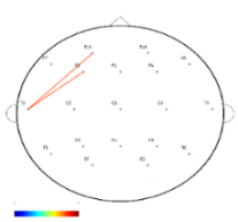

Left frontal-temporal

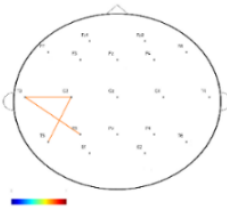

Left temporal-parietal

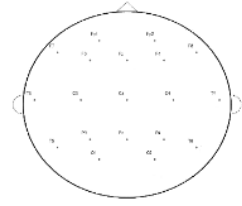

Left frontal-Right frontal (a)

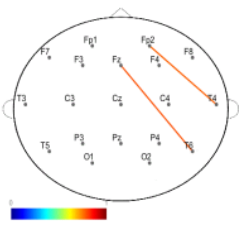

Right frontal-temporal

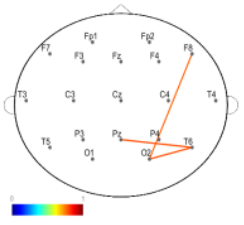

Right temporal-parietal

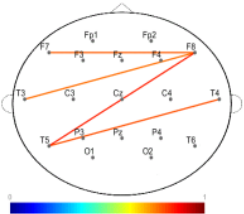

Left temporal-Right temporal (c)

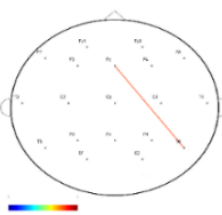

Right frontal-temporal

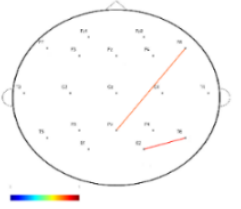

Right temporal-parietal

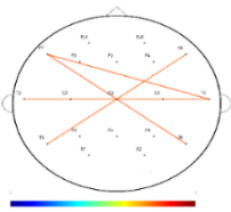

Left temporal-Right temporal

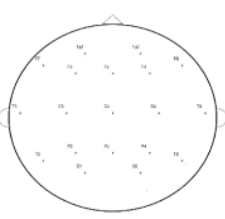

Left frontal-temporal

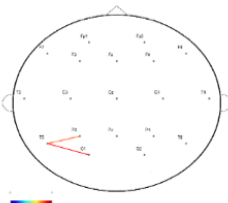

Left temporal-parietal

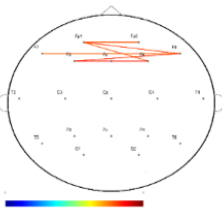

Left frontal-Right frontal

(b)

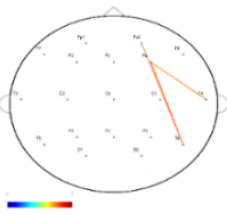

Right frontal-temporal

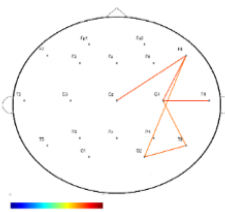

Right temporal-parietal

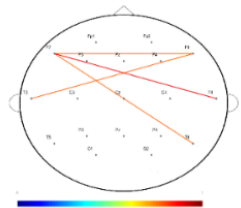

Left temporal-Right temporal

(d)

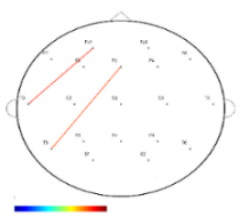

Left frontal-temporal

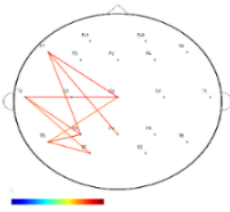

Left temporal-parietal

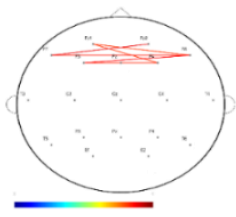

Left frontal-Right frontal

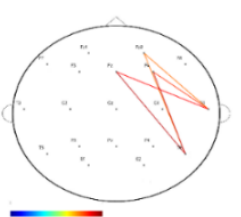

Right frontal-temporal

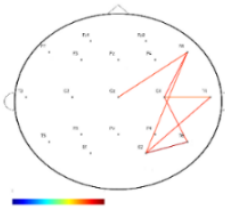

Right temporal-parietal

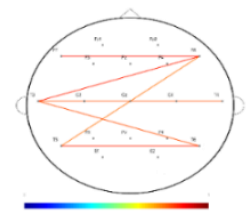

Left temporal-Right temporal

Figure 23. Head map connectivity plot of six local regions yielded from a sample EEG segment of (a) P3 and (b) P4 with epileptic focus in right temporal and (c) P1 and (d) P9 with epileptic focus in left temporal area. Note: Only strong connections are shown. 


\section{Local connections}
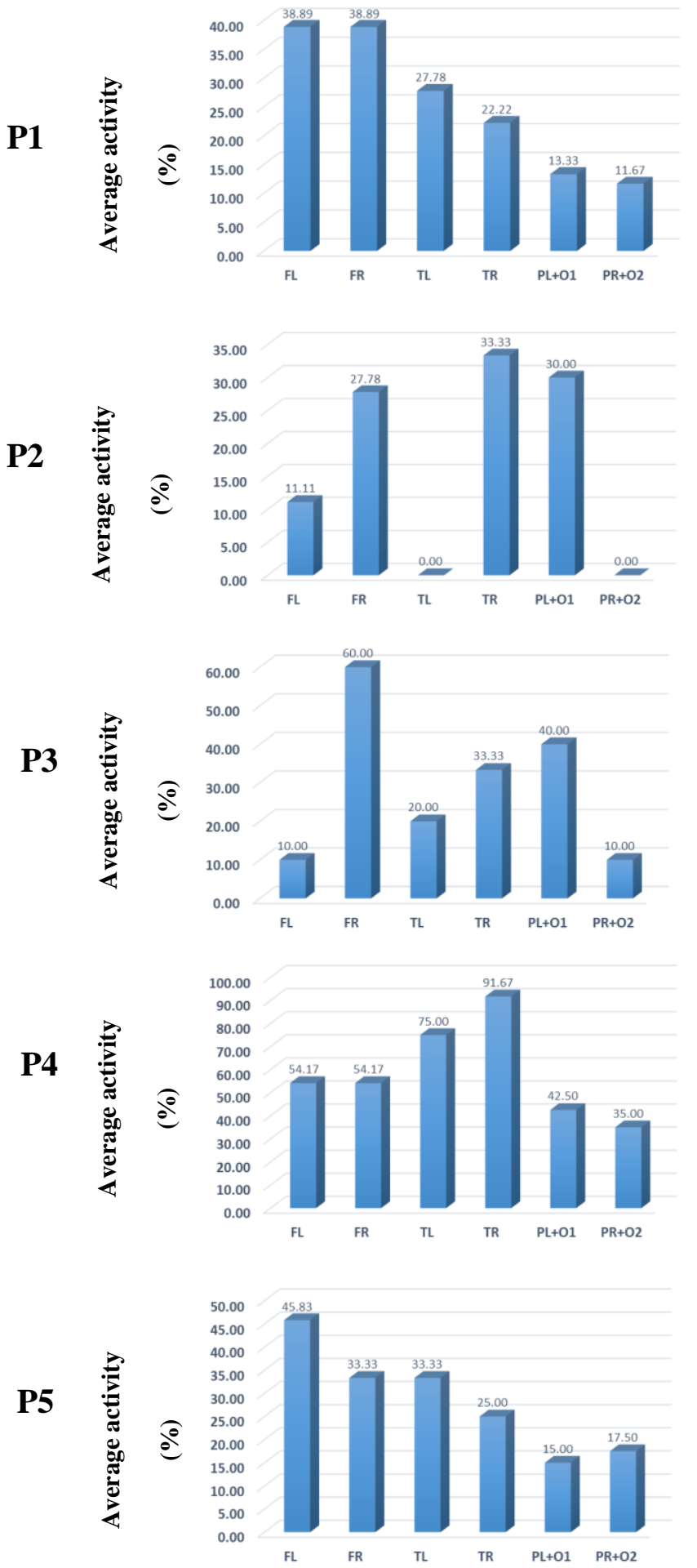

Distant connections
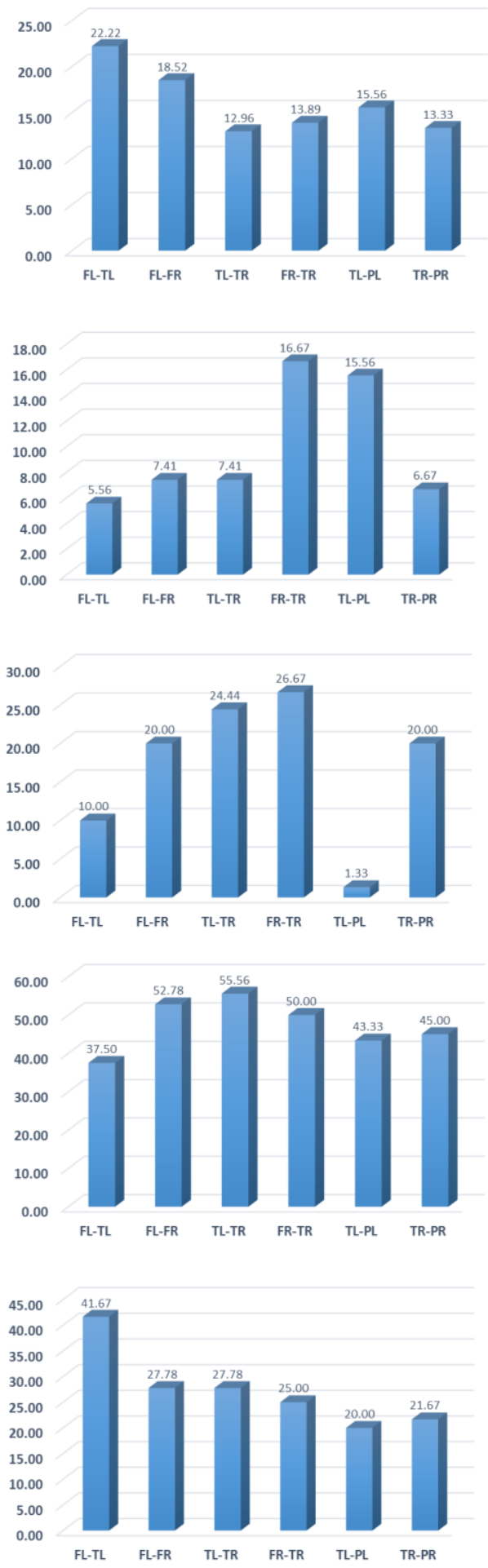

Figure 24 . Average activity percentage of local regions for the 10 patients in the study 
Local connections
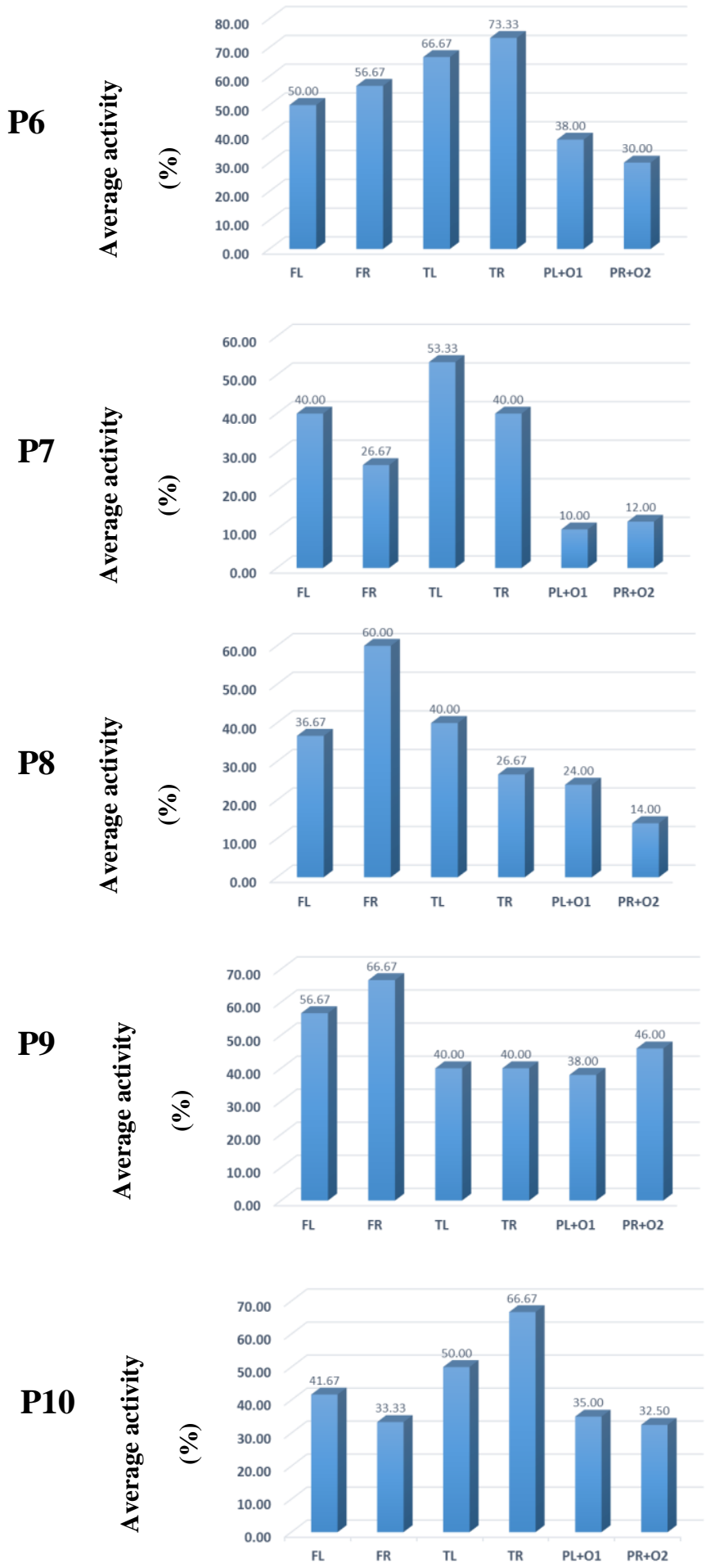

Distant connections
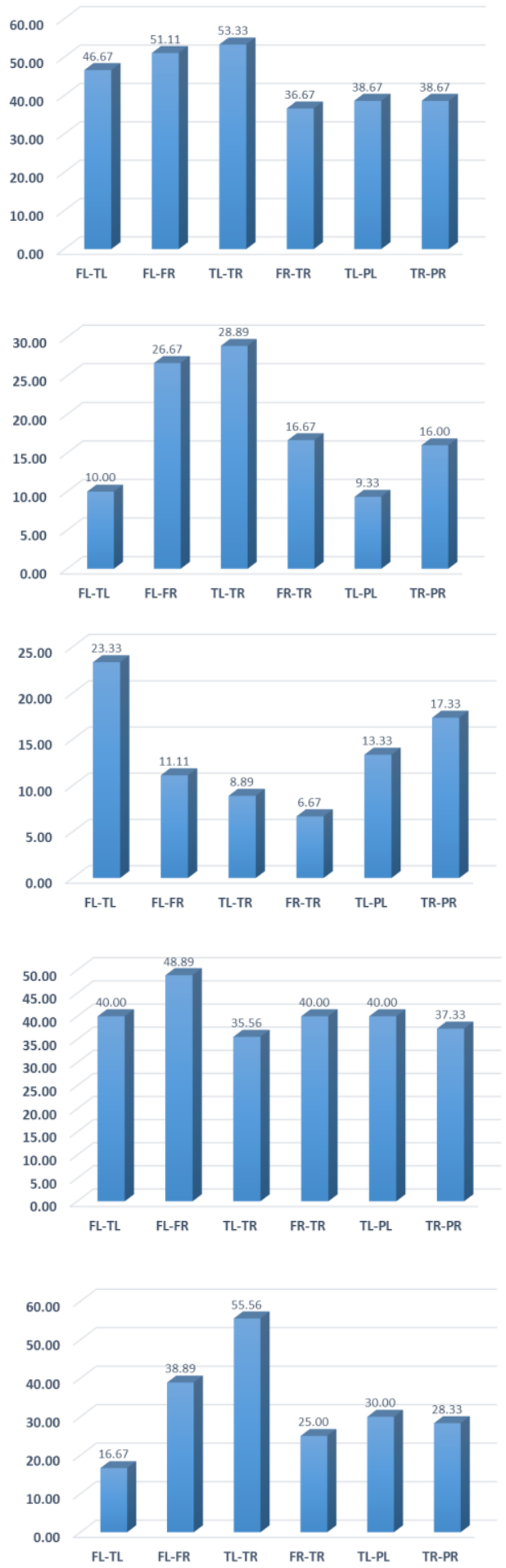

Figure 25. Average activity percentage of local and distant regions for the 10 patients in the study 


\subsection{Conclusion}

In this research endeavor, we have explored the interplay between the epileptogenic zone and the functional connectivity maps resulting from interictal epileptic discharges in focal epilepsy. A collection of 47 segments of interictal scalp EEG containing spikes or sharp waves were extracted. These segments were taken from EEG recordings from ten epileptic patients diagnosed with temporal lobe epilepsy. A nonlinear data-driven method is applied to scalp EEG signals to estimate the phase coupling among brain cortical nodes. Functional connectivity maps were explored both locally and in terms of how the epileptogenic zone can affect distant areas of the brain. Local links were investigated concerning six brain regions (left and right frontal, left and right temporal, left and right parietal and occipital).

Relationships between the local areas were investigated through distant connections. Moreover, obtaining the so-called activity percentages allowed us to assess the regional activities, and most active areas were highlighted for every connectivity map.

The comparison between activities of local areas indicates that the region associated with the epileptic foci results in high coupling in their corresponding connectivity maps. In addition to the affected temporal area, we have observed high synchrony in the frontal zone of the affected hemisphere. Furthermore, we observed strong links connecting the active distant regions resulting in stimulation of temporal and frontal areas of the opposite hemisphere. In general, we conclude that epileptic activity in temporal area irritates both hemispheres in both the temporal and frontal zones. Clearly, the findings reported in this study elicit new understanding of brain activity as relayed in terms of functional activity 
maps in the context of the epileptogenic zone. Consequently, such findings enhance our perception of the disease type in the early stages of the diagnosis and inform medical doctors and clinicians on the most effective steps to consider in presurgical planning. 


\section{Chapter 8}

\section{CONCLUSION AND RECOMMENDATIONS FOR FUTURE WORK}

\subsection{Conclusion}

In this dissertation, the functional connectivity patterns of interictal epileptic data are investigated. The in-between seizure scalp EEG data with 19 electrodes of the 10-20 system, representing various brain modules, has been used in pediatric and adult populations. The nonlinear interdependence of the brain neuronal assemblies was expressed as phase synchrony among neural electrical activities. A nonlinear data-driven technique was applied to the signals in the time domain to estimate the phase coupling among EEG electrodes. The applied method, which is based on the notion of recurrence in chaotic systems, estimates the phase synchronization attributes from a broadband signal without any conversion to the frequency domain. The pairwise phase coupling measures of EEG electrodes were represented in connectivity matrices. The connectivity matrices were investigated by various methods based on the application.

In terms of newly developed methods along with the empirical evaluations that accompany them, in chapter 4 we showed how the interictal EEG recordings of the pediatric population were delineated using the functional connectivity features. A population of 14 pediatric individuals including 7 epileptic patients and 7 normal controls was used. Phase synchrony among brain modules was extracted by utilizing the data-driven recurrence-based method. The implemented algorithm analyzed some higher order attributes of the EEG recordings by the signal reconstruction in the phase space. The connectivity maps of the brain, for a 
given EEG segment, perfectly addressed the phase synchronization between electrodes, as connected electrodes are found to be coupled in the phase domain. The achieved results strongly support the hypothesis of highly connected brain regions in epileptic patients, since connectivity maps of epileptic patients were showing stronger connections in comparison to the control group. The results obtained showed significant differences among connectivity matrices between the two groups $(P-$ value $<0.01)$. The unsupervised k-means clustering algorithm was employed to the feature set for a connectivity-based classification. The applied algorithm benefited from the simplified classification process since there was no training stage. The classification results were obtained with a high accuracy of $92.8 \%$ and with a sensitivity of $85.7 \%$ and a specificity of $100 \%$. The proposed algorithm is a fully automated EEG-based technique that can assist neurologists for the diagnosis of epilepsy.

In chapter 5, the functional connectivity maps of three different interictal epileptic activities characterizing focal and generalized epilepsy were assessed. The interictal epileptic activities including single spike, complex spike, and repetitive spike were utilized for the evaluation of the cortical phase coupling patterns. A model-free data-driven technique was applied to the thirty scalp EEG segments in accordance with the 10-20 system. The strength and number of connections were assessed in four major brain regions (Anterior, Posterior, Left hemisphere and Right hemisphere) to check the localization or globalization of strong connections. The investigation resulted in significant differences $(P-$ value $\ll 0.001)$ between single spike connectivity patterns versus complex and repetitive spikes. These findings come in support of the hypothesis that higher global synchronization are found in 
relation to generalized seizure as compared to the more local couplings that are observed in the case of focal epilepsy. The observed differences between local and global connectivity patterns were supported by performing the analysis of variance among average connectivity matrices of the three groups. These results indicated that, the assessment of the broadband phase synchrony of the brain modules in the form of functional connectivity maps, obtained from scalp EEG, can benefit the epilepsy diagnosis process. This applied technique can help the recognition of focal or generalized epilepsy by extracting the higher order features of the epileptic biomarkers and as a consequence, the quality of epilepsy diagnosis solely based on interictal EEG analysis can be enhanced.

In chapter 6, we have investigated the patterns of interictal activities such as sharp waves, spike, spike-wave complexes and polyspike-wave complexes in focal and generalized epilepsy. The connectivity maps of 43 segments including interictal epileptic activity from four individuals (two with focal and two with generalized epilepsy) were obtained and compared. The significance of the connectivity matrices was assured with the help of surrogate data testing methods. The resulting connectivity maps indicated a higher synchronization due to the presence of interictal epileptiform activity. The number of connections, along with connection strength within and between the four main brain regions, was considered to quantify patterns related to focal and generalized epilepsy. The phase synchrony activities in these areas resulted in different connectivity patterns as a consequence of the various epileptic discharges. The ANOVA test proved the significant difference $(P-$ value $\cong 0)$ among connectivity patterns resulting from focal epilepsy (the spike group) as compared to the patterns of generalized epilepsy (complex and repetitive 
group. In general, we can conclude that the connectivity patterns (the connection strength and propagation patterns) were in direct relation to the type of seizure. These results can help to improve our understanding of the mechanism of the different seizure types. This research can be used to enhance the automatic diagnosis of the different types of epilepsy by randomly selecting EEG segments. A more complete inspection of epileptic discharge connectivity patterns could expose new ways to enhance the location of a seizure focus.

As per our investigation (Rajaei et al., 2016), interictal epileptic activity resulted in high synchronization through the brain and consequently a higher number of strong connections in the resulting brain connectivity maps. We have investigated the differences between connectivity maps in focal and generalized epilepsy, yielding well-defined regional activities in focal epilepsy rather than the activity scattered in all regions of the brain as observed for the generalized case (Rajaei et al., 2017).

Based on these findings, in chapter 7, we have studied the footprints of the epileptogenic zone in the functional connectivity maps of interictal epileptic discharges for focal epilepsy. We have considered 47 segments of scalp EEG including spikes and sharp waves that were recorded from a population of ten individuals diagnosed with temporal lobe epilepsy. The functional cortical relations in terms of phase synchrony was implemented by using a nonlinear method. The resulted functional connectivity maps were assessed by quantifying locally and distant activations areas related to the epileptogenic zone. Six brain functional regions (left and right frontal, left and right temporal, left and right parietal and occipital) were considered to study the interconnections regionally. 
The interactions among the local areas were explored through distant connections. The activity of local regions was evaluated by the average measure, which helped us to highlight the most active areas for every connectivity map.

The evaluation of the activity of local areas showed high synchronization among the electrodes associated with the epileptic focus. In addition to the affected temporal area, we have observed that the activation was spanned to other areas like the frontal zone of the affected hemisphere and the frontal and temporal region of the contralateral hemisphere. Furthermore, we observed strong links connecting the active distant regions. In general, we can conclude that epileptic discharges in temporal areas can affect both hemispheres in the temporal and frontal regions. These findings can elucidate new insights of epileptic brain activity in the context of the epileptogenic zone location.

\subsection{Development and future work recommendation}

Despite many advances in neuroscience, the functional and effective connectivity have a promising long way to be considered as a day to day clinical tool in hospital settings. New advances in data acquisition and storage capabilities, along with the growing hardware and software computing techniques, will pave the way in using the functional connectivity maps in a new and more profound manner. High volume cloud storage facilities are helping in the creation of big data repository, which is a necessary stage for applying the emerging

machine learning tools such as deep learning algorithms. Functional and adaptive connectivity measurements should adapt to these new machine learning techniques, and future algorithms can be implemented along with the modern network science. 
The integration of EEG functional/effective connectivity features with other modalities such as fMRI will link the brain structure with a functional dynamic obtained from the neural electrophysiological activity. 


\section{REFERENCES}

1. Acharya UR, Sree SV, Swapna G, Martis RJ, Suri JS. Automated EEG analysis of epilepsy: a review. Knowledge-Based Syst. 2013;45:147-165.

2. Acharya UR, Sree SV, Chattopadhyay S, Yu W, Ang PCA. APPLICATION OF RECURRENCE QUANTIFICATION ANALYSIS FOR THE AUTOMATED IDENTIFICATION OF EPILEPTIC EEG SIGNALS. International Journal of Neural Systems 2011;21:199-211.

3. Adeli H, Ghosh-Dastidar S, Dadmehr N. A wavelet-chaos methodology for analysis of EEGs and EEG subbands to detect seizure and epilepsy. Biomedical Engineering, IEEE Transactions on 2007;54:205-211.

4. Amini L, Jutten C, Achard S, David O, Soltanian-Zadeh H, Hossein-Zadeh GA, et al. Directed Differential Connectivity Graph of Interictal Epileptiform Discharges. TBME 2011;58:884-893.

5. Bartolomei F, Trébuchon A, Bonini F, Lambert I, Gavaret M, Woodman M, et al. What is the concordance between the seizure onset zone and the irritative zone? A SEEG quantified study. Clinical Neurophysiology 2016;127(2):1157-1162.

6. Bastos AM, Schoffelen JM. A tutorial review of functional connectivity analysis methods and their interpretational pitfalls. Frontiers in Systems Neuroscience 2016;9.

7. Bettus G, Wendling F, Guye M, Valton L, Régis J, Chauvel P, et al. Enhanced EEG functional connectivity in mesial temporal lobe epilepsy. Epilepsy Res. 2008;81:58-68.

8. Bounova G. Octave Networks Toolbox First Release. 2014.

9. Centers for Disease Control and Prevention, (CDC). Epilepsy in adults and access to care--United States, 2010. MMWR Morb.Mortal.Wkly.Rep. 2012;61:909-913.

10. Cheung BLP, Nowak R, Hyong Chol Lee, Drongelen W, Veen BD. Cross Validation for Selection of Cortical Interaction Models From Scalp EEG or MEG. TBME 2012;59:504-514.

11. Coito A, Michel CM, van Mierlo P, Vulliemoz S, Plomp G. Directed Functional Brain Connectivity Based on EEG Source Imaging: Methodology and Application to Temporal Lobe Epilepsy. TBME 2016;63:2619-2628. 
12. Coito A, Plomp G, Genetti M, Abela E, Wiest R, Seeck M, et al. Dynamic directed interictal connectivity in left and right temporal lobe epilepsy. Epilepsia 2015;56:207-217.

13. Delorme A, Makeig S. EEGLAB: an open source toolbox for analysis of singletrial EEG dynamics including independent component analysis. J.Neurosci.Methods 2004;134:9-21.

14. Fisher RS, Boas WvE, Blume W, Elger C, Genton P, Lee P, et al. Epileptic seizures and epilepsy: definitions proposed by the International League Against Epilepsy (ILAE) and the International Bureau for Epilepsy (IBE). Epilepsia 2005;46:470472 .

15. Friston KJ. Functional and Effective Connectivity: A Review. Brain Connectivity 2011;1:13-36.

16. Gautama T, Mandic DP, Van Hulle MM. Indications of nonlinear structures in brain electrical activity. Physical Review E 2003;67:046204.

17. Golshan HM, Hebb AO, Hanrahan SJ, Nedrud J, Mahoor MH. An FFT-based Synchronization Approach to Recognize Human Behaviors using STN-LFP Signal. arXiv preprint arXiv:1612.087802016.

18. Guevara R, Velazquez JLP, Nenadovic V, Wennberg R, Senjanović G, Dominguez LG. Phase synchronization measurements using electroencephalographic recordings. Neuroinformatics 2005;3:301-313.

19. Hosseini SAH, Sohrabpour A, He B. Electromagnetic source imaging using simultaneous scalp EEG and intracranial EEG: An emerging tool for interacting with pathological brain networks. Clinical Neurophysiology 2018;129:168-187.

20. Junfeng Sun, Xiangfei Hong, Shanbao Tong. Phase Synchronization Analysis of EEG Signals: An Evaluation Based on Surrogate Tests. TBME 2012;59:22542263.

21. Kalitzin SN, Parra J, Velis DN, da Silva FHL. Quantification of Unidirectional Nonlinear Associations Between Multidimensional Signals. TBME 2007;54:454461.

22. Kurths J, Romano MC, Thiel M, Osipov GV, Ivanchenko MV, Kiss IZ, et al. Synchronization analysis of coupled noncoherent oscillators. Nonlinear Dyn. 2006;44:135-149.

23. Lakshmanan M, Senthilkumar DV. Transition from Phase to Generalized Synchronization. Dynamics of Nonlinear Time-Delay Systems: Springer, Berlin, Heidelberg; 2011: 201-226. 
24. Lehnertz K, Bialonski S, Horstmann M, Krug D, Rothkegel A, Staniek M, et al. Synchronization phenomena in human epileptic brain networks. J.Neurosci.Methods 2009;183:42-48.

25. Majumdar K. An FFT based measure of phase synchronization. arXiv preprint qbio/0612004 2006.

26. Marwan N, Romano MC, Thiel M, Kurths J. Recurrence plots for the analysis of complex systems. Physics reports 2007;438:237-329.

27. Marwan N, Wessel N, Meyerfeldt U, Schirdewan A, Kurths J. Recurrence-plotbased measures of complexity and their application to heart-rate-variability data. Physical review E 2002;66:026702.

28. Meesters S, Ossenblok P, Colon A, Wagner L, Schijns O, Boon P, et al. Modeling of intracerebral interictal epileptic discharges: Evidence for network interactions. Clinical Neurophysiology 2018;129(6):1276-1290.

29. Mégevand P, Seeck M. Electroencephalography, magnetoencephalography, and source localization: their value in epilepsy. Current opinion in neurology 2018;31:176.

30. Mégevand P, Spinelli L, Genetti M, Brodbeck V, Momjian S, Schaller K, et al. Electric source imaging of interictal activity accurately localises the seizure onset zone. Journal of neurology, neurosurgery, and psychiatry 2014;85:38-43.

31. Mercedes C, Adjouadi M, Ayala M, Tito M. Pattern extraction in interictal EEG recordings towards detection of electrodes leading to seizures. Biomed.Sci.Instrum. 2005;42:243-248.

32. Natarajan K, Acharya R, Alias F, Tiboleng T, Puthusserypady SK. Nonlinear analysis of EEG signals at different mental states. BioMedical Engineering OnLine 2004;3:7.

33. National Institute of Neurological Disorders and Stroke (US). Office of Communications and Public Liaison. The Epilepsies and Seizures: Hope Through Research. : Department of Health and Human Services, NIH, National Institute of Neurological Disorders and Stroke, 2015.

34. Noachtar S, Binnie C, Ebersole J, Mauguière F, Sakamoto A, Westmoreland B. A glossary of terms most commonly used by clinical electroencephalographers and proposal for the report form for the EEG findings. The International Federation of Clinical Neurophysiology. Electroencephalogr Clin Neurophysiol Suppl $1999 ; 52: 21-41$. 
35. Peng Xu, Xiuchun Xiong, Qing Xue, Peiyang Li, Rui Zhang, Zhenyu Wang, et al. Differentiating Between Psychogenic Nonepileptic Seizures and Epilepsy Based on Common Spatial Pattern of Weighted EEG Resting Networks. TBME 2014;61:1747-1755.

36. Pohlmann-Eden B, Beghi E, Camfield C, Camfield P. The first seizure and its management in adults and children. BMJ 2006;332:339-342.

37. Ponten SC, Douw L, Bartolomei F, Reijneveld JC, Stam CJ. Indications for network regularization during absence seizures: weighted and unweighted graph theoretical analyses. Exp.Neurol. 2009;217:197-204.

38. Puthanmadam Subramaniyam N. Recurrence network analysis of EEG signals. 2016.

39. Rajaei H, Cabrerizo M, Janwattanapong P, Pinzon-Ardila A, Gonzalez-Arias S, Adjouadi M. Connectivity Maps of Different Types of Epileptogenic Patterns. 2016:1019,1020,1021.

40. Rajaei H, Cabrerizo M, Janwattanapong P, Pinzon A, Gonzalez-Arias S, Barreto A, et al. Connectivity Dynamics of Interictal Epileptiform Activity. 2017:425-430.

41. Rajaei H, Cabrerizo M, Sargolzaei S, Pinzon-Ardila A, Gonzalez-Arias S, Adjouadi M. Pediatric epilepsy: Clustering by functional connectivity using phase synchronization. 2015:1-4.

42. Rangaprakash D. Connectivity analysis of multichannel EEG signals using recurrence based phase synchronization technique. Comput.Biol.Med. 2014;46:1121.

43. Rangaprakash D, Hu X, Deshpande G. Phase synchronization in brain networks derived from correlation between probabilities of recurrences in functional MRI data. Int.J.Neural Syst. 2013;23:1350003.

44. Rangaprakash D, Pradhan N. Study of phase synchronization in multichannel seizure EEG using nonlinear recurrence measure. Biomedical Signal Processing and Control 2014;11:114-122.

45. Sadock BJ, Sadock VA, Ruiz P. Kaplan and Sadock's synopsis of psychiatry. 11. ed., internat. ed. ed. Philadelphia [u.a.]: Wolters Kluwer, 2015.

46. Sakkalis V. Review of advanced techniques for the estimation of brain connectivity measured with EEG/MEG. Comput.Biol.Med. 2011;41:1110-1117. 
47. Sargolzaei S, Cabrerizo M, Goryawala M, Eddin AS, Adjouadi M. Functional connectivity network based on graph analysis of scalp EEG for epileptic classification. 2013:1-4.

48. Schelter B, Winterhalder M, Timmer J. Handbook of time series analysis: recent theoretical developments and applications. : John Wiley and Sons, 2006.

49. Schreiber T, Schmitz A. Surrogate time series. Physica D 2000;142:346-382.

50. Shafi MM, Westover MB, Oberman L, Cash SS, Pascual-Leone A. Modulation of EEG functional connectivity networks in subjects undergoing repetitive transcranial magnetic stimulation. Brain Topogr. 2014;27:172-191.

51. Smith SJ. EEG in the diagnosis, classification, and management of patients with epilepsy. J.Neurol.Neurosurg.Psychiatry. 2005;76 Suppl 2:7.

52. Sobayo T, Fine AS, Gunnar E, Kazlauskas C, Nicholls D, Mogul DJ. Synchrony Dynamics Across Brain Structures in Limbic Epilepsy Vary Between Initiation and Termination Phases of Seizures. TBME 2013;60:821-829.

53. Sohrabpour A, Shuai Ye, Worrell GA, Wenbo Zhang, Bin He. Noninvasive Electromagnetic Source Imaging and Granger Causality Analysis: An Electrophysiological Connectome (eConnectome) Approach. TBME 2016;63:2474-2487.

54. Staljanssens W, Strobbe G, Van Holen R, Keereman V, Gadeyne S, Carrette E, et al. EEG source connectivity to localize the seizure onset zone in patients with drug resistant epilepsy. NeuroImage: Clinical 2017;16:689-698.

55. Stam CJ. Modern network science of neurological disorders. Nature Reviews Neuroscience 2014;15:683-695.

56. Stam CJ, Nolte G, Daffertshofer A. Phase lag index: Assessment of functional connectivity from multi channel EEG and MEG with diminished bias from common sources. Hum.Brain Mapp. 2007;28:1178-1193.

57. Stam CJ, Reijneveld JC. Graph theoretical analysis of complex networks in the brain. Nonlinear biomedical physics 2007;1:3.

58. Sun J, Hong X, Tong S. Phase synchronization analysis of EEG signals: an evaluation based on surrogate tests. IEEE Transactions on Biomedical Engineering 2012;59:2254-2263.

59. Tatum WO. Handbook of EEG interpretation. 2. ed. ed. New York: DemosMedical, 2014. 
60. Taylor PN, Goodfellow M, Wang Y, Baier G. Towards a large-scale model of patient-specific epileptic spike-wave discharges. Biol.Cybern. 2013;107:83-94.

61. van Diessen E, Otte WM, Stam CJ, Braun KP, Jansen FE. Electroencephalography based functional networks in newly diagnosed childhood epilepsies. Clinical Neurophysiology 2016;127:2325-2332.

62. Verhoeven T, Coito A, van Mierlo P, Seeck M, Michel C, Plomp G, et al. Using Random Forest for Diagnosis and Lateralization of Temporal Lobe Epilepsy from EEG-based Directed Functional Connectivity. 2016;57:64.

63. West BJ. Fractal Physiology and Chaos in Medicine (Studies of Nonlinear Phenomena in Life Science, Vol 1). 1991.

64. Wilke C, Lei Ding, Bin He. Estimation of Time-Varying Connectivity Patterns Through the Use of an Adaptive Directed Transfer Function. TBME 2008;55:25572564.

65. Wilke C, Worrell G, He B. Graph analysis of epileptogenic networks in human partial epilepsy. Epilepsia 2011;52:84-93.

66. Yifan Zhao, Billings SA, Hua-Liang Wei, Sarrigiannis PG. A Parametric Method to Measure Time-Varying Linear and Nonlinear Causality With Applications to EEG Data. TBME 2013;60:3141-3148.

67. Zbilut JP, Webber CL. Recurrence quantification analysis. Wiley encyclopedia of biomedical engineering 2006. 
VITA

HODA RAJAEI

2000-2005

B.S., Electrical and Electronic Engineering

Shahid Bahonar University of Kerman

Kerman, Iran

2005-2013

Instrumentation and Control Engineer

FSIC and PIDEC Companies

Shiraz, Iran

2010-2012

M.S., Instrumentation and Automation Engineering

Shiraz University

Shiraz, Iran

2014-2018

Ph.D. Candidate, Electrical Engineering

Florida International University

Miami, Florida

Graduate School Dissertation Year Fellowship (DYF), Florida International University (Spring 2018)

Third place in the oral presentation in FIU graduate appreciation week, Florida International University (March 2017)

Startup FIU fellowship, Florida International University, (December 2016)

IEEE EMBC2016 NSF young professional award, Georgia Institute of Technology, (Aguste 2016)

Second place in oral presentation FIU graduate appreciation week, Florida International University (April 2016)

Second place in poster presentation FIU graduate appreciation week, Florida International University (April 2016) First Place in engineering group for graduate student appreciation week (April 2015)

\section{PUBLICATIONS AND PRESENTATIONS}

Rajaei H., Cabrerizo M., Janwattanapong,P., Pinzon A., Gonzalez-Arias S., Barreto A., Andrian, J., Rishe, N., and Adjouadi M., "Dynamics and Distant Effects of Temporal 
Epileptogenic Focus Using Functional Connectivity Maps", IEEE Transactions on Biomedical Engineering, under review

Janwattanapong,P., Cabrerizo, M., Rajaei, H., Fang, C., Barreto, A., Andrian, J., Pinzon, A., Gonzales-Arias, S., Rishe, N. and Adjouadi, A., "Analysis of Brain Connectivity Patterns in Epilepsy Using Effective Information Flow and Generalized Partial Directed Coherence", Biomedical Signal Processing and Control, under review

Rajaei H., Cabrerizo M., Janwattanapong,P., Pinzon A., Gonzalez-Arias S., Barreto A., and Adjouadi M., "Connectivity Dynamics of Interictal Epileptiform Activity", IEEE BIBE2017 conference, pp. 425 - 430, Washington DC, October 23-25, 2017.

Janwattanapong P., Cabrerizo M., Fang C., Rajaei H., Pinzon A., Gonzalez-Arias S., and Adjouadi M., "Classification of Interictal Epileptiform Discharges Using Partial Directed Coherence", IEEE BIBE2017 conference, pp. 473 - 478, Washington DC, October 23-25, 2017.

Mafi M., Rajaei H., Cabrerizo M., and Adjouadi M., "A Robust Edge Detection Approach in the Presence of High Impulse Noise Intensity Through Switching Adaptive Median and Fixed Weighted Mean Filtering", IEEE Transaction on Image Processing, Vol. 27(11), pp. $5475-5490,2018$.

Rajaei H., Cabrerizo M., Janwattanapong P., Pinzon A., Gonzalez-Arias S., Barreto A., Andrian J., Rishe N. and Adjouadi M., "Interictal EEG Analysis Based on Phase Synchronization”, Biotechnology and Bioinformatic Symposium (BIOT2016), December 2016, Provo Utah

Janwattanapong,P., Cabrerizo, M., Rajaei, H., Pinzon-Ardila, A., Gonzalez-Arias,S., and Adjouadi, M., "Epileptogenic Brain Connectivity Patterns Using Scalp EEG”, GlobalSIP 2016 Conference, Decembre 2016, Washington DC

Rajaei, H., Cabrerizo, M., Janwattanapong,P., Pinzon-Ardila, A., Gonzalez-Arias,S., and Adjouadi, M., "Connectivity Maps Of Different Types of Epileptogenic Patterns", IEEE EMBC 2016 Conference, pp. 1018 - 1021, DOI: 10.1109/EMBC.2016.7590875, Orlando, FL, Aug. 17-20, 2016.

Rajaei, H., Cabrerizo, M., Sargolzaei, S., Pinzon-Ardila, A., Gonzalez-Arias,S., and Adjouadi, M., "Pediatric Epilepsy: Clustering by Functional Connectivity Using Phase Synchronization”, IEEE BIOCAS 2015 Conference, DOI: 10.1109/BioCAS.2015.7348368, pp. 1-4, 2015. Oct. 2015, Atlanta, Georgia

Sargolzaei, S., Cabrerizo, M., Sargolzaei, A., Noei, S., Eddin, A. S., Rajaei, H., and Adjouadi, M. (2015). "A Probabilistic Approach for Pediatric Epilepsy Diagnosis Using Brain Functional Connectivity Networks". Journal of BMC Bioinformatics, Vol. 16, Suppl. 7, DOI: 10.1186/1471-2105-16-S7-S9, PMID: 25953124, April 2015. 